\title{
Quantum deformations of the one-dimensional Hubbard model
}

\author{
Niklas Beisert $^{1}$ and Peter Koroteev ${ }^{1,2,3}$ \\ ${ }^{1}$ Max-Planck-Institut für Gravitationsphysik, Albert-Einstein-Institut, Am Mühlenberg 1, \\ 14476 Potsdam, Germany \\ 2 Institute for Theoretical and Experimental Physics, B. Cheremushkinskaya, \\ 25, Moscow 117259, Russia \\ ${ }^{3}$ Institute for Nuclear Research, Prospekt 60-letiya Oktyabrya 7a, Moscow 117312, Russia \\ E-mail: nbeisert@aei.mpg.de and koroteev@aei.mpg.de
}

Received 3 April 2008

Published 28 May 2008

Online at stacks.iop.org/JPhysA/41/255204

\begin{abstract}
The centrally extended superalgebra $\mathfrak{p s u}(2 \mid 2) \ltimes \mathbb{R}^{3}$ was shown to play an important role for the integrable structures of the one-dimensional Hubbard model and of the planar AdS/CFT correspondence. Here we consider its quantum deformation $\mathrm{U}_{q}\left(\mathfrak{p} \mathfrak{s u}(2 \mid 2) \ltimes \mathbb{R}^{3}\right)$ and derive the fundamental $R$-matrix. From the latter we deduce an integrable spin-chain Hamiltonian with three independent parameters and the corresponding Bethe equations to describe the spectrum on periodic chains. We relate our Hamiltonian to a two-parametric Hamiltonian proposed by Alcaraz and Bariev which can be considered a quantum deformation of the one-dimensional Hubbard model.
\end{abstract}

PACS numbers: $02.30 . I k, 02.20 . U w, 75.10 . P q$

\section{Introduction and overview}

Finding the spectrum of a quantum mechanical model is an intricate problem. Indeed, for generic models there is no complete analytic solution to the spectrum essentially because nonlinear interaction terms in the Hamiltonian easily make the problem chaotic and intractable. Only very few models, such as the harmonic oscillator, are solvable exactly. Somewhere in between these two extremes live the integrable models. They may contain highly nontrivial interactions, but they can nevertheless be solved completely by the right ansatz for the wavefunctions. Such a wavefunction will depend on a couple of parameters, and quantization conditions will impose a system of equations on them. There need not be a general analytic solution to these equations-after all the spectrum of integrable models is usually highly non-trivial-nevertheless the reduction to a small number of parameters is sufficient to make the spectral problem much more tractable than for generic quantum mechanical models. 
Typical integrable models are formulated in $(1+1)$ or two dimensions. They include field theories, nonlinear sigma models, particle models, vertex models and spin chains (we shall focus on the latter in this paper). A plethora of integrable models with all kinds of features is known to date, and it appears near impossible to make a complete census. A central insight toward this goal was made in the 1980s by the Leningrad/St. Petersburg School who related integrability to the existence of large hidden symmetries. Through the enumeration of suitable symmetry algebras one can hope to classify the integrable models.

For example, a very large class of integrable spin chains can be derived and investigated using Yangians and quantum affine algebras $\mathrm{U}_{q}(\hat{\mathfrak{g}})$ [1]. In particular, the Heisenberg XXX (algebraic) spin chain and its relatives with different symmetry algebra $\mathfrak{g}$ and/or different representation are all based on the Yangian double of $\mathfrak{g}$. Likewise, the quantum-deformed XXZ-like (trigonometric) spin-chain cousins are related to the quantum affine algebra $\mathrm{U}_{q}(\hat{\mathfrak{g}})$. XYZ-like (elliptic) spin chains also have a similar but much more elaborate underlying symmetry algebra.

A famous integrable spin-chain model that has escaped this classification for a long time is the one-dimensional Hubbard model, see [2]. It is a model of electrons propagating on a chain of nuclei. Each nucleus site can either be unoccupied, singly occupied with electron spin pointing up/down or doubly occupied with opposing electron spins. In total there are four states per site ( $c_{\alpha}^{\dagger}$ is a fermionic electron creation operator)

$$
|\circ\rangle, \quad|\uparrow\rangle \sim c_{1}^{\dagger}|\circ\rangle, \quad|\downarrow\rangle \sim c_{2}^{\dagger}|\circ\rangle, \quad|\uparrow\rangle \sim c_{1}^{\dagger} c_{2}^{\dagger}|\circ\rangle .
$$

The middle two states are considered fermionic while the outer two states are overall bosonic. This model is exciting because it shows some characteristics of superconductivity, and therefore it is very desirable to understand its foundations well. Integrability was established by Lieb and Wu who also solved the spectrum by means of the Bethe ansatz [3]. An $R$-matrix which encodes the integrable structure was later found by Shastry [4]. On the one hand, the $R$-matrix is the foundation for much of the integrable machinery, such as the algebraic Bethe ansatz [5,6]. On the other hand, this particular $R$-matrix is rather exceptional because unlike most other known $R$-matrices it cannot be written as a function of the difference of two spectral parameters. Altogether, the algebraic origin of the $R$-matrix remained mysterious. It is well known that it is symmetric under two undeformed $\mathfrak{s u}(2)$ algebras: spin and (twisted) eta-pairing [7] symmetry. Therefore one may expect the underlying algebra to be of Yangian (algebraic) rather than of the quantum affine (trigonometric) type. Indeed, two $\mathfrak{s u}(2)$ Yangian algebras were identified [8], but they are not sufficient to explain the $R$-matrix. A fusion procedure of two $X X$ models was used to derive the $R$-matrix and explain its features, but it seems very specialized to the model at hand and it hardly illuminates the symmetries.

New insight into the algebraic structure came from a very different and unexpected direction: the field of gauge theory, string theory and the so-called AdS/CFT correspondence, which relate certain pairs of gauge and string theories. In that context it was observed that $\mathcal{N}=4$ superconformal Yang-Mills theory in the 't Hooft limit and its dual, IIB string theory on the $\mathrm{AdS}_{5} \times S^{5}$, both display signs of integrability [9], see [10,11] for reviews and further references. The asymptotic coordinate Bethe ansatz [12] for the gauge theory spin chain leads to (two copies of) an interesting scattering matrix [13] which is not of difference form. A construction for strings in light-cone gauge [14] leads to an equivalent $S$-matrix [15]. The scattering particles have four flavors $\left|\phi^{1}\right\rangle,\left|\phi^{2}\right\rangle,\left|\psi^{1}\right\rangle,\left|\psi^{2}\right\rangle$, the former two being bosonic and the latter two being fermionic. The set of particle flavors is equivalent to the states of a site in the Hubbard model

$$
|0\rangle \sim\left|\phi^{1}\right\rangle, \quad|\uparrow\rangle \sim\left|\psi^{1}\right\rangle, \quad|\downarrow\rangle \sim\left|\psi^{2}\right\rangle, \quad|\uparrow\rangle \sim\left|\phi^{2}\right\rangle,
$$


and it was observed that the $S$-matrix has a structure reminiscent of the $R$-matrix for the Hubbard model [16]. Indeed, the two matrices can be mapped into each other exactly [17] which leads to a very curious connection between string theory and the integrable structure of the Hubbard model. This link is also reflected in the asymptotic Bethe equations for planar AdS/CFT [18] which contain (two copies) of the Lieb-Wu equations in disguise.

The large amount of supersymmetry present in the string/gauge theory system, the superalgebra $\mathfrak{p} \mathfrak{s u}(2,2 \mid 4)$, thus made its way into the integrable structure of the one-dimensional Hubbard model: what remains of this symmetry in the above scattering picture is (two copies of) $\mathfrak{s u}(2 \mid 2)$ [10]. A crucial point for the further understanding was that the symmetry is centrally extended by gauge transformations inherent to the gauge theory [13] or by residual transformations in the light-cone gauge for string theory [19]. The symmetry of the $S$-matrix turns out to be an (exceptional) three-fold central extension $\mathfrak{h}$ of the $\mathfrak{p s u}(2 \mid 2)$ superalgebra ${ }^{4}$

$$
\mathfrak{h}:=\mathfrak{p s u}(2 \mid 2) \ltimes \mathbb{R}^{3}=\mathfrak{s u}(2 \mid 2) \ltimes \mathbb{R}^{2} .
$$

This algebra contains the two well-known bosonic $\mathfrak{s u ( 2 )}$ symmetries of the Hubbard model which relate the two bosonic and two fermionic states, respectively. However, the additional fermionic generators of the algebra also relate the bosons to the fermions and vice versa. In fact the algebra is strong enough to fully constrain the form of the $R$-matrix [13].

The proper framework for the symmetries of the $R$-matrix and thus for the integrable structure of the one-dimensional Hubbard model is expected to be a quasi-triangular Hopf algebra [20]. The goal is then to find the universal $R$-matrix of which the above $R$-matrix is the fundamental representation. However this requires to first identify the complete symmetry algebra of the $R$-matrix. Generically one may expect the algebra to be a Yangian double: a deformation of the universal enveloping algebra of the loop algebra of the underlying symmetry $\mathfrak{h}$. Indeed many of the Yangian generators have been identified [21, 22]. An investigation of the classical limit [23] of the $R$-matrix has then revealed the complete classical structure in terms of a quasi-triangular bialgebra [24] based on a curious deformation of the loop algebra $\mathfrak{u}(2 \mid 2)\left[u, u^{-1}\right]$. This result shows that in addition to the central charges, there must also be inner automorphisms.

At least two important steps remain to be taken: first, the classical bialgebra needs to be quantized to a Yangian double. Second, the universal $R$-matrix for the Yangian needs to be established which makes the Yangian into a quasi-triangular Hopf algebra. However, it is not easy to deal with Yangian doubles and their algebraic structure because proper quantization of the higher levels is somewhat unintuitive and specialized to the algebra $\mathfrak{g}$. Instead one usually considers the corresponding quantum affine algebra $\mathrm{U}_{q}(\hat{\mathfrak{g}})$ of which the Yangian is a contraction limit for $q \rightarrow 1$. Here one pays the price that the $\mathfrak{g}$ symmetry is quantum deformed and not as manifest as in the Yangian. Instead one gains a uniform treatment for the quantum deformation of the Kac-Moody structure of the whole of the affine algebra $\hat{\mathfrak{g}}$. It is the aim of the present paper to lay the foundations for the quantum deformation of the integrable structure of the one-dimensional Hubbard model. Here we shall start with the quantum deformation $U_{q}(\mathfrak{h})$ of the algebra $\mathfrak{h}$ and leave the full quantum affine algebra $\mathrm{U}_{q}(\hat{\mathfrak{h}})$ (or rather the deformed $\mathrm{U}_{q}\left(\mathfrak{u}(2 \mid 2)\left[u, u^{-1}\right]\right)$ ) and its quasi-triangular structure for future work; the corresponding Yangian double would follow as the limit $q \rightarrow 1$. We will then derive the fundamental $R$-matrix which should be understood as the quantum deformation of Shastry's $R$-matrix for the Hubbard model. We apply it to derive the Bethe equations for periodic wavefunctions and a three-parameter family of Hamiltonians with $\mathrm{U}_{q}(\mathfrak{s u}(2) \times \mathfrak{s u}(2))$ symmetry which includes the Hubbard Hamiltonian as a special case.

4 For simplicity we shall consider the algebra to be complex and do not distinguish between $\mathfrak{p s u}(2 \mid 2), \mathfrak{p s l}(2 \mid 2)$ or $\mathfrak{p s l}(2 \mid 2, \mathbb{C})$. Reality conditions refer to the real version $\mathfrak{p s u}(2 \mid 2)$ of the algebra. 
In fact, many attempts have been made to modify and generalize the Hubbard Hamiltonian due to the exceptional properties of the Hubbard model. Widely discussed modifications are the EKS model [25], the supersymmetric U-model [26] and its quantum deformation [27] as well as the $\mathfrak{s u}(n)$ Hubbard models [28] These can all be explained with the available integrability toolkit: the EKS model is a model based on the fundamental representation of $\mathfrak{u}(2 \mid 2)$ and the supersymmetric U-model is based on the four-dimensional representation of $\mathfrak{s u}(2 \mid 1)$. These models are somewhat similar to the Hubbard model, but they do not include it as a special case. The $\mathfrak{s u}(n)$ Hubbard models employ an external coloring of states which preserves integrability. This coloring can be applied to any integrable model with conserved charges [29] and it does not alter the underlying symmetry. Further similar models have been discussed in [30]. An important class of models which is also discussed in this context consists of the supersymmetric t-J model [31] and some of its deformations such as the Bariev model [32] and others [33]. The main difference is that these models use a three-dimensional representation on each site and thus the Hilbert space is very different from one of the Hubbard models. The only known true deformation of the Hubbard model appears to be a Hamiltonian composed by Alcaraz and Bariev [34]. The Hamiltonian contains substantially more terms and so far it has not been investigated further in the literature. The Bethe equations for this model were given in [34], and the bear some resemblance with those for the XXZ model. Thus it is conceivable that the Alcaraz-Bariev model is a quantum deformation of the Hubbard model. We shall address the question whether we can recover this Hamiltonian at the end of our work.

The present paper is organized as follows: we start with a technical part concerning the algebra, $R$-matrix and Bethe ansatz in sections $2-4$, respectively. In the second part consisting of of section 5 we apply the obtained results to a concrete spin-chain model. It is not necessary to read the earlier sections (in full detail) to understand the later sections.

First the $\mathrm{U}_{q}(\mathfrak{h})$ symmetry is introduced in section 2 . We also sketch finite representations of the algebra with particular focus on the fundamental representation needed for the derivation of the fundamental $R$-matrix in section 3. We then perform the nested Bethe ansatz for this $R$-matrix in section 4 to obtain the Bethe equations for a periodic chain. Finally, in section 5 we derive a class of integrable Hamiltonians associated with the $R$-matrix. These constitute quantum deformations of the Hubbard Hamiltonian. In particular, we recover one of the models proposed by Alcaraz and Bariev. We conclude and give an outlook of open problems in section 6 .

\section{The Hopf algebra $\mathbf{U}_{q}\left(\mathfrak{s u}(2 \mid 2) \ltimes \mathbb{R}^{2}\right)$}

We start with the construction of the symmetry algebra underlying quantum deformations of the one-dimensional Hubbard model. This part is rather technical in nature and can be skipped or be used as a reference for the following sections.

\subsection{From $\mathfrak{s u}(2 \mid 2)$ to $\mathrm{U}\left(\mathfrak{s u}(2 \mid 2) \ltimes \mathbb{R}^{2}\right)$}

We start by introducing the universal enveloping algebra $\mathrm{U}\left(\mathfrak{s u}(2 \mid 2) \ltimes \mathbb{R}^{2}\right)$ step by step starting from the Lie superalgebra $\mathfrak{s u}(2 \mid 2)$. This will help us to understand and derive the quantum deformation $\mathrm{U}_{q}\left(\mathfrak{s u}(2 \mid 2) \ltimes \mathbb{R}^{2}\right)$.

Lie superalgebra. The Lie superalgebra $\mathfrak{s u}(2 \mid 2)$ is generated by the $\mathfrak{s u}(2) \times \mathfrak{s u}(2)$ generators $\mathfrak{R}_{b}^{a}, \mathfrak{L}^{\alpha}{ }_{\beta}$, the supercharges $\mathfrak{Q}_{b}^{\alpha}, \mathfrak{S}^{a}{ }_{\beta}$ and the central charge $\mathfrak{C}^{5}$ The Lie brackets of the $\mathfrak{s u}(2)$

5 To obtain the simple Lie algebra $\mathfrak{p s u}(2 \mid 2)$ from $\mathfrak{s u}(2 \mid 2)$ we would have to project out this central element. 


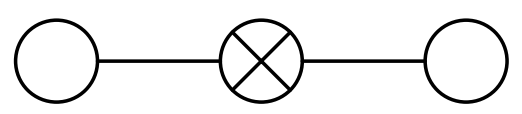

Figure 1. Distinguished Dynkin diagram of $\mathfrak{s u}(2 \mid 2)$.

generators take the standard form

$$
\begin{array}{lll}
{\left[\mathfrak{R}^{a}{ }_{b}, \mathfrak{R}^{c}{ }_{d}\right]=\delta_{b}^{c} \mathfrak{R}^{a}{ }_{d}-\delta_{d}^{a} \mathfrak{R}^{c}{ }_{b},} & {\left[\mathfrak{L}^{\alpha}{ }_{\beta}, \mathfrak{L}^{\gamma}{ }_{\delta}\right]=\delta_{\beta}^{\gamma} \mathfrak{L}^{\alpha}{ }_{\delta}-\delta_{\delta}^{\alpha} \mathfrak{L}_{\beta}{ }_{\beta},} \\
{\left[\mathfrak{R}^{a}{ }_{b}, \mathfrak{Q}^{\gamma}{ }_{d}\right]=-\delta_{d}^{a} \mathfrak{Q}_{b}{ }_{b}+\frac{1}{2} \delta_{b}^{a} \mathfrak{Q}^{\gamma}{ }_{d},} & & {\left[\mathfrak{L}^{\alpha}{ }_{\beta}, \mathfrak{Q}^{\gamma}{ }_{d}\right]=\delta_{\beta}^{\gamma} \mathfrak{Q}^{\alpha}{ }_{d}-\frac{1}{2} \delta_{\beta}^{\alpha} \mathfrak{Q}^{\gamma}{ }_{d},} \\
{\left[\mathfrak{R}^{a}{ }_{b}, \mathfrak{S}^{c}{ }_{\delta}\right]=\delta_{b}^{c} \mathfrak{S}^{a}{ }_{\delta}-\frac{1}{2} \delta_{b}^{a} \mathfrak{S}^{c}{ }_{\delta},} & & {\left[\mathfrak{L}^{\alpha}{ }_{\beta}, \mathfrak{S}^{c}{ }_{\delta}\right]=-\delta_{\delta}^{\alpha} \mathfrak{S}^{c}{ }_{\beta}+\frac{1}{2} \delta_{\beta}^{\alpha} \mathfrak{S}^{c}{ }_{\delta} .}
\end{array}
$$

The Lie brackets of two supercharges yield

$$
\left\{\mathfrak{Q}_{b}^{\alpha}, \mathfrak{S}^{c}{ }_{\delta}\right\}=\delta_{b}^{c} \mathfrak{L}^{\alpha}{ }_{\delta}+\delta_{\delta}^{\alpha} \mathfrak{R}_{b}^{c}+\delta_{b}^{c} \delta_{\delta}^{\alpha} \mathfrak{C} .
$$

The remaining Lie brackets vanish.

Central extension. This algebra has two further possible central extensions $\mathfrak{P}, \mathfrak{K}$. They are generated by Lie brackets of alike supercharges

$$
\left\{\mathfrak{Q}^{\alpha}{ }_{b}, \mathfrak{Q}^{\gamma}{ }_{d}\right\}=\varepsilon^{\alpha \gamma} \varepsilon_{b d} \mathfrak{P}, \quad\left\{\mathfrak{S}^{a}{ }_{\beta}, \mathfrak{S}^{c}{ }_{\delta}\right\}=\varepsilon^{a c} \varepsilon_{\beta \delta} \mathfrak{K} .
$$

The centrally extended algebra with these charges shall be denoted by

$$
\mathfrak{h}:=\mathfrak{s u}(2 \mid 2) \ltimes \mathbb{R}^{2}=\mathfrak{p s u}(2 \mid 2) \ltimes \mathbb{R}^{3} .
$$

Universal enveloping algebra. The universal enveloping algebra $U(\mathfrak{h})$ of $\mathfrak{h}$ is generated by polynomials of the Lie algebra generators. The Lie brackets are represented as commutators or anti-commutators (depending on the statistics of generators)

$$
[X, Y] \rightarrow X Y-Y X, \quad\{X, Y\} \rightarrow X Y+Y X .
$$

They respect the Lie algebra relations by identification of certain polynomials, e.g.

$$
\mathfrak{Q}_{b}^{\alpha} \mathfrak{Q}_{d}^{\gamma}+\mathfrak{Q}_{d}^{\gamma} \mathfrak{Q}_{b}^{\alpha}=\varepsilon^{\alpha \gamma} \varepsilon_{b d} \mathfrak{P} \text {. }
$$

Chevalley basis. Within the universal enveloping algebra it is not necessary to keep all generators of the Lie algebra explicitly. For example, the central charge $\mathfrak{P}$ can be represented through a quadratic combination of supercharges, see (2.6). A minimal set of generators for this rank-3 algebra is given by three Cartan generators $\mathfrak{H}_{j}$, three simple positive roots $\mathfrak{E}_{j}$ and three simple negative roots $\mathfrak{F}_{j}, j=1,2,3$. One may identify them with the Lie generators as follows:

$$
\begin{aligned}
\mathfrak{H}_{1}=\mathfrak{R}^{2}{ }_{2}-\mathfrak{R}^{1}{ }_{1}=2 \mathfrak{R}^{2}{ }_{2}, & \mathfrak{E}_{1}=\mathfrak{R}^{2}{ }_{1}, & \mathfrak{F}_{1}=\mathfrak{R}^{1}{ }_{2}, \\
\mathfrak{H}_{2}=-\mathfrak{C}-\frac{1}{2} \mathfrak{H}_{1}-\frac{1}{2} \mathfrak{H}_{3}, & \mathfrak{E}_{2}=\mathfrak{Q}^{2}{ }_{2}, & \mathfrak{F}_{2}=\mathfrak{S}^{2}{ }_{2}, \\
\mathfrak{H}_{3}=\mathfrak{L}^{2}{ }_{2}-\mathfrak{L}^{1}{ }_{1}=2 \mathfrak{L}^{2}{ }_{2}, & \mathfrak{E}_{3}=\mathfrak{L}^{1}{ }_{2}, & \mathfrak{F}_{3}=\mathfrak{L}^{2}{ }_{1} .
\end{aligned}
$$

This basis corresponds to the distinguished Dynkin diagram of $\mathfrak{s u}(2 \mid 2)$ in figure 1 . The symmetric Cartan matrix in this basis reads

$$
A_{j k}=\left(\begin{array}{ccc}
+2 & -1 & 0 \\
-1 & 0 & +1 \\
0 & +1 & -2
\end{array}\right)
$$

Note that the algebra $\mathfrak{s u}(2 \mid 2)$ has a degenerate Cartan matrix and therefore has a null vector which reads $v_{j}=(1,2,1)$. It can be used to express the central charge of $\mathfrak{s u}(2 \mid 2)$

$$
\mathfrak{C}=-\frac{1}{2} \sum_{j=1}^{3} v_{j} \mathfrak{H}_{j}=-\frac{1}{2} \mathfrak{H}_{1}-\mathfrak{H}_{2}-\frac{1}{2} \mathfrak{H}_{3} .
$$


The other two central charges in the Chevalley basis take the form

$$
\mathfrak{P}=\left\{\left[\mathfrak{E}_{1}, \mathfrak{E}_{2}\right],\left[\mathfrak{E}_{3}, \mathfrak{E}_{2}\right]\right\}, \quad \mathfrak{K}=\left\{\left[\mathfrak{F}_{1}, \mathfrak{F}_{2}\right],\left[\mathfrak{F}_{3}, \mathfrak{F}_{2}\right]\right\} .
$$

Commutation relations. Let us now state the commutation relations of the universal enveloping algebra. Commutators with the Cartan generators $\mathfrak{H}_{j}$ are given by $(j, k=1,2,3)$

$$
\left[\mathfrak{H}_{j}, \mathfrak{H}_{k}\right]=0, \quad\left[\mathfrak{H}_{j}, \mathfrak{E}_{k}\right]=+A_{j k} \mathfrak{E}_{k}, \quad\left[\mathfrak{H}_{j}, \mathfrak{F}_{k}\right]=-A_{j k} \mathfrak{F}_{k} .
$$

The non-trivial commutators of positive and negative simple roots read

$$
\left[\mathfrak{E}_{1}, \mathfrak{F}_{1}\right]=+\mathfrak{H}_{1}, \quad\left\{\mathfrak{E}_{2}, \mathfrak{F}_{2}\right\}=-\mathfrak{H}_{2}, \quad\left[\mathfrak{E}_{3}, \mathfrak{F}_{3}\right]=-\mathfrak{H}_{3} .
$$

Note that we have normalized the generators in a way such that all relations can be expressed using the symmetric Cartan matrix $A_{j k}$. For superalgebras this leads to a negative sign in $\left[\mathfrak{E}_{j}, \mathfrak{F}_{j}\right]$ for one of the two bosonic subalgebras. The remaining commutators between positive and negative simple roots vanish in this basis

$$
\left[\mathfrak{E}_{j}, \mathfrak{F}_{k}\right]=0 \quad \text { for } \quad j \neq k .
$$

Finally, we need to impose the Serre relations between positive and between negative simple roots.

$$
\begin{aligned}
0 & =\left[\mathfrak{E}_{1}, \mathfrak{E}_{3}\right]=\mathfrak{E}_{2} \mathfrak{E}_{2}=\left[\mathfrak{E}_{1},\left[\mathfrak{E}_{1}, \mathfrak{E}_{2}\right]\right]=\left[\mathfrak{E}_{3},\left[\mathfrak{E}_{3}, \mathfrak{E}_{2}\right]\right] \\
& =\left[\mathfrak{F}_{1}, \mathfrak{F}_{3}\right]=\mathfrak{F}_{2} \mathfrak{F}_{2}=\left[\mathfrak{F}_{1},\left[\mathfrak{F}_{1}, \mathfrak{F}_{2}\right]\right]=\left[\mathfrak{F}_{3},\left[\mathfrak{F}_{3}, \mathfrak{F}_{2}\right]\right] .
\end{aligned}
$$

Note that for superalgebras these standard Serre relations are not sufficient. For the algebra $U(\mathfrak{s u}(2 \mid 2))$ we need two additional Serre relations which effectively read

$$
0=\mathfrak{P}=\mathfrak{K} \text {. }
$$

It is however consistent to drop them altogether which leads to the centrally extended algebra $\mathrm{U}(\mathfrak{h})$.

\subsection{Quantum deformation}

The quantum algebra $\mathrm{U}_{q}(\mathfrak{g})$ is a deformation of the universal enveloping algebra $\mathrm{U}(\mathfrak{g})$ of some Lie algebra $\mathfrak{g}$. It is obtained by inserting various factors and exponents of $q$ in various relations. A convenient combination to use in this context is the so-called quantum number

$$
[x]_{q}:=\frac{q^{x}-q^{-x}}{q-q^{-1}} .
$$

This relation is defined in the same way if $x$ is a generator. There are two ways to achieve this: Either one defines $q^{x}=1+x \log q+\frac{1}{2} x^{2} \log ^{2} q+\cdots$ as a formal power series. For practical purposes one would assume that $q \approx 1$ and thus $\log q \approx 0$. Alternatively one can define $q^{x}$ as an element of the Hopf algebra and $q^{-x}$ as its inverse. This is particularly useful if $x$ appears only with integer multiplicity in exponents, $q^{n x}=\left(q^{x}\right)^{n}$, as will be the case here.

In the previous section we have seen that the two central charges can be obtained by dropping two Serre relations. It is straightforward to apply the same central extension for the quantum deformed algebra $\mathrm{U}_{q}(\mathfrak{s u}(2 \mid 2))$ to obtain $\mathrm{U}_{q}(\mathfrak{h})$.

Commutation Relations. Let us begin with the deformation of the commutation relations of the algebra $\mathrm{U}_{q}(\mathfrak{s u}(2 \mid 2))$. The commutators with Cartan elements do not receive deformations:

$$
\left[\mathfrak{H}_{j}, \mathfrak{H}_{k}\right]=0, \quad\left[\mathfrak{H}_{j}, \mathfrak{E}_{k}\right]=+A_{j k} \mathfrak{E}_{k}, \quad\left[\mathfrak{H}_{j}, \mathfrak{F}_{k}\right]=-A_{j k} \mathfrak{F}_{k} .
$$

The Cartan elements usually appear in exponents, and it is convenient to note the exponentiated form of these relations:

$$
q^{\mathfrak{H}_{j}} \mathfrak{E}_{k}=q^{+A_{j k}} \mathfrak{E}_{k} q^{\mathfrak{H}_{j}}, \quad q^{\mathfrak{H}_{j}} \mathfrak{F}_{k}=q^{-A_{j k}} \mathfrak{F}_{k} q^{\mathfrak{H}_{j}},
$$

6 
The non-trivial commutators of simple roots read in the deformed algebra

$\left[\mathfrak{E}_{1}, \mathfrak{F}_{1}\right]=\left[\mathfrak{H}_{1}\right]_{q}, \quad\left\{\mathfrak{E}_{2}, \mathfrak{F}_{2}\right\}=-\left[\mathfrak{H}_{2}\right]_{q}, \quad\left[\mathfrak{E}_{3}, \mathfrak{F}_{3}\right]=-\left[\mathfrak{H}_{3}\right]_{q}$,

and the remaining mixed commutators vanish

$$
\left[\mathfrak{E}_{j}, \mathfrak{F}_{k}\right]=0 \quad \text { for } \quad j \neq k .
$$

The Serre relations have the same form as in the undeformed algebra (2.14), but with some additional factors of $q$ due to the quantum-adjoint action. Spelled out they yield

$$
\begin{aligned}
0 & =\left[\mathfrak{E}_{1}, \mathfrak{E}_{3}\right]=\left[\mathfrak{F}_{1}, \mathfrak{F}_{3}\right]=\mathfrak{E}_{2} \mathfrak{E}_{2}=\mathfrak{F}_{2} \mathfrak{F}_{2} \\
& =\mathfrak{E}_{1} \mathfrak{E}_{1} \mathfrak{E}_{2}-\left(q+q^{-1}\right) \mathfrak{E}_{1} \mathfrak{E}_{2} \mathfrak{E}_{1}+\mathfrak{E}_{2} \mathfrak{E}_{1} \mathfrak{E}_{1}=\mathfrak{E}_{3} \mathfrak{E}_{3} \mathfrak{E}_{2}-\left(q+q^{-1}\right) \mathfrak{E}_{3} \mathfrak{E}_{2} \mathfrak{E}_{3}+\mathfrak{E}_{2} \mathfrak{E}_{3} \mathfrak{E}_{3} \\
& =\mathfrak{F}_{1} \mathfrak{F}_{1} \mathfrak{F}_{2}-\left(q+q^{-1}\right) \mathfrak{F}_{1} \mathfrak{F}_{2} \mathfrak{F}_{1}+\mathfrak{F}_{2} \mathfrak{F}_{1} \mathfrak{F}_{1}=\mathfrak{F}_{3} \mathfrak{F}_{3} \mathfrak{F}_{2}-\left(q+q^{-1}\right) \mathfrak{F}_{3} \mathfrak{F}_{2} \mathfrak{F}_{3}+\mathfrak{F}_{2} \mathfrak{F}_{3} \mathfrak{F}_{3} .
\end{aligned}
$$

Central Elements. The standard central element from the Cartan subalgebra remains undeformed

$$
\mathfrak{C}=-\frac{1}{2} \mathfrak{H}_{1}-\mathfrak{H}_{2}-\frac{1}{2} \mathfrak{H}_{3} .
$$

As before, the ordinary Serre relations obtained from the Cartan matrix are not sufficient to define $\mathrm{U}_{q}(\mathfrak{s u}(2 \mid 2))$, but we need the constraint $\mathfrak{P}=\mathfrak{K}=0$ with the quantum deformation of (2.10):

$\mathfrak{P}=\mathfrak{E}_{1} \mathfrak{E}_{2} \mathfrak{E}_{3} \mathfrak{E}_{2}+\mathfrak{E}_{2} \mathfrak{E}_{3} \mathfrak{E}_{2} \mathfrak{E}_{1}+\mathfrak{E}_{3} \mathfrak{E}_{2} \mathfrak{E}_{1} \mathfrak{E}_{2}+\mathfrak{E}_{2} \mathfrak{E}_{1} \mathfrak{E}_{2} \mathfrak{E}_{3}-\left(q+q^{-1}\right) \mathfrak{E}_{2} \mathfrak{E}_{1} \mathfrak{E}_{3} \mathfrak{E}_{2}$,

$\mathfrak{K}=\mathfrak{F}_{1} \mathfrak{F}_{2} \mathfrak{F}_{3} \mathfrak{F}_{2}+\mathfrak{F}_{2} \mathfrak{F}_{3} \mathfrak{F}_{2} \mathfrak{F}_{1}+\mathfrak{F}_{3} \mathfrak{F}_{2} \mathfrak{F}_{1} \mathfrak{F}_{2}+\mathfrak{F}_{2} \mathfrak{F}_{1} \mathfrak{F}_{2} \mathfrak{F}_{3}-\left(q+q^{-1}\right) \mathfrak{F}_{2} \mathfrak{F}_{1} \mathfrak{F}_{3} \mathfrak{F}_{2}$.

In the centrally extended algebra $\mathrm{U}_{q}(\mathfrak{h})$ we will not impose the constraint and thus obtain two non-trivial central elements $\mathfrak{P}, \mathfrak{K}$. It is straightforward, if tedious, to confirm that these polynomials are indeed central elements of the quantum-deformed algebra.

\subsection{Representations}

It is commonly the case for a finite-dimensional simple Lie algebra $\mathfrak{g}$ and for generic values of $q$ that the representation theory of the quantum deformed algebra $\mathrm{U}_{q}(\mathfrak{g})$ is analogous to the one of $\mathfrak{g} .{ }^{6}$ We have studied some of the simplest finite-dimensional representations of $\mathrm{U}_{q}(\mathfrak{h})$ and found agreement with this rule. Here we would like to give an overview over some basic representations of $\mathrm{U}_{q}(\mathfrak{h})$ assuming that there is no qualitative difference to the undeformed case.

Outer automorphism. Finite representations of $\mathfrak{h}$ were studied in [17]. The starting point was the representation theory of $\mathfrak{s u}(2 \mid 2)$ with fixed eigenvalue $C_{0}$ of the central charge $\mathfrak{C}$, studied in, e.g. [35, 36]. The SL(2) outer automorphism of $\mathfrak{h}$ then allows us to rotate the triplet of eigenvalues $\left(C_{0}, 0,0\right)$ of the central charges $(\mathfrak{C}, \mathfrak{P}, \mathfrak{K})$ to any desired triplet $(C, P, K)$ with $C_{0}^{2}=C^{2}-P K{ }^{7}$ The representation of the $\mathfrak{p s u}(2 \mid 2)$ generators $\mathfrak{Q}, \mathfrak{S}$ is obtained by conjugation with the $\operatorname{SL}(2)$ element. Note that the combination $\overrightarrow{\mathfrak{C}}^{2}=\mathfrak{C}^{2}-\mathfrak{P} \mathfrak{K}$ is invariant under the automorphism.

It appears that for the quantum-deformed algebra $\mathrm{U}_{q}(\mathfrak{h})$ there also exists a similar outer automorphism. We could use it to relate representations of $\mathrm{U}_{q}(\mathfrak{h})$ to those of $\mathrm{U}_{q}(\mathfrak{s u}(2 \mid 2))$, see [37], which in turn are analogous to those of $\mathfrak{s u}(2 \mid 2)$. We however do not yet understand the automorphism explicitly and therefore the existence of the below representations is an

6 In quantum algebras one singles out the case when $q$ is a root of unity because the representation theory is very special at these points.

7 For simplicity we shall consider the algebra $\mathfrak{h}$ to be complex, and thus there is no distinction between positive and negative values of $C^{2}-P K$. 
educated guess. The above combination $\overrightarrow{\mathfrak{C}}^{2}$ should be quantum-deformed to some $\left[\overrightarrow{\mathfrak{C}}^{2}\right]_{q}$. The classification of representations would then use the eigenvalues of this operator. We find that the smallest representations are indeed characterized by the eigenvalues of the operator

$$
\left[\overrightarrow{\mathfrak{C}}^{2}\right]_{q}:=[\mathfrak{C}]_{q}^{2}-\mathfrak{P} \mathfrak{K} .
$$

Presumably this combination is invariant under the tentative automorphism.

Let us consider typical (long) and atypical (short) representations of $\mathrm{U}_{q}(\mathfrak{h})$ in analogy to the $\mathfrak{s u}(2 \mid 2)$ representations studied in [35].

Long multiplets. The standard finite-dimensional type of representation shall be denoted by

$$
\{m, n ; C, P, K\} \text {. }
$$

It corresponds to the typical highest-weight representations of $\mathfrak{s u}(2 \mid 2)$ with Dynkin labels $[m ; r ; n]$ and $r= \pm\left(\left[\vec{C}^{2}\right]_{q}\right)^{1 / 2}-\frac{1}{2} n+\frac{1}{2} m$. The non-negative integers $n, m$ represent the Dynkin labels of the $\mathfrak{s u}(2) \times \mathfrak{s u}(2)$ subalgebra.

We can decompose a $\mathrm{U}_{q}(\mathfrak{h})$ multiplet into irreducible multiplets of the subalgebra $\mathrm{U}_{q}(\mathfrak{s u}(2) \times \mathfrak{s u}(2))$. Let the symbol $[k]$ represent the $\mathrm{U}_{q}(\mathfrak{s u}(2))$ representation with spin $k / 2$; $[-1]$ is the empty (zero-dimensional) representation. Then the long multiplet decomposes as follows:

$$
\begin{aligned}
\{m, n\} \rightarrow([m] & \otimes[1] \otimes[1],[n]) \oplus([m],[n] \otimes[1] \otimes[1]) \\
& \oplus([m] \otimes[1],[n] \otimes[1]) \oplus([m] \otimes[1],[n] \otimes[1]) .
\end{aligned}
$$

Note the well-known tensor product of $\mathrm{U}_{q}(\mathfrak{s u}(2))$ representations

$$
[m] \otimes[n]=\bigoplus_{k=0}^{\min (m, n)}[m+n-2 k] .
$$

The dimension of a long multiplet is thus $16(m+1)(n+1)$.

Short multiplets. A short multiplet shall be labeled by

$$
\langle m, n ; C, P, K\rangle \text {. }
$$

It can only exist when the constraint

$$
\left[\vec{C}^{2}\right]_{q}=[C]_{q}^{2}-P K=\left[\frac{1}{2}(m+n+1)\right]_{q}^{2}
$$

holds. The decomposition into irreducible representations of $\mathrm{U}_{q}(\mathfrak{s u}(2) \times \mathfrak{s u}(2))$ takes the form

$$
\begin{aligned}
\langle m, n\rangle \rightarrow([m] & \otimes[1],[n]) \oplus([m-1],[n-1] \otimes[1]) \\
\oplus([m],[n] & \otimes[1]) \oplus([m-1] \otimes[1],[n-1]) .
\end{aligned}
$$

This multiplet has dimension $4(m+1)(n+1)+4 m n$. For example, the four-dimensional fundamental representation is the special case $m=n=0$ which decomposes into $\langle 0,0\rangle \rightarrow([1],[0]) \oplus([0],[1])$.

Multiplet splitting. The vector space of a long multiplet $\{m, n ; C, P, K\}$ can be decomposed into the vector spaces of two short multiplets

$$
\{m, n ; \vec{C}\} \rightarrow\langle m+1, n ; \vec{C}\rangle \oplus\langle m, n+1 ; \vec{C}\rangle
$$

However, the typical representation of $\mathrm{U}_{q}(\mathfrak{h})$ is in general irreducible. A special case is when the labels and central charges obey the shortening condition

$$
\left[\vec{C}^{2}\right]_{q}=\left[\frac{1}{2}(m+n+2)\right]_{q}^{2}
$$



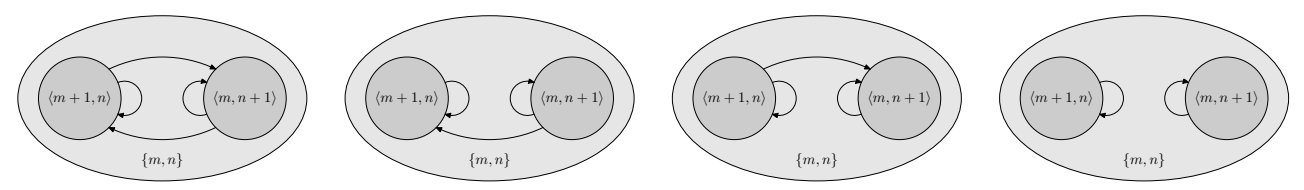

Figure 2. Multiplet splitting. The long multiplet $\{m, n\}$ consists of two short multiplets $\langle m+1, n\rangle$ and $\langle m, n+1\rangle$ and a representation of $\mathrm{U}_{q}(\mathfrak{h})$ maps between the short multiplets (arrows). Generically, the short multiplets are connected in all possible ways (left). When the shortening condition holds one (middle) or both (right) arrows between the short multiplets are broken. In the second figure from the left, $\langle m+1, n\rangle$ is a subrepresentation while $\langle m, n+1\rangle$ is a factor representation, and the long multiplet is indecomposable. The long multiplet in the right figure is fully decomposable.

which is equivalent to the constraint (2.29) for the short multiplets in (2.31). The long representation may then be reduced into the above two short representations. However, in general one cannot expect the long representation to be decomposable, it merely has one short subrepresentation which closes on one of the smaller vector spaces. When projecting out this small vector space one obtains a short factor representation on the other small vector space. This fact is perhaps best illustrated by a figure, see figure 2 .

Tensor products. Let us state some formulae for the decomposition of tensor products of long and short representations. It is convenient to introduce some short-hand notation for sums of similar representations

$$
\{[k] \oplus[l], n\}:=\{k, n\} \oplus\{l, n\}, \quad \text { etc. }
$$

The tensor product of two long representations of $\mathrm{U}_{q}(\mathfrak{h})$ decomposes as follows:

$$
\begin{aligned}
\{m, n\} \otimes\{k, l\}= & \{[m] \otimes[k] \otimes[1] \otimes[1],[n] \otimes[l]\} \\
& \oplus\{[m] \otimes[k] \otimes[1],[n] \otimes[l] \otimes[1]\} \\
& \oplus\{[m] \otimes[k] \otimes[1],[n] \otimes[l] \otimes[1]\} \\
& \oplus\{[m] \otimes[k],[n] \otimes[l] \otimes[1] \otimes[1]\}
\end{aligned}
$$

For a tensor product of a long and a short representation the following decomposition applies

$$
\begin{aligned}
\{m, n\} \otimes\langle k, l\rangle= & \{[m] \otimes[k] \otimes[1],[n] \otimes[l]\} \\
& \oplus\{[m] \otimes[k-1] \otimes[1],[n] \otimes[l-1]\} \\
& \oplus\{[m] \otimes[k],[n] \otimes[l] \otimes[1]\} \\
& \oplus\{[m] \otimes[k-1],[n] \otimes[l-1] \otimes[1]\} .
\end{aligned}
$$

Finally, the tensor product of two short representations reads

$$
\begin{aligned}
\langle m, n\rangle \otimes\langle k, l\rangle= & \{[m] \otimes[k],[n] \otimes[l]\} \\
& \oplus\{[m] \otimes[k-1],[n] \otimes[l-1]\} \\
& \oplus\{[m-1] \otimes[k],[n-1] \otimes[l]\} \\
& \oplus\{[m-1] \otimes[k-1],[n-1] \otimes[l-1]\}
\end{aligned}
$$

These formulae agree with the decompositions (2.26)-(2.30). 


\subsection{Hopf algebra}

Here we will complete the Hopf algebra structure of our quantum deformed algebra. We first define the coproduct and then state the unit, counit and antipode.

Coproduct. We shall use the standard coproduct for the generators of $\mathrm{U}_{q}(\mathfrak{s u}(2 \mid 2))$

$$
\begin{aligned}
& \Delta(1)=1 \otimes 1, \\
& \Delta\left(\mathfrak{H}_{j}\right)=\mathfrak{H}_{j} \otimes 1+1 \otimes \mathfrak{H}_{j}, \\
& \Delta\left(\mathfrak{E}_{j}\right)=\mathfrak{E}_{j} \otimes 1+q^{-\mathfrak{H}_{j}} \otimes \mathfrak{E}_{j}, \\
& \Delta\left(\mathfrak{F}_{j}\right)=\mathfrak{F}_{j} \otimes q^{\mathfrak{H}_{j}}+1 \otimes \mathfrak{F}_{j},
\end{aligned}
$$

where 1 denotes the unit element of the Hopf algebra. Note that the relations are fully compatible with the Serre relations and commutators. The coproducts for the central charges follow by substituting their expressions in terms of the above generators

$$
\begin{aligned}
& \Delta(\mathfrak{C})=\mathfrak{C} \otimes 1+1 \otimes \mathfrak{C}, \\
& \Delta(\mathfrak{P})=\mathfrak{P} \otimes 1+q^{2 \mathfrak{C}} \otimes \mathfrak{P}, \\
& \Delta(\mathfrak{K})=\mathfrak{K} \otimes q^{-2 \mathfrak{C}}+1 \otimes \mathfrak{K} .
\end{aligned}
$$

Note that the coproduct for the generators $\mathfrak{P}, \mathfrak{K}$ turns out to be proportional to the generators themselves as expected. This fact is related to the role of $\mathfrak{P}=\mathfrak{K}=0$ as Serre relations of $\mathrm{U}_{q}(\mathfrak{s u}(2 \mid 2))$. The coproduct of such consistent identifications has to be proportional to these or other identifications.

Unit, counit and antipode. The unit element of the Hopf algebra is denoted by $\eta(1)=1$. The counit $\varepsilon: \mathrm{U}_{q}(\mathfrak{h}) \rightarrow \mathbb{C}$ takes the usual form

$$
\varepsilon(1)=1, \quad \varepsilon\left(\mathfrak{H}_{j}\right)=\varepsilon\left(\mathfrak{E}_{j}\right)=\varepsilon\left(\mathfrak{F}_{j}\right)=0 .
$$

Finally, the antipode $S: \mathrm{U}_{q}(\mathfrak{h}) \rightarrow \mathrm{U}_{q}(\mathfrak{h})$ is uniquely fixed by the compatibility condition

$$
\mu \circ(S \otimes 1) \circ \Delta(\mathfrak{J})=\mu \circ(1 \otimes S) \circ \Delta(\mathfrak{J})=\eta \circ \varepsilon(\mathfrak{J}),
$$

for all $\mathfrak{J} \in \mathrm{U}_{q}(\mathfrak{h})$, where $\eta: \mathbb{C} \rightarrow \mathrm{U}_{q}(\mathfrak{h})$ denotes the unit and $\mu: \mathrm{U}_{q}(\mathfrak{h}) \otimes \mathrm{U}_{q}(\mathfrak{h}) \rightarrow \mathrm{U}_{q}(\mathfrak{h})$ denotes the product. One obtains

$$
S(1)=1, \quad S\left(\mathfrak{H}_{j}\right)=-\mathfrak{H}_{j}, \quad S\left(\mathfrak{E}_{j}\right)=-q^{\mathfrak{H}_{j}} \mathfrak{E}_{j}, \quad S\left(\mathfrak{F}_{j}\right)=-\mathfrak{F}_{j} q^{-\mathfrak{H}_{j}}
$$

and similarly for the central charges

$$
S(\mathfrak{C})=-\mathfrak{C}, \quad S(\mathfrak{P})=-q^{-2 \mathfrak{C}} \mathfrak{P}, \quad S(\mathfrak{K})=-q^{2 \mathfrak{C}} \mathfrak{K} .
$$

Hermitian conjugation. So far we have for simplicity assumed a complex algebra. To restrict to a real algebra we must identify the generators with their conjugates. The proper Hermiticity relations compatible with the coproduct are

$$
\mathfrak{H}_{j}^{\dagger}=\mathfrak{H}_{j}, \quad \mathfrak{E}_{j}^{\dagger}=q^{-\mathfrak{H}_{j}} \mathfrak{F}_{j} .
$$

This implies the following relations for the central charges:

$$
\mathfrak{C}^{\dagger}=\mathfrak{C}, \quad \mathfrak{P}^{\dagger}=q^{2 \mathfrak{C}} \mathfrak{K} .
$$

To have a Hermitian coproduct we furthermore have to constrain $q$ to be real.

Braiding. Just as for the undeformed algebra $\mathrm{U}(\mathfrak{h})$ we shall deform the coproduct slightly and according to a $\mathbb{Z}$-grading of the algebra. This grading associates the charges $+2,+1,-1,-2$ with the generators $\mathfrak{P}, \mathfrak{E}_{2}, \mathfrak{F}_{2}, \mathfrak{K}$, respectively; the other generators are uncharged. This braiding will later lead to a very non-trivial $R$-matrix. 
For the braiding we introduce a new Abelian generator $\mathfrak{U}$ and deform the coproduct (2.37) as follows:

$$
\begin{aligned}
& \Delta\left(\mathfrak{E}_{2}\right)=\mathfrak{E}_{2} \otimes 1+q^{-\mathfrak{H}_{2}} \mathfrak{U} \otimes \mathfrak{E}_{2}, \\
& \Delta\left(\mathfrak{F}_{2}\right)=\mathfrak{F}_{2} \otimes q^{\mathfrak{H}_{2}}+\mathfrak{U}^{-1} \otimes \mathfrak{F}_{2}, \\
& \Delta(\mathfrak{P})=\mathfrak{P} \otimes 1+q^{2 \mathfrak{C}_{\mathfrak{U}}} \otimes \mathfrak{P}, \\
& \Delta(\mathfrak{K})=\mathfrak{K} \otimes q^{-2 \mathfrak{C}}+\mathfrak{U}^{-2} \otimes \mathfrak{K}, \\
& \Delta(\mathfrak{U})=\mathfrak{U} \otimes \mathfrak{U} .
\end{aligned}
$$

The coproduct of the other elements remains undeformed. This deformation of the coproduct is consistent with the algebra because the exponents of $\mathfrak{U}$ follow the $\mathbb{Z}$-grading of the algebra. The counit for $\mathfrak{U}$ should be defined as $\varepsilon(\mathfrak{U})=1$. The antipode must then obey the relation (2.40) which leads to the following modifications of (2.41):

$$
S\left(\mathfrak{E}_{2}\right)=-q^{\mathfrak{H}_{2}} \mathfrak{U}^{-1} \mathfrak{E}_{2}, \quad S\left(\mathfrak{F}_{2}\right)=-q^{-\mathfrak{H}_{2}} \mathfrak{U} \mathfrak{F}_{2}, \quad S(\mathfrak{U})=\mathfrak{U}^{-1}
$$

and for the central charges

$$
S(\mathfrak{C})=-\mathfrak{C}, \quad S(\mathfrak{P})=-q^{-2 \mathfrak{C}} \mathfrak{U}^{-2} \mathfrak{P}, \quad S(\mathfrak{K})=-q^{2 \mathfrak{C}} \mathfrak{U}^{2} \mathfrak{K} .
$$

The Hermitian conjugation remains untouched by the braiding and

$$
\mathfrak{U}^{\dagger}=\mathfrak{U}^{-1} \text {. }
$$

Cocommutativity of the center. A Hopf algebra is called quasi-cocommutative if the coproduct $\Delta(\mathfrak{J})$ and the opposite coproduct $\Delta_{\text {op }}(\mathfrak{J}):=\mathcal{P}(\Delta(\mathfrak{J}))$ are related by conjugation. Here $\mathcal{P}$ defines the graded permutation operator for the tensor product. The conjugation is specified by an $R$-matrix $\mathcal{R} \in \mathrm{U}_{q}(\mathfrak{h}) \otimes \mathrm{U}_{q}(\mathfrak{h})$ satisfying the cocommutativity condition

$$
\Delta_{\text {op }}(\mathfrak{J}) \mathcal{R}=\mathcal{R} \Delta(\mathfrak{J}) \text {. }
$$

For generators $\mathfrak{J}$ from the center of $\mathrm{U}_{q}(\mathfrak{h})$ this relation implies that the coproduct must cocommute $\Delta_{\text {op }}(\mathfrak{J})=\Delta(\mathfrak{J})$. With our coproduct this is obvious for the central charge $\mathfrak{C}$

$$
\Delta(\mathfrak{C})=\mathfrak{C} \otimes 1+1 \otimes \mathfrak{C}=\Delta_{\text {op }}(\mathfrak{C})
$$

The other two central charges $\mathfrak{P}$ and $\mathfrak{K}$ however do not enjoy this property:

$$
\begin{aligned}
& \Delta(\mathfrak{P})=\mathfrak{P} \otimes 1+q^{2 \mathfrak{C}} \mathfrak{U}^{2} \otimes \mathfrak{P}, \quad \text { but } \quad \Delta_{\text {op }}(\mathfrak{P})=\mathfrak{P} \otimes \mathfrak{U}^{2} q^{2 \mathfrak{C}}+1 \otimes \mathfrak{P}, \\
& \Delta(\mathfrak{K})=\mathfrak{K} \otimes q^{-2 \mathfrak{C}}+\mathfrak{U}^{-2} \otimes \mathfrak{K}, \quad \text { but } \quad \Delta_{\text {op }}(\mathfrak{K})=\mathfrak{K} \otimes \mathfrak{U}^{-2}+q^{-2 \mathfrak{C}} \otimes \mathfrak{K} .
\end{aligned}
$$

By taking the difference of the coproducts one can see that the central charges $\mathfrak{P}, \mathfrak{K}$ have to be identified with $\mathfrak{C}$ and $\mathfrak{U}$ as follows:

$$
\mathfrak{P}=g \alpha\left(1-q^{2 \mathfrak{C}} \mathfrak{U}^{2}\right), \quad \mathfrak{K}=g \alpha^{-1}\left(q^{-2 \mathfrak{C}}-\mathfrak{U}^{-2}\right) .
$$

Here $g$ and $\alpha$ are two global constants of the reduced algebra.

An alternative derivation of the braiding and identifications based on a consistent factorized scattering picture are presented in appendix B.

\subsection{Fundamental representation}

The fundamental representation is the short multiplet

$$
\langle C, P, K\rangle=\langle 0,0 ; C, P, K\rangle \text {. }
$$

The vector space is spanned by four states

$$
\left|\phi^{1}\right\rangle,\left|\phi^{2}\right\rangle,\left|\psi^{1}\right\rangle,\left|\psi^{2}\right\rangle,
$$


where $\left|\phi^{a}\right\rangle$ are bosonic and $\left|\psi^{\alpha}\right\rangle$ are fermionic. The fundamental action of the Chevalley-Serre generators is given by

$\begin{array}{llll}\mathfrak{H}_{1}\left|\phi^{1}\right\rangle=-\left|\phi^{1}\right\rangle, & \mathfrak{H}_{2}\left|\phi^{1}\right\rangle=-\left(C-\frac{1}{2}\right)\left|\phi^{1}\right\rangle, & \mathfrak{E}_{1}\left|\phi^{1}\right\rangle=q^{+1 / 2}\left|\phi^{2}\right\rangle, & \mathfrak{F}_{2}\left|\phi^{1}\right\rangle=c\left|\psi^{1}\right\rangle, \\ \mathfrak{H}_{1}\left|\phi^{2}\right\rangle=+\left|\phi^{2}\right\rangle, & \mathfrak{H}_{2}\left|\phi^{2}\right\rangle=-\left(C+\frac{1}{2}\right)\left|\phi^{2}\right\rangle, & \mathfrak{E}_{2}\left|\phi^{2}\right\rangle=a\left|\psi^{2}\right\rangle, & \mathfrak{F}_{1}\left|\phi^{2}\right\rangle=q^{-1 / 2}\left|\phi^{1}\right\rangle, \\ \mathfrak{H}_{3}\left|\psi^{2}\right\rangle=+\left|\psi^{2}\right\rangle, & \mathfrak{H}_{2}\left|\psi^{2}\right\rangle=-\left(C+\frac{1}{2}\right)\left|\psi^{2}\right\rangle, & \mathfrak{E}_{3}\left|\psi^{2}\right\rangle=q^{-1 / 2}\left|\psi^{1}\right\rangle, & \mathfrak{F}_{2}\left|\psi^{2}\right\rangle=d\left|\phi^{2}\right\rangle, \\ \mathfrak{H}_{3}\left|\psi^{1}\right\rangle=-\left|\psi^{1}\right\rangle, & \mathfrak{H}_{2}\left|\psi^{1}\right\rangle=-\left(C-\frac{1}{2}\right)\left|\psi^{1}\right\rangle, & \mathfrak{E}_{2}\left|\psi^{1}\right\rangle=b\left|\phi^{1}\right\rangle, & \mathfrak{F}_{3}\left|\psi^{1}\right\rangle=q^{+1 / 2}\left|\psi^{2}\right\rangle .\end{array}$

The braiding generator $\mathfrak{U}$ will act with the same eigenvalue $U$ on all four states.

Constraints. It is not too hard to see that the closure of the algebra of supercharges leads to the constraints

$$
a d=\left[C+\frac{1}{2}\right]_{q}, \quad b c=\left[C-\frac{1}{2}\right]_{q}, \quad a b=P, \quad c d=K .
$$

This is in agreement with the constraint (2.29) which is required for the fundamental representation

$$
\left[\vec{C}^{2}\right]_{q}=\left[\frac{1}{2}\right]_{q}^{2}
$$

It furthermore follows that the constraint $a d-b c=1$ for $q=1$ is deformed to

$$
(a d-q b c)\left(a d-q^{-1} b c\right)=1 .
$$

Note that there is a subtlety in taking the limit $q \rightarrow 1$ in the above constraint (2.58): it leads to two inequivalent constraints $a d-b c= \pm 1$ which correspond to different embeddings of the Lie algebra into the Chevalley basis. The plus sign yields the above constraint with the identifications in (2.7) or equivalently $\mathfrak{E}_{2}=-\mathfrak{Q}^{2}{ }_{2}, \mathfrak{F}_{2}=-\mathfrak{S}^{2}{ }_{2}$. The minus sign corresponds to one of the following embeddings:

$$
\mathfrak{E}_{2}=\mp \mathfrak{Q}^{2}{ }_{2}, \quad \mathfrak{F}_{2}= \pm \mathfrak{S}^{2}{ }_{2}, \quad \mathfrak{H}_{2}=\mathfrak{C}+\frac{1}{2} \mathfrak{H}_{1}+\frac{1}{2} \mathfrak{H}_{3} .
$$

$x^{ \pm}$Parameters. For later convenience we shall introduce the new representation parameters $x^{ \pm}, \gamma$ and rewrite $a, b, c, d$ as follows:

$$
\begin{aligned}
& a=\sqrt{g} \gamma, \\
& b=\frac{\sqrt{g} \alpha}{\gamma} \frac{1}{x^{-}}\left(x^{-}-q^{2 C-1} x^{+}\right), \\
& c=\frac{\mathrm{i} \sqrt{g} \gamma}{\alpha} \frac{q^{-C+1 / 2}}{x^{+}}, \\
& d=\frac{\mathrm{i} \sqrt{g}}{\gamma} q^{C+1 / 2}\left(x^{-}-q^{-2 C-1} x^{+}\right) .
\end{aligned}
$$

In terms of these parameters the constraint (2.56) implies the following quadratic relation between $x^{ \pm}$:

$$
\frac{x^{+}}{q}+\frac{q}{x^{+}}-q x^{-}-\frac{1}{q x^{-}}+\mathrm{i} g\left(q-q^{-1}\right)\left(\frac{x^{+}}{q x^{-}}-\frac{q x^{-}}{x^{+}}\right)=\frac{\mathrm{i}}{g} .
$$

The central charge $C$ cannot be written unambiguously using $x^{ \pm}$, but the combination $q^{2 C}$ is well defined:

$$
q^{2 C}=q \frac{\left(q-q^{-1}\right) / x^{+}-\mathrm{i} g^{-1}}{\left(q-q^{-1}\right) / x^{-}-\mathrm{i} g^{-1}}=q^{-1} \frac{\left(q-q^{-1}\right) x^{+}+\mathrm{i} g^{-1}}{\left(q-q^{-1}\right) x^{-}+\mathrm{i} g^{-1}} .
$$


These two expressions are equivalent upon (2.61). Finally, the central charges $P, K$ read

$$
P=g \alpha\left(1-q^{2 C} \frac{x^{+}}{q x^{-}}\right), \quad K=g \alpha^{-1}\left(q^{-2 C}-\frac{q x^{-}}{x^{+}}\right) .
$$

With the above identification (2.52) of $\mathfrak{P}$ and $\mathfrak{K}$ with $\mathfrak{U}$, the squared eigenvalue of the latter on the fundamental representation reads

$$
U^{2}=\frac{x^{+}}{q x^{-}}
$$

Fermion normalization. The parameter $\gamma$ adjusts the normalization of fermions $\left|\psi^{\alpha}\right\rangle$ with respect to bosons $\left|\phi^{a}\right\rangle$. Furthermore the parameter $\alpha$ adjusts the normalization of $\mathfrak{E}_{2}, \mathfrak{P}$ versus $\mathfrak{F}_{2}, \mathfrak{K}$. A particularly useful choice for $\gamma$ will turn out to be

$$
\gamma=\frac{\sqrt{-\mathrm{i} \alpha q^{C+1 / 2} U\left(x^{+}-x^{-}\right)}}{\sqrt[4]{1-\left(q-q^{-1}\right)^{2} g^{2}}} .
$$

Apart from the features discussed below, we expect it to have nice analytic properties analogous to the case of the undeformed case discussed in [38]. Despite some simplifications we will largely keep $\alpha$ and $\gamma$ unspecified in this paper.

Uniformization. For a fixed parameter $g$ the constraint (2.61) defines a complex torus with modulus

$$
k=4 \mathrm{i} g \sqrt{1-g^{2}\left(q-q^{-1}\right)^{2}} .
$$

The quotient of the parameters $x^{ \pm}$can be expressed conveniently through the point $z$ on a doubly-periodic complex plane using

$$
\frac{x^{+}}{q x^{-}}=\frac{\mathrm{cn}(z)+\mathrm{i} r \operatorname{sn}(z)}{\operatorname{cn}(z)-\mathrm{i} r \operatorname{sn}(z)},
$$

where the constant $r$ is given by

$$
r=\sqrt{\frac{1+16 g^{2}-16 g^{4}\left(q-q^{-1}\right)^{2}}{1+4 g^{2}\left(q^{1 / 2}+q^{-1 / 2}\right)^{2}}} .
$$

The individual parameters $x^{ \pm}$are then written as rational functions of $\operatorname{sn}(z), \operatorname{cn}(z), \operatorname{dn}(z)$, but we shall refrain from stating these functions explicitly here.

Transposition. The antipode map corresponds to transposing the representation matrices

$$
S(\mathfrak{J}) \simeq \mathcal{C}^{-1} \overline{\mathfrak{J}}^{\text {sT }} \mathcal{C},
$$

where the supertransposition is defined by $A_{j k}^{\mathrm{sT}}:=(-1)^{(|j|+1)|k|} A_{k j}$ and $\mathcal{C}$ is the charge conjugation matrix

$$
\begin{array}{ll}
\mathcal{C}\left|\phi^{1}\right\rangle=-q^{+1 / 2}\left|\phi^{2}\right\rangle, & \mathcal{C}\left|\psi^{1}\right\rangle=-q^{+1 / 2}\left|\psi^{2}\right\rangle, \\
\mathcal{C}\left|\phi^{2}\right\rangle=+q^{-1 / 2}\left|\phi^{1}\right\rangle, & \mathcal{C}\left|\psi^{2}\right\rangle=+q^{-1 / 2}\left|\psi^{1}\right\rangle .
\end{array}
$$

The conjugation matrix was constructed such that the equation is satisfied for the bosonic Chevalley-Serre generators $\mathfrak{E}_{1}, \mathfrak{E}_{3}, \mathfrak{F}_{1}, \mathfrak{F}_{3}$. By solving this equation for the fermionic generators $\mathfrak{E}_{2}, \mathfrak{F}_{2}$, we obtain the antipode representation parameters $\bar{a}, \bar{b}, \bar{c}, \bar{d}$ in terms of the original parameters $a, b, c, d$ (see also [15]):

$$
\begin{aligned}
& \bar{a}=-U^{-1} q^{-C+1 / 2} b, \\
& \bar{b}=U^{-1} q^{-C-1 / 2} a, \\
& \bar{c}=-U q^{C+1 / 2} d, \\
& \bar{d}=U q^{C-1 / 2} c .
\end{aligned}
$$


We can solve these equations for the crossed spectral parameters and obtain ${ }^{8}$

$$
\bar{x}^{+}=s\left(x^{+}\right), \quad \bar{x}^{-}=s\left(x^{-}\right), \quad \bar{\gamma}=\frac{q^{1 / 2} \alpha}{\gamma}\left(q^{C} U-q^{-C} U^{-1}\right) .
$$

Note that $\bar{\gamma}$ is compatible with the choice (2.65) of $\gamma$ as a function of $x^{ \pm}$. The antipode map $s(x)$ for the parameters $x^{ \pm}$is defined as

$$
s(x)=\frac{\mathrm{i} g^{-1}+\left(q-q^{-1}\right) x}{\mathrm{i} g^{-1} x-\left(q-q^{-1}\right)} .
$$

This map $x^{ \pm} \mapsto s\left(x^{ \pm}\right)$acts on the braiding factor and the central charge as follows:

$$
\bar{C}=-C, \quad \bar{U}=\frac{1}{U} .
$$

Hermiticity. Let us introduce the canonical scalar product for our states

$$
\left\langle\phi_{a} \mid \phi^{b}\right\rangle=\delta_{a}^{b}, \quad\left\langle\psi_{\alpha} \mid \psi^{\beta}\right\rangle=\delta_{\alpha}^{\beta} .
$$

The representation is Hermitian if the conditions in (2.43) hold. They imply the relations $a^{*}=q^{C+1 / 2} d$ and $b^{*}=q^{C-1 / 2} c$. Expressed in terms of $x^{ \pm}$-parameters they correspond to

$$
\left(x^{+}\right)^{*}=\frac{1}{s\left(x^{-}\right)}, \quad\left(x^{-}\right)^{*}=\frac{1}{s\left(x^{+}\right)} .
$$

This is equivalent to the condition that the uniformized parameter $z$ on the complex torus is real. Furthermore the moduli of the parameters $\gamma$ and $\alpha$ are constrained,

$$
|\gamma|^{2}=-\mathrm{i} x^{+}+\mathrm{i} q^{2 C+1} x^{-}, \quad|\alpha|^{2}=1 .
$$

Again, the first constraint is automatically satisfied when $\gamma$ is given by (2.65) as a function of $x^{ \pm}$

\section{The fundamental $R$-matrix}

To determine the full universal $R$-matrix is a formidable task. Here we will satisfy ourselves with the lesser task of finding the representation of the $R$-matrix on two fundamentals.

\subsection{Matrix structure}

We construct the fundamental $R$-matrix by demanding that it satisfies the cocommutativity relation (2.49)

$$
\Delta_{\text {op }}(\mathfrak{J}) \mathcal{R}=\mathcal{R} \Delta(\mathfrak{J})
$$

The most general ansatz which satisfies the relation for the $\mathrm{U}_{q}(\mathfrak{s u}(2) \times \mathfrak{s u}(2))$ subalgebra, i.e. for $\mathfrak{E}_{1}, \mathfrak{E}_{3}, \mathfrak{F}_{1}, \mathfrak{F}_{3}$, is given in table 1 . The ten functions $A, B, C, D, E, F, G, H, K, L$ are $a$ priori free. They are however fully constrained up to one overall phase factor $R_{12}^{0}$ by cocommutativity with respect to the supersymmetry generators $\mathfrak{E}_{2}, \mathfrak{F}_{2}$. This finding is related to the irreducibility of the tensor product of two fundamental representations (2.36):

$$
\langle 0,0\rangle \otimes\langle 0,0\rangle=\{0,0\} .
$$

We present our findings for the ten functions in table 2. This derivation parallels completely the case of the undeformed algebra in [17] and for $q=1$ the results agree. Note that $C_{12}=F_{12}$ and $H_{12}=K_{12}$ if $\gamma$ is determined by (2.65).

${ }^{8}$ Note that $\overline{\bar{\gamma}}=-\gamma$ which is consistent with the $\mathbb{Z}_{4}$-periodicity of supertransposition. Alternatively one could define $\bar{U}=-1 / U$ leading to $\overline{\bar{\gamma}}=\gamma$. 
Table 1. The fundamental $R$-matrix of $\mathrm{U}_{q}(\mathfrak{h})$.

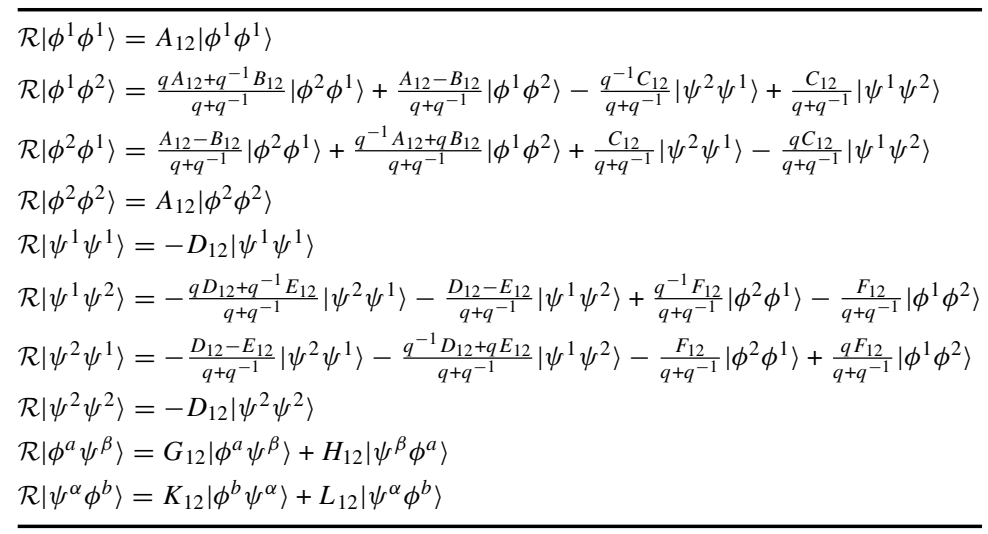

Table 2. The coefficients for the fundamental $R$-matrix of $\mathrm{U}_{q}(\mathfrak{h})$.

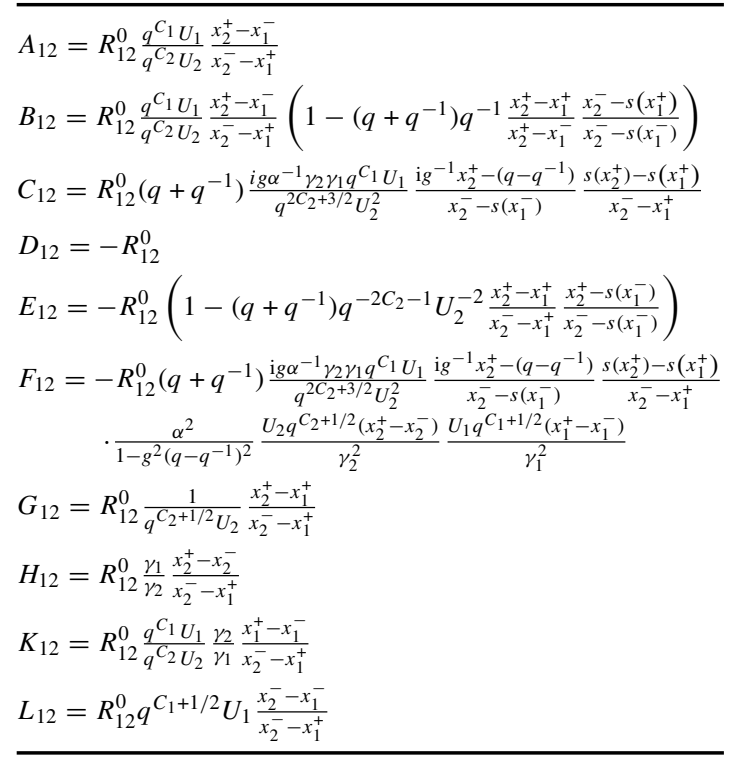

\subsection{Discrete symmetries of the R-matrix}

Braiding unitarity. An $R$-matrix is expected to obey the so-called unitarity condition

$$
\mathcal{R}_{12} \mathcal{R}_{21}=1 \otimes 1
$$

This condition says that the operation of flipping the order of two sites along a chain is an involution. Unitarity implies the following ten relations for the coefficients of the operator in table 1

$$
A_{12} A_{21}=B_{12} B_{21}+C_{12} F_{21}=G_{12} L_{21}+H_{12} H_{21}=1
$$

as well as

$$
\begin{aligned}
& \frac{A_{12}}{D_{21}}=\frac{D_{12}}{A_{21}}, \quad \frac{B_{12}}{E_{21}}=\frac{E_{12}}{B_{21}}=-\frac{C_{12}}{C_{21}}=-\frac{F_{12}}{F_{21}}, \\
& \frac{H_{12}}{K_{21}}=\frac{K_{12}}{H_{21}}=-\frac{G_{12}}{G_{21}}=-\frac{L_{12}}{L_{21}} .
\end{aligned}
$$


Given the $R$-matrix coefficients in table 2 it is easy to convince ourselves that this property indeed holds if the undetermined factor $R_{12}^{0}$ obeys the equation $R_{12}^{0} R_{21}^{0}=1$. Curiously, the coefficients in table 2 even satisfy the stronger relation that all three terms in (3.5) are actually equal. This amounts to

$$
A_{12} D_{12}=B_{12} E_{12}-C_{12} F_{12}=H_{12} K_{12}-G_{12} L_{12} .
$$

Yang-Baxter equation. Furthermore we have considered the Yang-Baxter equation

$$
\mathcal{R}_{12} \mathcal{R}_{13} \mathcal{R}_{23}=\mathcal{R}_{23} \mathcal{R}_{13} \mathcal{R}_{12}
$$

It amounts to around hundred cubic equations among the coefficients $A, \ldots, L$. We have confirmed in Mathematica that all relations hold subject to the constraint (2.61). An alternative argument [17] for the validity of (3.7) uses the decomposition of the threefold tensor product of fundamental multiplets, see (2.35) and (2.36),

$$
\langle 0,0\rangle \otimes\langle 0,0\rangle \otimes\langle 0,0\rangle=\{1,0\} \oplus\{0,1\} .
$$

Effectively we have to prove the YBE only for one component in each of the resulting multiplets. Representative states for the two multiplets are given by $\left|\phi^{1} \phi^{1} \phi^{1}\right\rangle$ and $\left|\psi^{1} \psi^{1} \psi^{1}\right\rangle$. The YBE is trivially satisfied for both and thus it is valid in general.

Matrix unitarity. The $R$-matrix is also a unitary matrix

$$
\left(\mathcal{R}_{12}\right)^{\dagger} \mathcal{R}_{12}=1 \otimes 1
$$

Using the above unitarity we can rewrite the condition as $\left(\mathcal{R}_{12}\right)^{\dagger}=\mathcal{R}_{21}$ which makes it straightforward to read off unitarity conditions for the coefficients

$$
\begin{aligned}
& \left(A_{12}\right)^{*}=A_{21}, \quad\left(B_{12}\right)^{*}=B_{21}, \quad\left(C_{12}\right)^{*}=F_{21}, \quad\left(G_{12}\right)^{*}=L_{21}, \quad\left(H_{12}\right)^{*}=H_{21}, \\
& \left(D_{12}\right)^{*}=D_{21}, \quad\left(E_{12}\right)^{*}=E_{21}, \quad\left(F_{12}\right)^{*}=C_{21}, \quad\left(L_{12}\right)^{*}=G_{21}, \quad\left(K_{12}\right)^{*}=K_{21} \text {. }
\end{aligned}
$$

These conditions are satisfied when the conjugate parameters are given by (2.76) and when the phase factor is a pure phase $\left(R_{12}^{0}\right)^{*}=R_{21}^{0}=\left(R_{12}^{0}\right)^{-1}$.

Crossing symmetry. Finally, the $R$-matrix may in principle have crossing symmetry. The crossing equation for the fundamental $R$-matrix takes the standard form

$$
\left(\mathcal{C}^{-1} \otimes 1\right) \mathcal{R}_{\overline{1} 2}^{\mathrm{sT} \otimes 1}(\mathcal{C} \otimes 1) \mathcal{R}_{12}=1 \otimes 1
$$

Using the same trick as above for matrix unitarity, we can write the crossing relation in terms of the $R$-matrix coefficients as follows:

$$
\begin{aligned}
& A_{\overline{12}}=\frac{A_{21}-B_{21}}{q+q^{-1}}, \quad \frac{A_{\overline{12}}-B_{\overline{12}}}{q+q^{-1}}=A_{21}, \quad G_{\overline{12}}=L_{21}, \quad \frac{C_{\overline{12}}}{q+q^{-1}}=H_{21}, \\
& H_{\overline{12}}=\frac{F_{21}}{q+q^{-1}}, \quad D_{\overline{12}}=\frac{D_{21}-E_{21}}{q+q^{-1}}, \quad \frac{D_{\overline{12}}-E_{\overline{12}}}{q+q^{-1}}=D_{21}, \\
& L_{\overline{12}}=G_{21}, \quad \frac{F_{\overline{12}}}{q+q^{-1}}=K_{21}, \quad K_{\overline{12}}=\frac{C_{21}}{q+q^{-1}} .
\end{aligned}
$$

All these relations hold simultaneously if the phase factor satisfies the crossing relation

$$
\begin{aligned}
R_{12}^{0} R_{\overline{12}}^{0} & =q \frac{x_{1}^{-}-x_{2}^{-}}{x_{1}^{-}-x_{2}^{+}} \frac{x_{1}^{+}-s\left(x_{2}^{-}\right)}{x_{1}^{+}-s\left(x_{2}^{+}\right)}=q^{-1} \frac{x_{1}^{+}-x_{2}^{+}}{x_{1}^{-}-x_{2}^{+}} \frac{s\left(x_{1}^{+}\right)-x_{2}^{-}}{s\left(x_{1}^{-}\right)-x_{2}^{-}} \\
& =q \frac{s\left(x_{1}^{-}\right)-s\left(x_{2}^{-}\right)}{s\left(x_{1}^{-}\right)-s\left(x_{2}^{+}\right)} \frac{s\left(x_{1}^{+}\right)-x_{2}^{-}}{s\left(x_{1}^{+}\right)-x_{2}^{+}}=q^{-1} \frac{s\left(x_{1}^{+}\right)-s\left(x_{2}^{+}\right)}{s\left(x_{1}^{-}\right)-s\left(x_{2}^{+}\right)} \frac{x_{1}^{+}-s\left(x_{2}^{-}\right)}{x_{1}^{-}-s\left(x_{2}^{-}\right)},
\end{aligned}
$$

which can be written in many (more) alternative ways. This is the quantum-deformed analog of the crossing relation obtained in [39]. It would be interesting to find a solution of (3.13). 


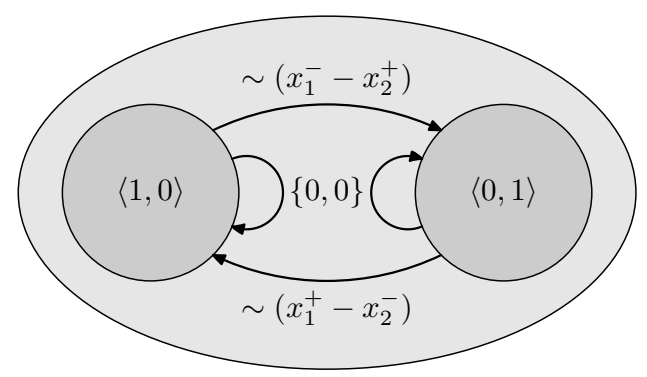

Figure 3. Tensor product structure of two fundamental representations along a chain. $\langle 1,0\rangle$ contains the state $\left|\phi^{1} \phi^{1}\right\rangle$ while $\langle 0,1\rangle$ contains the state $\left|\psi^{1} \psi^{1}\right\rangle$. The arrows indicate the action of the long representation. The long arrows break when $x_{2}^{+}=x_{1}^{-}$or $x_{2}^{-}=x_{1}^{+}$, respectively.

\subsection{Special points}

Let us investigate the behavior of the $R$-matrix at special points of the parameters $x_{1,2}^{ \pm}$. The analysis is analogous to the undeformed case in [17, 40], here we merely give a brief summary.

Permutation. As usual when the representation parameters coincide, $x_{1}^{ \pm}=x_{2}^{ \pm}$, the $R$-matrix becomes a graded permutation operator times -1 .

Standard poles and zeros. The $R$-matrix has a pole at $x_{2}^{+}=x_{1}^{-}$and a zero at $x_{2}^{-}=x_{1}^{+}$. At these points the shortening condition (2.32) holds, and thus the long multiplet in (3.2) splits up as follows (2.31):

$$
\{0,0\} \rightarrow\langle 1,0\rangle \oplus\langle 0,1\rangle
$$

Note that the action of $\mathrm{U}_{q}(\mathfrak{h})$ on two fundamental multiplets is defined via the coproduct, and typically the resulting long representation is not decomposable, see figure 3. In particular, at $x_{2}^{+}=x_{1}^{-}$the representation closes on the short multiplet $\langle 1,0\rangle$ which contains the state $\left|\phi^{1} \phi^{1}\right\rangle$. The residue of the corresponding pole of the $R$-matrix projects to this submultiplet. Conversely at $x_{2}^{-}=x_{1}^{+}$the representation closes on the short multiplet $\langle 0,1\rangle$ which contains the state $\left|\psi^{1} \psi^{1}\right\rangle$. The $R$-matrix projects to this submultiplet at this point. This behavior is in fact standard for Yangian and quantum affine algebras, but here it appears already at the level of the finite-dimensional algebra $\mathrm{U}_{q}(\mathfrak{h})$.

Singlet pole. Furthermore, there is a pole at $x_{2}^{ \pm}=s\left(x_{1}^{ \pm}\right)$whose residues project to the singlet state $|\mathbf{1}\rangle$ discussed below. It corresponds to the decomposition

$$
\{0,0\} \rightarrow \bullet \oplus \mathbf{a d j}_{\mathfrak{p s u}(2 \mid 2)} \oplus \bullet,
$$

where $\bullet$ represents a singlet.

\subsection{Singlet state and quasi-triangularity}

A singlet state is annihilated by all generators of the Hopf algebra $\mathrm{U}_{q}(\mathfrak{h})$. Consequently, a universal $R$-matrix in $\mathrm{U}_{q}(\mathfrak{h}) \otimes \mathrm{U}_{q}(\mathfrak{h})$ would have to act trivially on this state. In a quasitriangular Hopf algebra this also applies to singlet states which are composed from non-singlets 
(fusion). We can use this property to obtain an alternative derivation of the crossing relation (3.13) for the phase factor $R_{12}^{0}$ as in [13].

Singlet state. The singlet component $|\mathbf{1}\rangle$ in a two-spin state is defined by $\Delta(\mathfrak{J})|\mathbf{1}\rangle=0$ which should hold for all generators $\mathfrak{J}$. Without specifying the spin orientations in $|\mathbf{1}\rangle$, the constraints $\Delta(\mathfrak{J}) \simeq 0$ for the central charges $\mathfrak{C}, \mathfrak{P}, \mathfrak{K}$ lead to a relation between the representation labels

$$
x_{1}^{+}=s\left(x_{2}^{+}\right), \quad x_{1}^{-}=s\left(x_{2}^{-}\right) .
$$

We make an ansatz which satisfies the constraint for the bosonic generators $\mathfrak{E}_{1}, \mathfrak{E}_{3}, \mathfrak{F}_{1}, \mathfrak{F}_{3}$

$$
|\mathbf{1}\rangle=q^{-1 / 2}\left|\phi^{1} \phi^{2}\right\rangle-q^{+1 / 2}\left|\phi^{2} \phi^{1}\right\rangle+\kappa q^{-1 / 2}\left|\psi^{1} \psi^{2}\right\rangle-\kappa q^{+1 / 2}\left|\psi^{2} \psi^{1}\right\rangle .
$$

The coefficient $\kappa$ is determined by the constraint for the fermionic generators $\mathfrak{E}_{2}, \mathfrak{F}_{2}$

$$
\kappa=\frac{\gamma_{1} \gamma_{2} q^{-1 / 2}}{\alpha\left(q^{C_{1}} U_{1}-q^{C_{2}} U_{2}\right)} .
$$

By means of the crossing relation (2.72) for $\gamma_{2}=\bar{\gamma}_{1}$ it simply equals $\kappa=1$.

Representation of the R-Matrix. The singlet state obeys the curious identity

$$
\mathcal{R}_{13} \mathcal{R}_{23}\left|\mathbf{1}_{12} X_{3}\right\rangle=\lambda\left|\mathbf{1}_{12} X_{3}\right\rangle
$$

with a common eigenvalue $\lambda$ for all $X \in\left\{\phi^{a}, \psi^{\alpha}\right\}$. The eigenvalue takes the form

$$
\begin{aligned}
\lambda & =\frac{A_{13}\left(A_{23}-B_{23}\right)}{q+q^{-1}}=\frac{D_{13}\left(D_{23}-E_{23}\right)}{q+q^{-1}} \\
& =R_{13}^{0} R_{23}^{0} q^{-1} \frac{q^{C_{1}} U_{1}}{q^{C_{3}} U_{3}} \frac{q^{C_{2}} U_{2}}{q^{C_{3}} U_{3}} \frac{x_{3}^{+}-x_{1}^{-}}{x_{3}^{-}-x_{1}^{-}} \frac{x_{3}^{+}-x_{2}^{+}}{x_{3}^{-}-x_{2}^{+}} .
\end{aligned}
$$

This observation is in agreement with quasi-triangularity of the Hopf algebra $\mathrm{U}_{q}(\mathfrak{h})$ : If we view the two-particle state as transforming under one representation (the singlet) then the product $\mathcal{R}_{13} \mathcal{R}_{23}$ must equal the representation $\mathcal{R}_{13}$ of the $R$-matrix on a singlet and a fundamental (fusion). However, singlet representations of the $R$-matrix are trivial and thus the eigenvalue must be $\lambda=1$. This condition leads to a constraint on the overall phase factor

$$
R_{12}^{0} R_{12}^{0}=q \frac{x_{1}^{-}-x_{2}^{-}}{x_{1}^{-}-x_{2}^{+}} \frac{x_{1}^{+}-s\left(x_{2}^{-}\right)}{x_{1}^{+}-s\left(x_{2}^{+}\right)} .
$$

It is the same as the crossing relation obtained earlier in (3.13). Note that we have mapped the representations according to $(1,2,3) \rightarrow(1, \overline{1}, 2)$. The map $(1,2,3) \rightarrow(\overline{1}, 1,2)$ leads to a contradictory result. A consistent result is achieved by $(1,2,3) \rightarrow(\overline{1}, \overline{\overline{1}}, 2)$ for which we obtain

$$
R_{\overline{12}}^{0} R_{\overline{\overline{1} 2}}^{0}=q \frac{s\left(x_{1}^{-}\right)-x_{2}^{-}}{s\left(x_{1}^{-}\right)-x_{2}^{+}} \frac{s\left(x_{1}^{+}\right)-s\left(x_{2}^{-}\right)}{s\left(x_{1}^{+}\right)-s\left(x_{2}^{+}\right)} .
$$

Note that $R_{\overline{\overline{1}} 2}^{0} \neq R_{12}^{0}$, more precisely there is a double crossing relation which is the quantumdeformed analog of the relation solved in [41]:

$$
\frac{R_{\overline{1} 2}^{0}}{R_{12}^{0}}=\frac{x_{1}^{-}-x_{2}^{+}}{x_{1}^{-}-x_{2}^{-}} \frac{s\left(x_{1}^{-}\right)-x_{2}^{-}}{s\left(x_{1}^{-}\right)-x_{2}^{+}} \frac{x_{1}^{+}-s\left(x_{2}^{+}\right)}{x_{1}^{+}-s\left(x_{2}^{-}\right)} \frac{s\left(x_{1}^{+}\right)-s\left(x_{2}^{-}\right)}{s\left(x_{1}^{+}\right)-s\left(x_{2}^{+}\right)} .
$$




\section{Diagonalizing the $\boldsymbol{R}$-matrix}

The $R$-matrix can be used to determine the eigenstates for certain spin chains. In particular, it is required to write down quantization conditions for eigenstates of finite closed or open spin chains. However, as the $R$-matrix is a matrix, the resulting equations would be matrix equations and rather hard to handle. Instead of dealing with matrix equations we can first 'diagonalize' the $R$-matrix by introducing a suitable vacuum state and excitations. This is done by means of the nested coordinate Bethe ansatz [42], and the procedure follows along the lines of [17] for the undeformed setup. Alternatively, one may perform a nested algebraic Bethe ansatz similar to the one in $[5,43]$ which should lead to the same set of Bethe equations.

\subsection{Vacuum and propagation}

We would like to find suitable eigenstates of a chain of $K$ fundamental representations with labels $x_{k}^{ \pm}, k=1, \ldots, K$. We define the level-II vacuum state $|0\rangle^{\mathrm{II}}$ to consist only of $\phi^{1}$ 's. The top-level $R$-matrix $\mathcal{R}_{\pi}^{\mathrm{I}}$ is a product of pairwise $R$-matrices $\mathcal{R}_{k, j}^{\mathrm{I}}=\mathcal{R}_{k, j}$ representing a permutation $\pi \in S_{K}$ of the $K$ sites. It multiplies the vacuum state by a phase factor

$$
\mathcal{R}_{\pi}^{\mathrm{I}}|0\rangle^{\mathrm{II}}=R_{\pi}^{\mathrm{I}}|0\rangle^{\mathrm{II}} \text {. }
$$

The total phase factor is a product over pairwise phase factors

$R_{\pi}^{\mathrm{I}}=\prod_{(k, j) \in \pi} R^{\mathrm{I}, \mathrm{I}}\left(x_{k}, x_{j}\right), \quad R^{\mathrm{I}, \mathrm{I}}\left(x_{k}, x_{j}\right)=A\left(x_{k}, x_{j}\right)=R^{0}\left(x_{k}, x_{j}\right) \frac{q^{C_{k}} U_{k}}{q^{C_{j}} U_{j}} \frac{x_{j}^{+}-x_{k}^{-}}{x_{j}^{-}-x_{k}^{+}}$.

The level-II vacuum has two flavors of elementary excitations. An excitation replaces one of the $\phi^{1}$ spins by a $\psi^{\alpha}$ spin

$$
\left|\psi^{\alpha}\right\rangle_{\pi}^{\mathrm{II}}=\sum_{k=1}^{K} \Psi_{\pi, k}(y) \mid \phi^{1} \ldots \begin{gathered}
\begin{array}{c}
\pi(k) \\
\downarrow \\
\psi^{\alpha}
\end{array} \\
\left.\ldots \phi^{1}\right\rangle^{\mathrm{I}} .
\end{gathered}
$$

The wavefunction $\Psi_{\pi, k}(y)$ is parametrized by a rapidity $y$ and it depends locally on the parameters $x_{k}^{ \pm}$of the spin representations

$$
\Psi_{\pi, k}(y)=f\left(y, x_{\pi(k)}\right) \prod_{j=1}^{k-1} R^{\mathrm{II}, \mathrm{I}}\left(y, x_{\pi(j)}\right) .
$$

The permutation $\pi$ defines the ordering of the top-level spins along the chain.

In the nested Bethe ansatz the wavefunction must be compatible with the action of the $R$-matrix

$$
\mathcal{R}_{\pi}^{\mathrm{I}}\left|\psi^{\alpha}\right\rangle^{\mathrm{II}}=R_{\pi}^{\mathrm{I}}\left|\psi^{\alpha}\right\rangle_{\pi}^{\mathrm{II}}
$$

It suffices to consider a chain with two sites and the permutation $\pi$ interchanges them. The solution to the compatibility condition reads

$$
R^{\mathrm{II}, \mathrm{I}}\left(y, x_{k}\right)=q^{C_{k}+1 / 2} U_{k} \frac{y-x_{k}^{-}}{y-x_{k}^{+}}, \quad f\left(y, x_{k}\right)=\frac{y \gamma_{k}}{y-x_{k}^{+}} .
$$

Clearly the rapidity $y$ could be replaced by any function of $y$; we chose it such that the functions $R^{\mathrm{II}, \mathrm{I}}\left(y, x_{k}\right)$ and $f\left(y, x_{k}\right)$ are rational. Furthermore $f\left(y, x_{k}\right)$ could be multiplied by any function of $y$. 


\subsection{Scattering}

We now have to solve the analog of the compatibility condition (4.5) for a two-excitation state. We make the following ansatz

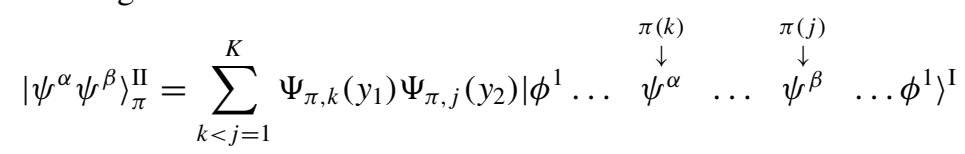

which solves the compatibility condition except when the two excitations are neighbors. This state can mix with a state where one spin $\phi^{1}$ is replaced by $\phi^{2}$

$$
\left|\phi^{2}\right\rangle_{\pi}^{\mathrm{II}}=\sum_{k=1}^{K} \Psi_{\pi, k}\left(y_{1}\right) \Psi_{\pi, k}\left(y_{2}\right) f\left(y_{1}, y_{2}, x_{\pi(k)}\right)\left|\phi^{1} \ldots \phi^{2} \quad \ldots \phi^{1}\right\rangle^{\mathrm{I}} .
$$

The spin $\phi^{2}$ should be interpreted as the overlap of two fermionic excitations. Thus a generic two-excitation eigenstate reads

$$
|\Psi\rangle_{\pi}^{\mathrm{II}}=\left|\psi^{\alpha} \psi^{\beta}\right\rangle_{\pi}^{\mathrm{II}}+C^{\alpha \beta}\left|\phi^{2}\right\rangle_{\pi}^{\mathrm{II}}+\mathcal{P}_{12}^{\mathrm{II}} \mathcal{R}^{\mathrm{II}}\left|\psi^{\alpha} \psi^{\beta}\right\rangle_{\pi}^{\mathrm{II}} .
$$

The matrix $C^{\alpha \beta}$ has the non-zero elements $C^{12}=q^{-1 / 2}$ and $C^{21}=-q^{+1 / 2}$. The operator $\mathcal{P}_{12}^{\mathrm{II}}$ is a (graded) permutation which interchanges the excitations along with their rapidities $y_{1}, y_{2}$. Finally the level-II $R$-matrix $\mathcal{R}^{\text {II }}$ must be invariant under $\mathrm{U}_{q}(\mathfrak{s u}(2))$ which restricts its form to

$$
\begin{aligned}
& \mathcal{R}^{\mathrm{II}}\left|\psi^{1} \psi^{1}\right\rangle^{\mathrm{II}}=M_{12}\left|\psi^{1} \psi^{1}\right\rangle^{\mathrm{II}}, \\
& \mathcal{R}^{\mathrm{II}}\left|\psi^{1} \psi^{2}\right\rangle^{\mathrm{II}}=\frac{M_{12}-N_{12}}{q+q^{-1}}\left|\psi^{1} \psi^{2}\right\rangle^{\mathrm{II}}+\frac{q M_{12}+q^{-1} N_{12}}{q+q^{-1}}\left|\psi^{2} \psi^{1}\right\rangle^{\mathrm{II}}, \\
& \mathcal{R}^{\mathrm{II}}\left|\psi^{2} \psi^{1}\right\rangle^{\mathrm{II}}=\frac{q^{-1} M_{12}+q N_{12}}{q+q^{-1}}\left|\psi^{1} \psi^{2}\right\rangle^{\mathrm{II}}+\frac{M_{12}-N_{12}}{q+q^{-1}}\left|\psi^{2} \psi^{1}\right\rangle^{\mathrm{II}}, \\
& \mathcal{R}^{\mathrm{II}}\left|\psi^{2} \psi^{2}\right\rangle^{\mathrm{II}}=M_{12}\left|\psi^{2} \psi^{2}\right\rangle^{\mathrm{II}} .
\end{aligned}
$$

Imposing the analog of the compatibility condition (4.5) yields a set of equations from which the unknown functions $M_{12}\left(y_{1}, y_{2}\right), N_{12}\left(y_{1}, y_{2}\right), f\left(y_{1}, y_{2}, x_{k}\right)$ can be extracted. The level-II $R$-matrix $\mathcal{R}^{\mathrm{II}, \mathrm{II}}$ can easily be determined by considering any of the triplet of states $\left|\psi^{1} \psi^{1}\right\rangle^{\mathrm{II}}, q^{1 / 2}\left|\psi^{1} \psi^{2}\right\rangle^{\mathrm{II}}+q^{-1 / 2}\left|\psi^{2} \psi^{1}\right\rangle^{\mathrm{II}}$ or $\left|\psi^{2} \psi^{2}\right\rangle^{\mathrm{II}}$. It follows that

$$
M\left(y_{1}, y_{2}\right)=1 \text {. }
$$

To determine the other functions we pick the singlet state $q^{-1 / 2}\left|\psi^{1} \psi^{2}\right\rangle^{\mathrm{II}}-q^{1 / 2}\left|\psi^{2} \psi^{1}\right\rangle^{\mathrm{II}}$. A lengthy calculation shows that

$$
\begin{aligned}
& N\left(y_{1}, y_{2}\right)=\frac{q u\left(y_{2}\right)-q^{-1} u\left(y_{1}\right)+\mathrm{i} g^{-1}}{q^{-1} u\left(y_{2}\right)-q u\left(y_{1}\right)-\mathrm{i} g^{-1}}, \\
& f\left(y_{1}, y_{2}, x_{k}\right)=\frac{\mathrm{i} \alpha\left(q+q^{-1}\right) q^{1 / 2}}{2 g \gamma_{k}^{2}} \frac{x_{k}^{+}\left(x_{k}^{+}-x_{k}^{-}\right)}{\mathrm{i}^{-1}+\left(q-q^{-1}\right) x_{k}^{-}} \frac{y_{1}-y_{2}}{q^{-1} u\left(y_{2}\right)-q u\left(y_{1}\right)-\mathrm{i} g^{-1}} \\
& \cdot\left(s\left(y_{1}\right) s\left(y_{2}\right)-\frac{y_{1} y_{2}}{x_{k}^{+} x_{k}^{-}} \frac{\mathrm{i} g^{-1}+\left(q-q^{-1}\right) x_{k}^{-}}{\mathrm{i}^{-1} y_{1}-\left(q-q^{-1}\right)} \frac{\mathrm{i} g^{-1}+\left(q-q^{-1}\right) x_{k}^{+}}{\mathrm{i}^{-1} y_{2}-\left(q-q^{-1}\right)}\right),
\end{aligned}
$$

is the solution to the compatibility condition if $u(y)$ is the function, see (2.73),

$$
u(y)=y+s(y), \quad s(y)=\frac{\mathrm{i} g^{-1}+\left(q-q^{-1}\right) y}{\mathrm{i} g^{-1} y-\left(q-q^{-1}\right)} .
$$

Note that the level-II $R$-matrix indeed solves the YBE and $u(y)$ is the rapidity variable for this $\mathrm{U}_{q}(\mathfrak{s u}(2)) R$-matrix. The function $u(y)$ is invariant under taking the antipode

$$
u(y)=u(s(y))
$$

because the map $s(y)$ is an involution. 


\subsection{Final level}

The final-level $R$-matrix has the standard form for $\mathrm{U}_{q}(\mathfrak{s u}(2))$ symmetry. The diagonalization by means of the nested Bethe ansatz leads to the following phase factors:

$$
\begin{aligned}
& R^{\mathrm{I}, \mathrm{I}}\left(x_{j}, x_{k}\right)=A\left(x_{j}, x_{k}\right)=R^{0}\left(x_{j}, x_{k}\right) \frac{q^{C_{j}} U_{j}}{q^{C_{k}} U_{k}} \frac{x_{k}^{+}-x_{j}^{-}}{x_{k}^{-}-x_{j}^{+}}, \\
& R^{\mathrm{II}, \mathrm{I}}\left(y_{j}, x_{k}\right)=q^{C_{k}+1 / 2} U_{k} \frac{x_{k}^{-}-y_{j}}{x_{k}^{+}-y_{j}}, \\
& R^{\mathrm{II}, \mathrm{II}}\left(y_{j}, y_{k}\right)=M_{12}\left(y_{j}, y_{k}\right)=1, \\
& R^{\mathrm{III}, \mathrm{II}}\left(w_{j}, y_{k}\right)=q^{-1} \frac{q u\left(y_{k}\right)-w_{j}+\frac{\mathrm{i}}{2} g^{-1}}{q^{-1} u\left(y_{k}\right)-w_{j}-\frac{\mathrm{i}}{2} g^{-1}}, \\
& R^{\mathrm{III}, \mathrm{III}}\left(w_{j}, w_{k}\right)=\frac{q^{-1} w_{k}-q w_{j}-\frac{i}{2}\left(q+q^{-1}\right) g^{-1}}{q w_{k}-q^{-1} w_{j}+\frac{i}{2}\left(q+q^{-1}\right) g^{-1}} .
\end{aligned}
$$

Here $w_{k}$ are the level-III rapidities. For completeness we have included the previously derived phase factors from lower levels.

\subsection{Bethe equations}

Let us now consider a closed spin chain with $K$ sites. The wavefunction for excitations must be periodic in order to define suitable eigenstates. Periodicity is imposed by means of the Bethe equations which use the elements of the diagonalized $R$-matrix. Generically for a nested Bethe ansatz with two levels they can be written as follows:

$$
\begin{aligned}
& 1=\prod_{j=1}^{K} R^{\mathrm{I}, \mathrm{II}}\left(x_{j}, y_{k}\right) \prod_{\substack{j=1 \\
j \neq k}}^{N} R^{\mathrm{II}, \mathrm{II}}\left(y_{j}, y_{k}\right) \prod_{j=1}^{M} R^{\mathrm{III}, \mathrm{II}}\left(w_{j}, y_{k}\right), \\
& 1=\prod_{j=1}^{K} R^{\mathrm{I}, \mathrm{III}}\left(x_{j}, w_{k}\right) \prod_{j=1}^{N} R^{\mathrm{II}, \mathrm{III}}\left(y_{j}, w_{k}\right) \prod_{\substack{j=1 \\
j \neq k}}^{M} R^{\mathrm{III}, \mathrm{III}}\left(w_{j}, y_{k}\right) .
\end{aligned}
$$

Thus for the system in question they take the form

$$
\begin{aligned}
& 1=\prod_{j=1}^{K} q^{-C_{j}-1 / 2} U_{j}^{-1} \frac{y_{k}-x_{j}^{+}}{y_{k}-x_{j}^{-}} \prod_{j=1}^{M} q^{-1} \frac{q u\left(y_{k}\right)-w_{j}+\frac{\mathrm{i}}{2} g^{-1}}{q^{-1} u\left(y_{k}\right)-w_{j}-\frac{\mathrm{i}}{2} g^{-1}} \\
& 1=\prod_{j=1}^{N} q \frac{w_{k}-q^{-1} u\left(y_{j}\right)+\frac{\mathrm{i}}{2} g^{-1}}{w_{k}-q u\left(y_{j}\right)-\frac{\mathrm{i}}{2} g^{-1}} \prod_{\substack{j=1 \\
j \neq k}}^{M} \frac{q^{-1} w_{k}-q w_{j}-\frac{\mathrm{i}}{2}\left(q+q^{-1}\right) g^{-1}}{q w_{k}-q^{-1} w_{j}+\frac{i}{2}\left(q+q^{-1}\right) g^{-1}}
\end{aligned}
$$

\subsection{Dualization}

At the very beginning of the nested Bethe ansatz one can in fact choose between two different vacua, composed from only bosons $\phi^{1}$ 's as above (alternatively $\phi^{2}$ 's) or composed from only fermions $\psi^{1}$ 's (alternatively $\psi^{2}$ 's). In both cases the NBA will proceed in a very similar fashion, but lead to two different but equivalent sets of Bethe equations.

Instead of performing the alternative NBA, we shall derive the alternative Bethe equations by means of dualization. The dualization of our Bethe equations for the undeformed case 
was performed in [18] (which is equivalent to the dualization of the Lieb-Wu equations [3] in [44]). Here the procedure is qualitatively the same, but requires special care due to various insertions of $q$. Let us outline it in the following:

The first Bethe equation in (4.17) can be viewed as an algebraic equation $P\left(y_{k}\right)=0$ with the polynomial

$$
\begin{gathered}
P(y)=\prod_{j=1}^{K} q^{C_{j}+1 / 2} U_{j}\left(y-x_{j}^{-}\right) \prod_{j=1}^{M} q\left(\mathrm{ig}^{-1} y-\left(q-q^{-1}\right)\right)\left(q^{-1} u(y)-w_{j}-\frac{\mathrm{i}}{2} g^{-1}\right) \\
-\prod_{j=1}^{K}\left(y-x_{j}^{+}\right) \prod_{j=1}^{M}\left(\mathrm{i} g^{-1} y-\left(q-q^{-1}\right)\right)\left(q u(y)-w_{j}+\frac{\mathrm{i}}{2} g^{-1}\right) .
\end{gathered}
$$

Note that the common factor $\left(\mathrm{ig}^{-1} y-\left(q-q^{-1}\right)\right)$ was introduced in order to cancel the denominator of $s(y)$ in $u(y)$. This polynomial has degree $K+2 M$, i.e. $N$ of its roots are the $y_{k}$ and the remaining $\tilde{N}=K+2 M-N$ roots will be denoted by $\tilde{y}_{k}$. We can construct a constant function $F(y)$ by dividing by all root monomials

$$
F(y)=P(y) \prod_{j=1}^{N} \frac{1}{y-y_{j}} \prod_{j=1}^{\tilde{N}} \frac{1}{y-\tilde{y}_{j}}=\left(\mathrm{i} g^{-1}\right)^{M}\left(\prod_{j=1}^{K} q^{C_{j}+1 / 2} U_{j}-q^{M}\right)
$$

Next let us define $x_{w}^{ \pm}$as a function of $w_{k}$ implicitly through the relation

$$
w_{k}=q^{-1} u\left(x_{w}^{+}\right)-\frac{\mathrm{i}}{2} g^{-1}=q u\left(x_{w}^{-}\right)+\frac{\mathrm{i}}{2} g^{-1} \text {. }
$$

The $x_{w}^{ \pm}$obey the $x^{ \pm}$-constraint (2.61) which can be written in a particularly convenient form using the map $u(y)(4.13)$,

$$
q^{-1} u\left(x^{+}\right)-q u\left(x^{-}\right)=\frac{\mathrm{i}}{g} .
$$

Now any ratio of the constant function $F(y)$ evaluated for two different values of the parameter equals 1. There are four useful points $y=x_{w}^{ \pm}, s\left(x_{w}^{ \pm}\right)$where one of the two terms in the polynomial $P(y)$ drops out by means of (4.20). At these points we obtain the following identity which holds by virtue of the first Bethe equation in (4.17):

$$
\begin{aligned}
& 1=\frac{F\left(x_{w}^{+}\right) F\left(s\left(x_{w}^{+}\right)\right)}{F\left(x_{w}^{-}\right) F\left(s\left(x_{w}^{-}\right)\right)}=\prod_{j=1}^{K} q^{-2 C_{j}-1} U_{j}^{-2} \frac{x_{w}^{+}-x_{j}^{+}}{x_{w}^{-}-x_{j}^{-}} \frac{s\left(x_{w}^{+}\right)-x_{j}^{+}}{s\left(x_{w}^{-}\right)-x_{j}^{-}} \\
& \cdot \prod_{j=1}^{N} \frac{x_{w}^{-}-y_{j}}{x_{w}^{+}-y_{j}} \frac{s\left(x_{w}^{-}\right)-y_{j}}{s\left(x_{w}^{+}\right)-y_{j}} \prod_{j=1}^{\tilde{N}} \frac{x_{w}^{-}-\tilde{y}_{j}}{x_{w}^{+}-\tilde{y}_{j}} \frac{s\left(x_{w}^{-}\right)-\tilde{y}_{j}}{s\left(x_{w}^{+}\right)-\tilde{y}_{j}} \\
& \cdot\left(q^{-2} \frac{\mathrm{i} g^{-1} x_{w}^{+}-\left(q-q^{-1}\right)}{\mathrm{i} g^{-1} x_{w}^{-}-\left(q-q^{-1}\right)} \frac{\mathrm{ig}^{-1} s\left(x_{w}^{+}\right)-\left(q-q^{-1}\right)}{\mathrm{i}^{-1} s\left(x_{w}^{-}\right)-\left(q-q^{-1}\right)}\right)^{M} \\
& \cdot \prod_{j=1}^{M}\left(\frac{q u\left(x_{w}^{+}\right)-w_{j}+\frac{\mathrm{i}}{2} g^{-1}}{q^{-1} u\left(x_{w}^{-}\right)-w_{j}-\frac{\mathrm{i}}{2} g^{-1}}\right)^{2} \text {. }
\end{aligned}
$$

For the first line we use the identity

$$
\frac{x_{k}^{+}-x_{j}^{+}}{x_{k}^{-}-x_{j}^{-}} \frac{s\left(x_{k}^{+}\right)-x_{j}^{+}}{s\left(x_{k}^{-}\right)-x_{j}^{-}}=q^{2 C_{j}+2} U_{j}^{2}
$$


and obtain simply $q^{K}$. The second line is simplified by means of the identity

$$
\frac{x^{+}-y}{x^{-}-y} \frac{s\left(x^{+}\right)-y}{s\left(x^{-}\right)-y}=\frac{u\left(x^{+}\right)-u(y)}{u\left(x^{-}\right)-u(y)}
$$

and for the third line the identity

$$
\frac{\mathrm{i} g^{-1} x^{+}-\left(q-q^{-1}\right)}{\mathrm{i} g^{-1} x^{-}-\left(q-q^{-1}\right)}=q^{2 C} U^{2}
$$

leads to $q^{-2 M}$. In the last line we substitute the definition (4.20) of $x_{w}^{ \pm}$. Altogether this yields the identity

$$
\begin{gathered}
1=\prod_{j=1}^{N} q^{-1} \frac{w_{k}-q u\left(y_{j}\right)-\frac{\mathrm{i}}{2} g^{-1}}{w_{k}-q^{-1} u\left(y_{j}\right)+\frac{\mathrm{i}}{2} g^{-1}} \prod_{j=1}^{\tilde{N}} q^{-1} \frac{w_{k}-q u\left(\tilde{y}_{j}\right)-\frac{\mathrm{i}}{2} g^{-1}}{w_{k}-q^{-1} u\left(\tilde{y}_{j}\right)+\frac{\mathrm{i}}{2} g^{-1}} \\
\cdot \prod_{\substack{j=1 \\
j \neq k}}^{M}\left(\frac{q w_{k}-q^{-1} w_{j}+\frac{\mathrm{i}}{2}\left(q+q^{-1}\right) g^{-1}}{q^{-1} w_{k}-q w_{j}-\frac{\mathrm{i}}{2}\left(q+q^{-1}\right) g^{-1}}\right)^{2} .
\end{gathered}
$$

Two of these terms coincide with terms in the second Bethe in (4.17). We multiply the Bethe equation and the identity and obtain the dual Bethe equations for $\tilde{y}_{k}$ and $w_{k}$ :

$$
\begin{aligned}
1 & =\prod_{j=1}^{K} q^{C_{j}+1 / 2} U_{j} \frac{\tilde{y}_{k}-x_{j}^{-}}{\tilde{y}_{k}-x_{j}^{+}} \prod_{j=1}^{M} q \frac{q^{-1} u\left(\tilde{y}_{k}\right)-w_{j}-\frac{i}{2} g^{-1}}{q u\left(\tilde{y}_{k}\right)-w_{j}+\frac{\mathrm{i}}{2} g^{-1}}, \\
1 & =\prod_{j=1}^{\tilde{N}} q^{-1} \frac{w_{k}-q u\left(\tilde{y}_{j}\right)-\frac{\mathrm{i}}{2} g^{-1}}{w_{k}-q^{-1} u\left(\tilde{y}_{j}\right)+\frac{\mathrm{i}}{2} g^{-1}} \prod_{\substack{j=1 \\
j \neq k}}^{M} \frac{q w_{k}-q^{-1} w_{j}+\frac{\mathrm{i}}{2}\left(q+q^{-1}\right) g^{-1}}{q^{-1} w_{k}-q w_{j}-\frac{\mathrm{i}}{2}\left(q+q^{-1}\right) g^{-1}} .
\end{aligned}
$$

These Bethe equations do not contain the scattering term $R^{\mathrm{I}, \mathrm{I}}\left(x_{j}, x_{k}\right)$ which should be dualized as well for completeness. In fact it can easily be obtained from the scattering in the dual level-II vacuum composed from $\psi^{1}$ 's: it equals $-D\left(x_{j}, x_{k}\right)$. An alternative procedure is to consider the $x_{k}^{ \pm}$as dynamical degrees of freedom as in [18]. In other words they obey some Bethe equation of the form

$$
\begin{aligned}
\ldots & =\prod_{\substack{j=1 \\
j \neq k}}^{K} R^{\mathrm{I}, \mathrm{I}}\left(x_{j}, x_{k}\right) \prod_{j=1}^{N} R^{\mathrm{II}, \mathrm{I}}\left(y_{j}, x_{k}\right) \prod_{j=1}^{M} R^{\mathrm{III}, \mathrm{I}}\left(w_{j}, x_{k}\right) \\
& =\prod_{\substack{j=1 \\
j \neq k}}^{K} R^{0}\left(x_{j}, x_{k}\right) \frac{q^{C_{j}} U_{j}}{q^{C_{k}} U_{k}} \frac{x_{k}^{+}-x_{j}^{-}}{x_{k}^{-}-x_{j}^{+}} \prod_{j=1}^{N} q^{C_{k}+1 / 2} U_{k} \frac{x_{k}^{-}-y_{j}}{x_{k}^{+}-y_{j}} .
\end{aligned}
$$

We can now derive another identity from the constant function $F(y)$ :

$$
\begin{aligned}
1=\frac{F\left(x_{k}^{-}\right)}{F\left(x_{k}^{+}\right)}= & -\prod_{j=1}^{N} \frac{x_{k}^{+}-y_{j}}{x_{k}^{-}-y_{j}} \prod_{j=1}^{\tilde{N}} \frac{x_{k}^{+}-\tilde{y}_{j}}{x_{k}^{-}-\tilde{y}_{j}} \prod_{j=1}^{K} q^{-C_{j}-1 / 2} U_{j}^{-1} \frac{x_{k}^{-}-x_{j}^{+}}{x_{k}^{+}-x_{j}^{-}} \\
& \cdot\left(q \frac{\mathrm{i} g^{-1} x_{k}^{+}-\left(q-q^{-1}\right)}{\mathrm{i} g^{-1} x_{k}^{-}-\left(q-q^{-1}\right)}\right)^{-M} \prod_{j=1}^{M} \frac{q u\left(x_{k}^{-}\right)-w_{j}+\frac{\mathrm{i}}{2} g^{-1}}{q^{-1} u\left(x_{k}^{+}\right)-w_{j}-\frac{\mathrm{i}}{2} g^{-1}} .
\end{aligned}
$$


Using the identities (4.21)-(4.25) we find

$1=\prod_{j=1}^{N} q^{-C_{k}-1 / 2} U_{k}^{-1} \frac{x_{k}^{+}-y_{j}}{x_{k}^{-}-y_{j}} \prod_{j=1}^{\tilde{N}} q^{-C_{k}-1 / 2} U_{k}^{-1} \frac{x_{k}^{+}-\tilde{y}_{j}}{x_{k}^{-}-\tilde{y}_{j}} \prod_{\substack{j=1 \\ j \neq k}}^{K} \frac{q^{C_{k}} U_{k}}{q^{C_{j}} U_{j}} \frac{x_{k}^{-}-x_{j}^{+}}{x_{k}^{+}-x_{j}^{-}}$,

which yields when multiplied to (4.28) the dual Bethe equation

$$
\cdots=\prod_{\substack{j=1 \\ j \neq k}}^{K} R^{0}\left(x_{j}, x_{k}\right) \prod_{j=1}^{\tilde{N}} q^{-C_{k}-1 / 2} U_{k}^{-1} \frac{x_{k}^{+}-\tilde{y}_{j}}{x_{k}^{-}-\tilde{y}_{j}} .
$$

In conclusion the dual phase factors read

$$
\begin{aligned}
& \tilde{R}^{\mathrm{I}, \mathrm{I}}\left(x_{j}, x_{k}\right)=-D\left(x_{j}, x_{k}\right)=R^{0}\left(x_{j}, x_{k}\right), \\
& \tilde{R}^{\mathrm{II}, \mathrm{I}}\left(\tilde{y}_{j}, x_{k}\right)=q^{-C_{k}-1 / 2} U_{k}^{-1} \frac{x_{k}^{+}-\tilde{y}_{j}}{x_{k}^{-}-\tilde{y}_{j}}, \\
& \tilde{R}^{\mathrm{II}, \mathrm{II}}\left(\tilde{y}_{j}, \tilde{y}_{k}\right)=1, \\
& \tilde{R}^{\mathrm{III}, \mathrm{II}}\left(w_{j}, \tilde{y}_{k}\right)=q \frac{q^{-1} u\left(\tilde{y}_{k}\right)-w_{j}-\frac{\mathrm{i}}{2} g^{-1}}{q u\left(\tilde{y}_{k}\right)-w_{j}+\frac{\mathrm{i}}{2} g^{-1}}, \\
& \tilde{R}^{\mathrm{III}, \mathrm{III}}\left(w_{j}, w_{k}\right)=\frac{q w_{k}-q^{-1} w_{j}+\frac{\mathrm{i}}{2}\left(q+q^{-1}\right) g^{-1}}{q^{-1} w_{k}-q w_{j}-\frac{\mathrm{i}}{2}\left(q+q^{-1}\right) g^{-1}} .
\end{aligned}
$$

\section{Quantum deformation of the Hubbard model}

In this section we derive an integrable homogeneous nearest-neighbor Hamiltonian from the $\mathrm{U}_{q}(\mathfrak{h}) R$-matrix in table 1. Its Hilbert space is the same as for the one-dimensional Hubbard model and we will show that our Hamiltonian is a three-parameter deformation of the Hubbard Hamiltonian. We then discuss the relation to the integrable two-parameter deformations of the Hubbard model proposed by Alcaraz and Bariev [34] and introduce additional sets of two-parameter deformations.

\subsection{Hamiltonian}

In the following we derive a nearest-neighbor spin-chain Hamiltonian from the $\mathrm{U}_{q}(\mathfrak{h}) R$-matrix in table 1.

Integrable Hamiltonian. For an integrable spin chain based on an $R$-matrix there is a standard procedure to obtain a homogeneous Hamiltonian

$$
\mathcal{H}=\sum_{k=1}^{L} \mathcal{H}_{k, k+1}
$$

The pairwise interaction $\mathcal{H}_{12}$ is the following logarithmic derivative of the $R$-matrix:

$$
\mathcal{H}_{12}=-\left.\mathrm{i} \frac{\left(x^{+}-s\left(x^{+}\right)\right)\left(x^{-}-s\left(x^{-}\right)\right)}{q^{-1} x^{+} s\left(x^{+}\right)}\left(\frac{\mathrm{d} u^{*}}{\mathrm{~d} u}\right)^{-1 / 2} \mathcal{R}_{12}^{-1} \frac{\mathrm{d}}{\mathrm{d} u_{1}} \mathcal{R}_{12}\right|_{x_{12}^{ \pm}=x^{ \pm}} .
$$

The spectral parameters $u_{k}$ are defined as functions of the parameters $x_{k}^{ \pm}$, see (4.13),

$$
u_{k}=q^{-1} u\left(x_{k}^{+}\right)-\frac{\mathrm{i}}{2 g}=q u\left(x_{k}^{-}\right)+\frac{\mathrm{i}}{2 g} \text {. }
$$


Table 3. General form of the nearest-neighbor $\mathrm{U}_{q}(\mathfrak{s u}(2) \times \mathfrak{s u}(2))$ spin-chain Hamiltonian.

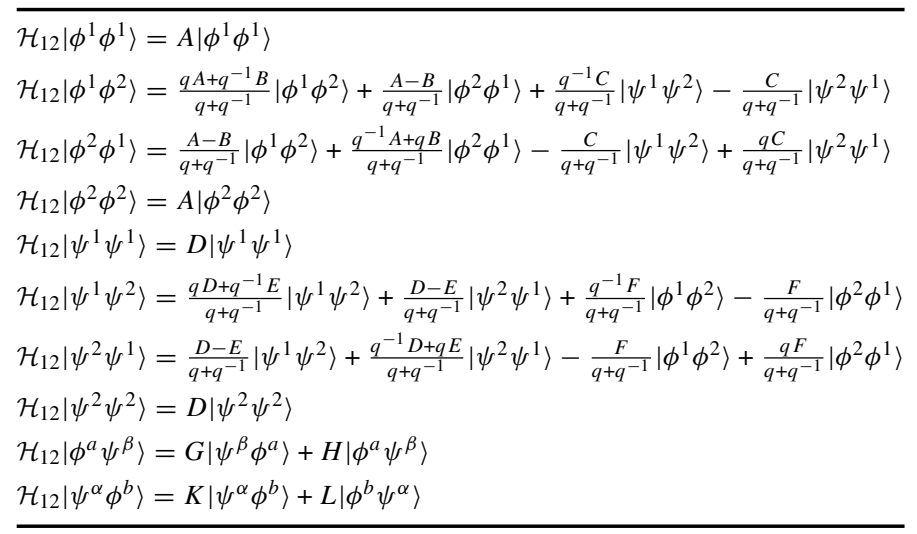

Table 4. The coefficients for the nearest-neighbor Hamiltonian.

$A=-D=\frac{1}{4 g} \frac{\left(q^{C} U+q^{-C} U^{-1}\right)\left(q^{C} U^{-1}+q^{-C} U\right)}{\left(q^{C} U-q^{-C} U^{-1}\right)\left(q^{C} U^{-1}-q^{-C} U\right)}$
$A-B=E-D=\frac{q+q^{-1}}{g} \frac{1}{\left(q^{C} U-q^{-C} U^{-1}\right)\left(q^{C} U^{-1}-q^{-C} U\right)}$
$C=F=\left(q+q^{-1}\right) \sqrt{1-\left(q-q^{-1}\right)^{2} g^{2}}$
$G=\frac{q^{C+1 / 2} U^{-1}-q^{-C-1 / 2} U^{-1}-q^{C-1 / 2} U+q^{-C+1 / 2} U}{g\left(q-q^{-1}\right)\left(q^{C} U-q^{-C} U^{-1}\right)\left(q^{C} U^{-1}-q^{-C} U\right)}$
$L=\frac{q^{C+1 / 2} U-q^{-C-1 / 2} U-q^{C-1 / 2} U^{-1}+q^{-C+1 / 2} U^{-1}}{g\left(q-q^{-1}\right)\left(q^{C} U-q^{-C} U^{-1}\right)\left(q^{C} U^{-1}-q^{-C} U\right)}$
$H=K=0$

Note that $u_{k}$ is not real

$u_{k}^{*}=\frac{\left(q+q^{-1}\right) u_{k}+\frac{\mathrm{i}}{2} g^{-1}\left(q-q^{-1}\right)}{\left(q+q^{-1}\right)-2 \mathrm{i} g\left(q-q^{-1}\right) u_{k}}, \quad \frac{\mathrm{d} u_{k}^{*}}{\mathrm{~d} u_{k}}=\left(q^{-1} x_{k}^{+} s\left(x_{k}^{+}\right)\right)^{-2}=\left(q x_{k}^{-} s\left(x_{k}^{-}\right)\right)^{-2}$,

and therefore we have included a compensating prefactor $\left(\mathrm{d} u^{*} / \mathrm{d} u\right)^{-1 / 2}=q^{-1} x_{k}^{+} s\left(x_{k}^{+}\right)$to make the Hamiltonian Hermitian. The additional real prefactor is meant to simplify the resulting expressions.

The resulting Hamiltonian clearly has $\mathrm{U}_{q}(\mathfrak{s u}(2) \times \mathfrak{s u}(2))$ symmetry ${ }^{9}$ and the general form with such symmetry is listed in table 3 . Just like the $R$-matrix in table 1 is has ten independent coefficients $X=A, B, C, D, E, F, G, H, K, L$. We have arranged them such that they are directly related to the corresponding coefficients $X_{12}$ of the $R$-matrix in table 2 according to

$$
\begin{aligned}
X & =\left.\mathrm{i}\left(x^{+}-s\left(x^{+}\right)\right)\left(x^{-}-s\left(x^{-}\right)\right) \frac{\mathrm{d} X_{12}}{\mathrm{~d} u_{1}}\right|_{x_{1,2}^{ \pm}=x^{ \pm}} \\
& =\left.\mathrm{i}\left(x^{+}-s\left(x^{+}\right)\right)\left(x^{-}-s\left(x^{-}\right)\right)\left(\frac{\mathrm{d} x^{+}}{\mathrm{d} u} \frac{\partial X_{12}}{\partial x_{1}^{+}}+\frac{\mathrm{d} x^{-}}{\mathrm{d} u} \frac{\partial X_{12}}{\partial x_{1}^{-}}\right)\right|_{x_{1,2}^{ \pm}=x^{ \pm}} .
\end{aligned}
$$

9 It does not necessarily have $\mathrm{U}_{q}(\mathfrak{h})$ symmetry due to the $u$-dependence of the coefficients $a, b, c, d$ in the representation of the fermionic generators. In fact there is no $\mathrm{U}_{q}(\mathfrak{h})$-invariant operator apart from the identity for generic $q$. 
Note the following two useful identities in evaluating the derivatives:

$$
\begin{aligned}
& \frac{\mathrm{d} x^{ \pm}}{\mathrm{d} u}=\frac{x^{+}-x^{-}}{x^{ \pm}-s\left(x^{ \pm}\right)} \frac{q^{ \pm C \pm 1} U^{ \pm 1}}{q^{C} U-q^{-C} U^{-1}} \\
& \frac{\mathrm{d}}{\mathrm{d} u} \log \left(U q^{C}\right)=\frac{1}{2}\left(\frac{q}{x^{+}-s\left(x^{+}\right)}-\frac{q^{-1}}{x^{-}-s\left(x^{-}\right)}\right) .
\end{aligned}
$$

The coefficients $X$ still depend on the parameters $\alpha, \gamma_{k}$ and the phase factor $R_{12}^{0}$. The latter two can furthermore depend non-trivially on $x_{k}^{ \pm}$which would influence the Hamiltonian explicitly or implicitly through the derivatives. For definiteness we set the phase factor to

$$
R_{12}^{0}=\sqrt{\frac{q^{C_{2}} U_{2}}{q^{C_{1}} U_{1}} \frac{x_{2}^{-}-x_{1}^{+}}{x_{2}^{+}-x_{1}^{-}}} ;
$$

a different phase factor would induce an overall shift of the spectrum which we shall incorporate explicitly later. The most suitable expression for $\gamma_{k}$ is given in (2.65) and we shall set the global parameter $\alpha$ to unity, $\alpha=1$.

Now we are ready to obtain an explicit expression for the nearest-neighbor Hamiltonian. Taking into account that for $x_{1}^{ \pm}=x_{2}^{ \pm}$the $R$-matrix becomes minus the graded permutation operator one can show that the Hamiltonian has the coefficients listed in table 4.

Integrability constraints. The coefficients obey certain relations: there are two linear relations and two quadratic relations:

$$
\begin{aligned}
& A+D=B+E=H+K, \\
& (A-B)^{2}+C F=\left(q+q^{-1}\right)^{2} G L, \\
& 2(A-B)(A-D)=\left(q+q^{-1}\right)\left(G^{2}+\left(q+q^{-1}\right) G L+L^{2}\right) .
\end{aligned}
$$

Note that the two linear relations can be derived from the identity (3.6); the origin of the two quadratic relations remains unclear. These equations are invariant under four trivial transformations: ( i) A rescaling of all coefficients by a common factor. (ii) A shift of the two-site Hamiltonian by the two-site identity operator $\Delta(1)$, taking the form in table 3 with

$$
A=B=D=E=H=K=1, \quad C=F=G=L=0 .
$$

(iii) A reciprocal rescaling of $C$ and $F$ corresponding to a different rescaling of fermions $\left|\psi^{\alpha}\right\rangle$ with respect to bosons $\left|\phi^{a}\right\rangle$. (iv) An opposite shift of $H$ and $K$ which has no impact on the spectrum. Altogether the ten coefficients together with the parameter $q$ subject to the four constraints (5.8) and four identifications $(i-i v)$ define a three-parameter family of models. This is the same number of degrees of freedom as for the fundamental $R$-matrix given in terms of $q, g, x^{ \pm}$subject to the one constraint (2.61). Therefore the constraints (5.8) are expected to be sufficient to ensure integrability of the Hamiltonian in table 3.

Hermiticity. There are ten independent complex coefficients in the Hamiltonian in table 3. The Hermiticity condition $\mathcal{H}_{12}^{\dagger}=\mathcal{H}_{12}$ imposes certain reality conditions on the coefficients

$$
C^{*}=F, \quad G^{*}=L, \quad A, B, D, E, H, K \in \mathbb{R} .
$$

Furthermore we have to require that ${ }^{10}$

$$
q \in \mathbb{R} \text {. }
$$

Due to our choice (2.65) for $\gamma_{k}$, we have that $C=F$ and thus $C, F$ must both be real.

${ }^{10}$ Usually in quantum-deformed spin chains the case of $q$ on the unit circle also leads to Hermitian Hamiltonians. Here we have not been managed to establish Hermiticity in this case. 
Generalizations. Before performing an explicit comparison of the models in question we would like to introduce certain integrable generalizations of the Hamiltonian which change the spectrum in a controllable fashion. Thus given a Hamiltonian $\mathcal{H}_{12}$ as in table 3 one can transform it as follows:

$$
\begin{gathered}
\mathcal{H}_{12}^{\prime}=a_{0} \mathcal{T H}_{12} \mathcal{T}^{-1}+\frac{1}{2} a_{1} \Delta\left(\mathfrak{H}_{1}\right)+a_{2} \Delta(1)+\frac{1}{2} a_{3} \Delta\left(\mathfrak{H}_{3}\right)+\frac{1}{2} b_{1}\left(\mathfrak{H}_{1} \otimes 1-1 \otimes \mathfrak{H}_{1}\right) \\
+b_{2}\left(\mathfrak{H}_{1} \mathfrak{H}_{1} \otimes 1-1 \otimes \mathfrak{H}_{1} \mathfrak{H}_{1}\right)+\frac{1}{2} b_{3}\left(\mathfrak{H}_{3} \otimes 1-1 \otimes \mathfrak{H}_{3}\right)
\end{gathered}
$$

Here $a_{k}, b_{k}$ are arbitrary constants. Indeed $a_{0}$ is an overall multiplier of the operator, $a_{2}$ is an overall shift and $a_{1}, a_{3}$ correspond to a shift of the energy eigenvalues proportional to the $\mathfrak{H}_{1}, \mathfrak{H}_{3}$ Cartan generator eigenvalues. The terms multiplied by $b_{k}$ vanish after summation over the whole spin chain with periodic boundary conditions (5.1).

The similarity transformation induced by $\mathcal{T}$ is the following Reshetikhin twist [45] of the integrable structure

$$
\Delta^{\prime}(\mathfrak{J})=\mathcal{T} \Delta(\mathfrak{J}) \mathcal{T}^{-1}, \quad \mathcal{R}^{\prime}=\mathcal{P}(\mathcal{T}) \mathcal{R} \mathcal{T}^{-1}
$$

It is not hard to verify that the twist preserves the cocommutativity property. We shall consider a twist $\mathcal{T}$ constructed from the identity and Cartan generators

$$
\mathcal{T}=\exp \left(i f_{1}\left(1 \otimes \mathfrak{H}_{1}\right)+\frac{\mathrm{i}}{2} f_{2}\left(\mathfrak{H}_{1} \otimes \mathfrak{H}_{3}-\mathfrak{H}_{3} \otimes \mathfrak{H}_{1}\right)+\mathrm{i} f_{3}\left(1 \otimes \mathfrak{H}_{3}\right)\right)
$$

which can be generalized consistently to arbitrarily many sites

$\mathcal{T}=\exp \left(\mathrm{i} f_{1} \sum_{j=1}^{K}(j-1) \mathfrak{H}_{1, j}+\frac{\mathrm{i}}{2} f_{2} \sum_{j<k=1}^{K}\left(\mathfrak{H}_{1, j} \mathfrak{H}_{3, k}-\mathfrak{H}_{3, j} \mathfrak{H}_{1, k}\right)+\mathrm{i} f_{3} \sum_{j=1}^{K}(j-1) \mathfrak{H}_{3, k}\right)$.

The coefficients $f_{k}$ are arbitrary parameters. Hermiticity requires them to be real.

\subsection{Bethe equations and spectrum}

The spectrum of the above Hamiltonian on a closed homogeneous spin chain is determined by Bethe equations. Here we specify the Bethe equations and energy relations.

Energies. We use the Bethe ansatz based on a ferromagnetic vacuum consisting of $K$ spins $\phi^{1}$ as in (4.1). We assume there are $N$ main excitations (magnons) with momenta $p_{k}$ which turn a $\phi^{1}$ into a $\psi^{1}$. Finally, there are $M$ auxiliary excitations with rapidities $w_{k}$ which turn a $\psi^{1}$ into a $\psi^{2}$. We have shown in section 4 that the Bethe equations for this system are given by (4.17) where we set all representation parameters to be equal $x_{k}^{ \pm}=x^{ \pm}$for a homogeneous chain

$$
\begin{aligned}
& 1=\left(q^{-C-1 / 2} U^{-1} \frac{y_{k}-x^{+}}{y_{k}-x^{-}}\right)^{K} \prod_{j=1}^{M} q^{-1} \frac{q u\left(y_{k}\right)-w_{j}+\frac{\mathrm{i}}{2} g^{-1}}{q^{-1} u\left(y_{k}\right)-w_{j}-\frac{\mathrm{i}}{2} g^{-1}}, \\
& 1=\prod_{j=1}^{N} q \frac{w_{k}-q^{-1} u\left(y_{j}\right)+\frac{\mathrm{i}}{2} g^{-1}}{w_{k}-q u\left(y_{j}\right)-\frac{\mathrm{i}}{2} g^{-1}} \prod_{\substack{j=1 \\
j \neq k}}^{M} \frac{q^{-1} w_{k}-q w_{j}-\frac{\mathrm{i}}{2}\left(q+q^{-1}\right) g^{-1}}{q w_{k}-q^{-1} w_{j}+\frac{\mathrm{i}}{2}\left(q+q^{-1}\right) g^{-1}} .
\end{aligned}
$$

First of all, a relation between the magnon momenta $p_{k}$ and the magnon rapidities $y_{k}$ has to be established. In the Bethe equations (4.16) the term $R^{\mathrm{I}, \mathrm{II}}\left(x, y_{k}\right)$ serves the purpose of $\mathrm{e}^{-\mathrm{i} p_{k}}$, i.e. the rapidity relation is

$$
\mathrm{e}^{\mathrm{i} p_{k}}=R^{\mathrm{II}, \mathrm{I}}\left(y_{k}, x\right)=q^{C+1 / 2} U \frac{y_{k}-x^{-}}{y_{k}-x^{+}}, \quad \mathrm{e}^{\mathrm{i} P}=\prod_{j=1}^{N} \mathrm{e}^{\mathrm{i} p_{k}} .
$$


Here the total spin-chain momentum is given by $P$. The energy for a solution of the Bethe equations is given as the sum of vacuum energy and magnon energies (here $K$ refers to the length of the chain)

$$
E=E_{0} K+\sum_{j=1}^{N} E\left(y_{j}\right)
$$

The vacuum energy density $E_{0}$ and magnon dispersion relation $E\left(y_{k}\right)$ follow readily from the expression for the Hamiltonian in table 3 (here $K$ refers to the coefficient $K$ of the Hamiltonian)

$$
E_{0}=A, \quad E\left(y_{k}\right)=H+K-2 A+G \mathrm{e}^{\mathrm{i} p_{k}}+L \mathrm{e}^{-\mathrm{i} p_{k}},
$$

where $\mathrm{e}^{\mathrm{i} p_{k}}$ is defined in (5.17) as a function of $y_{k}$. The energy relation can also be obtained formally from the diagonalized elements of the $R$-matrix in analogy to (5.17):

$$
\begin{aligned}
& E_{0}=\left.\mathrm{i}\left(x^{+}-s\left(x^{+}\right)\right)\left(x^{-}-s\left(x^{-}\right)\right) \frac{\mathrm{d}}{\mathrm{d} u} \log R^{\mathrm{I}, \mathrm{I}}\left(x_{0}, x\right)\right|_{x_{0}^{ \pm}=x^{ \pm}}, \\
& E\left(y_{k}\right)=\mathrm{i}\left(x^{+}-s\left(x^{+}\right)\right)\left(x^{-}-s\left(x^{-}\right)\right) \frac{\mathrm{d}}{\mathrm{d} u} \log R^{\mathrm{II}, \mathrm{I}}\left(y_{k}, x\right) .
\end{aligned}
$$

This leads to the same as the above expressions.

Transformation. The above generalization of the Hamiltonian (5.12) requires certain modifications of the just derived expressions. Most importantly, the Bethe equations receive further phase factors due to the twist induced by the $f_{k}^{11}$

$$
\begin{aligned}
& 1=\left(\mathrm{e}^{\mathrm{i}\left(f_{3}-f_{1}-f_{2}\right)} q^{-C-1 / 2} U^{-1} \frac{y_{k}-x^{+}}{y_{k}-x^{-}}\right) \prod_{j=1}^{K} \mathrm{e}^{2 \mathrm{i} f_{2}} q^{-1} \frac{q u\left(y_{k}\right)-w_{j}+\frac{\mathrm{i}}{2} g^{-1}}{q^{-1} u\left(y_{k}\right)-w_{j}-\frac{\mathrm{i}}{2} g^{-1}}, \\
& 1=\mathrm{e}^{2 \mathrm{i}\left(f_{2}-f_{3}\right) K} \prod_{j=1}^{N} \mathrm{e}^{-2 \mathrm{i} f_{2}} q \frac{w_{k}-q^{-1} u\left(y_{j}\right)+\frac{\mathrm{i}}{2} g^{-1}}{w_{k}-q u\left(y_{j}\right)-\frac{\mathrm{i}}{2} g^{-1}} \prod_{\substack{j=1 \\
j \neq k}}^{M} \frac{q^{-1} w_{k}-q w_{j}-\frac{\mathrm{i}}{2}\left(q+q^{-1}\right) g^{-1}}{q w_{k}-q^{-1} w_{j}+\frac{\mathrm{i}}{2}\left(q+q^{-1}\right) g^{-1}} .
\end{aligned}
$$

These phase factors therefore enter the momentum relation $\mathrm{e}^{\mathrm{i} p_{k}}=R^{\mathrm{II}, \mathrm{I}}\left(y_{k}, x\right)$ as follows:

$$
\mathrm{e}^{\mathrm{i} p_{k}}=\mathrm{e}^{\mathrm{i}\left(f_{1}+f_{2}-f_{3}\right)} q^{C+1 / 2} U \frac{y_{k}-x^{-}}{y_{k}-x^{+}} .
$$

Due to the logarithmic derivatives in (5.20) the expression for the energy is unaffected by the phase factors. It however receives contributions from the rescaling and shifts in (5.12)

$$
E^{\prime}=\left(a_{0} E_{0}-a_{1}+a_{2}\right) K+2 a_{3} M+\sum_{j=1}^{N}\left(a_{0} E\left(y_{j}\right)+a_{1}-a_{3}\right)
$$

Dual Picture. The procedure to obtain the spectrum in the dual picture discussed in section 4.5 is the same. Here the ferromagnetic vacuum is based on $\psi^{1}$ 's, the magnons flip them to $\phi^{1}$ 's and auxiliary excitations flip the latter to $\phi^{2}$ 's. The twisted version of the dual Bethe equations in (4.27) has the following insertions of phase factors:

$$
\begin{aligned}
& 1=\left(\mathrm{e}^{\mathrm{i}\left(f_{1}+f_{2}-f_{3}\right)} q^{C+1 / 2} U \frac{\tilde{y}_{k}-x^{-}}{\tilde{y}_{k}-x_{j}^{+}}\right)^{K} \prod_{j=1}^{M} \mathrm{e}^{-2 \mathrm{i} f_{2}} q \frac{q^{-1} u\left(\tilde{y}_{k}\right)-w_{j}-\frac{\mathrm{i}}{2} g^{-1}}{q u\left(\tilde{y}_{k}\right)-w_{j}+\frac{\mathrm{i}}{2} g^{-1}}, \\
& 1=\mathrm{e}^{2 \mathrm{i}\left(-f_{1}-f_{2}\right) K} \prod_{j=1}^{\tilde{N}} \mathrm{e}^{2 \mathrm{i} f_{2}} q^{-1} \frac{w_{k}-q u\left(\tilde{y}_{j}\right)-\frac{\mathrm{i}}{2} g^{-1}}{w_{k}-q^{-1} u\left(\tilde{y}_{j}\right)+\frac{\mathrm{i}}{2} g^{-1}} \prod_{\substack{j=1 \\
j \neq k}}^{M} \frac{q w_{k}-q^{-1} w_{j}+\frac{\mathrm{i}}{2}\left(q+q^{-1}\right) g^{-1}}{q^{-1} w_{k}-q w_{j}-\frac{\mathrm{i}}{2}\left(q+q^{-1}\right) g^{-1}} .
\end{aligned}
$$

${ }^{11}$ Clearly the twist can also be applied to the inhomogeneous equations where $x_{k}^{ \pm} \neq x_{j}^{ \pm}$. 
To obtain the correct momentum relation we have to remember that the vacuum as well as the magnons are fermionic excitations. Therefore there is an implicit factor of -1 in the momentum relation $\mathrm{e}^{\mathrm{i} \tilde{p}_{k}}=-\tilde{R}^{\mathrm{II}, \mathrm{I}}\left(\tilde{y}_{k}, x\right)$ :

$$
\mathrm{e}^{\mathrm{i} \tilde{p}_{k}}=-\mathrm{e}^{\mathrm{i}\left(f_{3}-f_{1}-f_{2}\right)} q^{-C-1 / 2} U^{-1} \frac{\tilde{y}_{k}-x^{+}}{\tilde{y}_{k}-x^{-}}, \quad \mathrm{e}^{\mathrm{i} P}=(-1)^{K+\tilde{N}-1} \prod_{j=1}^{\tilde{N}} \mathrm{e}^{\mathrm{i} \tilde{p}_{j}} .
$$

Note that the definition of the total momentum $P$ via the cyclic shift operator requires taking into account the statistics of the vacuum sites and excitations. The energy relation uses the above dispersion relation $E(y)$ up to a sign

$$
E^{\prime}=\left(-a_{0} E_{0}-a_{3}+a_{2}\right) K+2 a_{1} M+\sum_{j=1}^{\tilde{N}}\left(-a_{0} E\left(\tilde{y}_{j}\right)-a_{1}+a_{3}\right)
$$

The equality of the total momentum $P$ and total energy $E^{\prime}$ in both pictures makes use of the identity (4.30) which guarantees for all eigenstates that

$$
\prod_{j=1}^{N} \mathrm{e}^{\mathrm{i} p_{j}} \prod_{j=1}^{\tilde{N}} \mathrm{e}^{-\mathrm{i} \tilde{p}_{j}}=(-1)^{K+\tilde{N}-1}, \quad \sum_{j=1}^{N} E\left(y_{j}\right)+\sum_{j=1}^{\tilde{N}} E\left(\tilde{y}_{j}\right)=-2 E_{0} K .
$$

\subsection{Electronic oscillator notation}

We have established the Hamiltonian in terms of a matrix acting on a spin chain based on four-dimensional vector spaces. Let us rewrite the four-dimensional vector space in terms of fermionic 'electron' creation and annihilation operators $c_{\alpha}^{\dagger}$ and $c_{\alpha}$ as is usually done in the Hubbard model literature.

The map between vector states and electronic states reads ${ }^{12}$

$$
\left|\phi^{1}\right\rangle=|\circ\rangle, \quad\left|\phi^{2}\right\rangle=\kappa c_{1}^{\dagger} c_{2}^{\dagger}|\circ\rangle, \quad\left|\psi^{1}\right\rangle=c_{1}^{\dagger}|\circ\rangle, \quad\left|\psi^{2}\right\rangle=c_{2}^{\dagger}|\circ\rangle .
$$

where creation and annihilation operators satisfy the following algebra

$$
\left\{c_{\alpha}, c_{\beta}^{\dagger}\right\}=\delta_{\alpha \beta}, \quad\left\{c_{\alpha}, c_{\beta}\right\}=\left\{c_{\alpha}^{\dagger}, c_{\beta}^{\dagger}\right\}=0 .
$$

We define the number operator by

$$
n_{\alpha}=c_{\alpha}^{\dagger} c_{\alpha}
$$

Note that $n_{\alpha}^{2}=n_{\alpha}$, hence $n_{\alpha}$ is a projector onto the subspace spanned by $\left|\psi^{\alpha}\right\rangle,\left|\phi^{2}\right\rangle$. The following projectors can be specified:

$$
\begin{aligned}
& \left|\phi^{1}\right\rangle\left\langle\phi_{1}\right|=\left(1-n_{1}\right)\left(1-n_{2}\right), \\
& \left|\phi^{2}\right\rangle\left\langle\phi_{2}\right|=n_{1} n_{2} \\
& \left|\psi^{1}\right\rangle\left\langle\psi_{1}\right|=n_{1}\left(1-n_{2}\right) \\
& \left|\psi^{2}\right\rangle\left\langle\psi_{2}\right|=n_{2}\left(1-n_{1}\right)
\end{aligned}
$$

From these projectors many useful combinations can be assembled straightforwardly. The Cartan generators can be rewritten in terms of number operators as follows

$$
\mathfrak{H}_{1}=n_{1}+n_{2}-1, \quad \mathfrak{H}_{3}=n_{2}-n_{1} .
$$

${ }^{12}$ More generally, we may introduce different normalization factors for the four states. By rescaling the state $|0\rangle$ and the generators $c_{1}^{\dagger}, c_{2}^{\dagger}$ these can all be absorbed into a single variable $\kappa$. 
Table 5. Oscillator form of the $\mathrm{U}_{q}(\mathfrak{s u}(2) \times \mathfrak{s u}(2))$ spin-chain Hamiltonian.

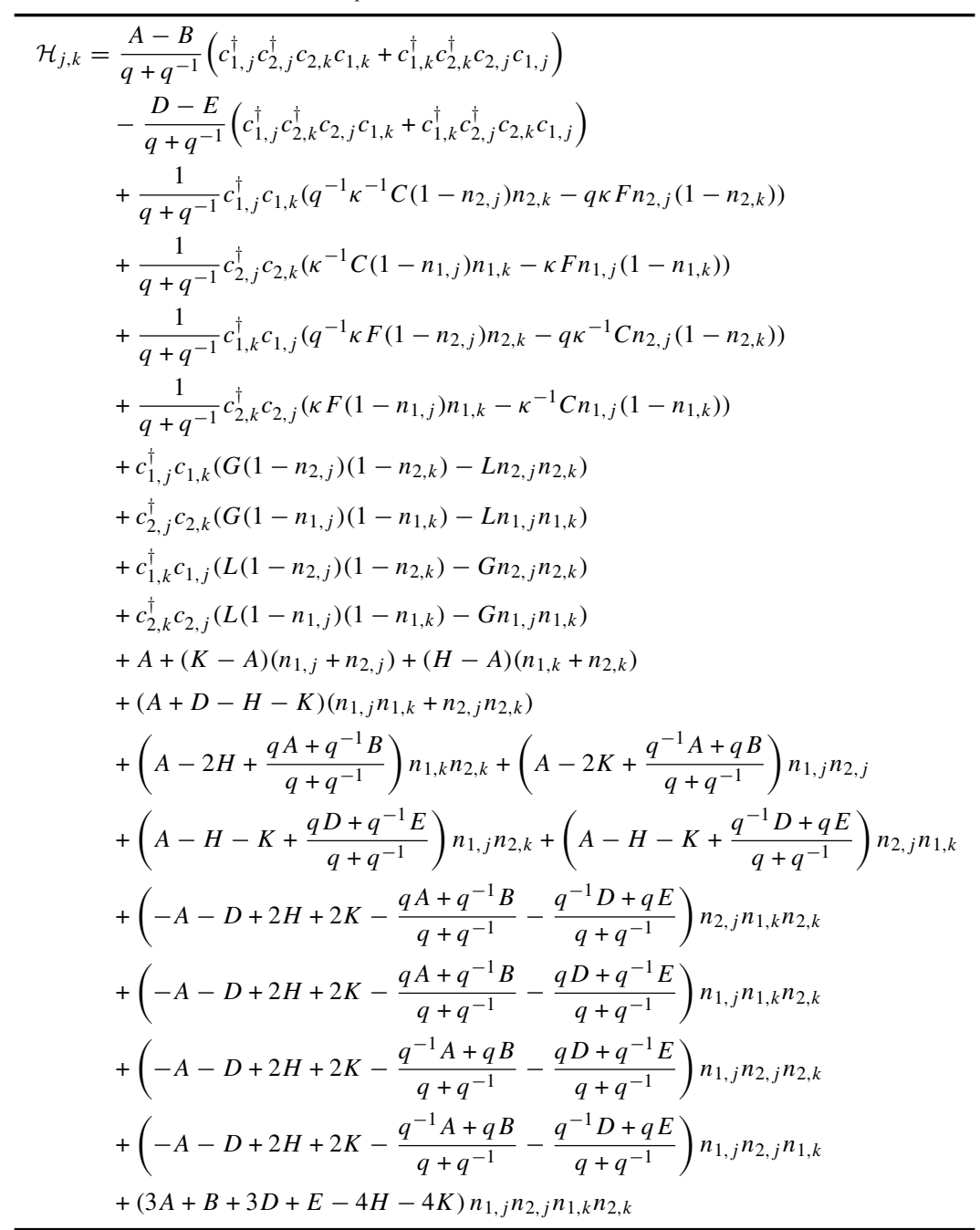

For dealing with spin chains we introduce multi-site oscillators by adding a site index $j, k$. The oscillator algebra becomes

$$
\left\{c_{\alpha, j}, c_{\beta, k}^{\dagger}\right\}=\delta_{j k} \delta_{\alpha \beta}, \quad\left\{c_{\alpha, j}, c_{\beta, k}\right\}=\left\{c_{\alpha, j}^{\dagger}, c_{\beta, k}^{\dagger}\right\}=0 .
$$

By means of the projectors specified above we can spell out the Hamiltonian of our model in oscillator notation. Thus for the operator from table 3 we have the expression listed in table 5 .

\subsection{Connection with the one-dimensional Hubbard model}

In two special limits the spin chain with undeformed $\mathfrak{h}$ symmetry becomes equivalent to the one-dimensional Hubbard model [17]. This conclusion was drawn first by comparison of the Bethe equations of both models and second by comparison of their $R$-matrices. Here we show explicitly how the Hubbard model can be embedded into the $\mathfrak{h}$ spin chain on the level of 
Hamiltonians as a preparation for the more complicated comparison with the Alcaraz-Bariev models.

The problem in the comparison of Hamiltonians consists of the fact that the original Hubbard model Hamiltonian exhibits manifest $\mathfrak{s u ( 2 )}$ symmetry while our Hamiltonian has manifest $\mathrm{U}_{q}(\mathfrak{s u}(2) \times \mathfrak{s u}(2))$ symmetry. We therefore need to recover an additional manifest $\mathfrak{s u}(2)$ symmetry and set $q=1$ before the Hamiltonians can be compared.

Hubbard Hamiltonian. The standard Hubbard model Hamiltonian based on the electronic oscillator algebra (5.33) reads

$$
\mathcal{H}_{j, k}^{\mathrm{Hub}}=\sum_{\alpha=1,2}\left(c_{\alpha, j}^{\dagger} c_{\alpha, k}+c_{\alpha, k}^{\dagger} c_{\alpha, j}\right)+U n_{1, j} n_{2, j} .
$$

It has one manifest $\mathfrak{s u}(2)$ symmetry and a twisted $\mathfrak{s u}(2)$ symmetry. In order to match with our Hamiltonian in table 3, 4 we should first make the second $\mathfrak{s u ( 2 )}$ symmetry manifest by applying a transformation (5.12)

$\mathcal{H}_{j, k}^{\prime}=\mathcal{T}_{j, k} \mathcal{H}_{j, k}^{\mathrm{Hub}} \mathcal{T}_{j, k}^{-1}+\frac{1}{4} U\left(1-n_{1, j}-n_{1, k}-n_{2, j}-n_{2, k}-2 n_{1, j} n_{2, j}+2 n_{1, k} n_{2, k}\right)$

with the twist

$$
\mathcal{T}_{j, k}=\exp \left(\frac{\mathrm{i}}{2} \pi\left(n_{1, k}+n_{2, k}-1\right)\right)=\exp \left(\frac{\mathrm{i}}{2} \pi \mathfrak{H}_{1, k}\right) .
$$

The resulting Hamiltonian reads

$\mathcal{H}_{j, k}^{\prime}=\sum_{\alpha=1,2}\left(-\mathrm{i} c_{\alpha, j}^{\dagger} c_{\alpha, k}+\mathrm{i} c_{\alpha, k}^{\dagger} c_{\alpha, j}\right)+\frac{1}{4} U \sum_{\ell=k, j}\left(n_{1, \ell} n_{2, \ell}+\left(1-n_{1, \ell}\right)\left(1-n_{2, \ell}\right)-\frac{1}{2}\right)$.

It has manifest $\mathrm{U}_{q}(\mathfrak{s u}(2) \times \mathfrak{s u}(2))$ invariance with $q=1$ and takes the form in table 3 with

$$
\begin{aligned}
& A^{\prime}=B^{\prime}=-D^{\prime}=-E^{\prime}=\frac{1}{4} U, \quad G^{\prime}=-L^{\prime}=i, \\
& C^{\prime}, F^{\prime}=-2(\mathrm{i} \kappa)^{ \pm 1}, \quad H^{\prime}=K^{\prime}=0 .
\end{aligned}
$$

Comparison of Hamiltonians. It was shown in [17] that the Hubbard model corresponds to the values $(0, \infty)$ or $(\infty, 0)$ of the parameters $\left(x^{+}, x^{-}\right)$. Moreover we have to set $q=1$ for the undeformed setup.

In fact the Hamiltonian is singular at these parameter values and there are several ways in which the singular limit can be taken. For instance, we can first set $q=1$ and then take the limit

$$
\left(x^{+}, x^{-}\right) \rightarrow(0, \infty) .
$$

Alternatively one can take the limits

$$
\left(x^{+}, x^{-}\right) \rightarrow(0, s(0)) \quad \text { or } \quad\left(x^{+}, x^{-}\right) \rightarrow(s(\infty), \infty)
$$

and only afterwards $q \rightarrow 1$. We rescale the Hamiltonian to match the above $G^{\prime}$

$$
\mathcal{H}_{j, k}^{\prime}=\frac{\mathrm{i}}{G} \mathcal{H}_{j, k}
$$

This leads to, see (5.9),

$$
\begin{aligned}
& A^{\prime}=B^{\prime}=-D^{\prime}=-E^{\prime}=\frac{1}{4 g}, \quad G^{\prime}=-L^{\prime}=i, \\
& C^{\prime}=F^{\prime}=-2, \quad H^{\prime}=K^{\prime}=0,
\end{aligned}
$$

which agrees exactly with the twisted Hubbard Hamiltonian (5.38) upon identification of $\kappa=-\mathrm{i}$ and

$$
U=\frac{1}{g}
$$




\subsection{Connection with the Alcaraz-Bariev models}

Here we study the relation between our model and an integrable spin chain proposed by Alcaraz and Bariev [34] (AB model).

The Alcaraz-Bariev Hamiltonian. Alcaraz and Bariev [34] proposed a spin-chain model based on the electronic states introduced in section 5.3. The Hamiltonian is the following general deformation of the Hubbard Hamiltonian (5.34):

$$
\begin{aligned}
\mathcal{H}_{j, k}^{\mathrm{AB}}=\left(c_{1, j}^{\dagger} c_{1, k}\right. & \left.+c_{1, k}^{\dagger} c_{1, j}\right)\left(1+t_{11} n_{2, j}+t_{12} n_{2, k}+t_{1}^{\prime} n_{2, j} n_{2, k}\right) \\
& +\left(c_{2, j}^{\dagger} c_{2, k}+c_{2, k}^{\dagger} c_{2, j}\right)\left(1+t_{21} n_{1, j}+t_{22} n_{1, k}+t_{2}^{\prime} n_{1, j} n_{1, k}\right) \\
& +J\left(c_{1, j}^{\dagger} c_{2, k}^{\dagger} c_{2, j} c_{1, k}+c_{1, k}^{\dagger} c_{2, j}^{\dagger} c_{2, k} c_{1, j}\right)+t_{\mathrm{p}}\left(c_{1, j}^{\dagger} c_{2, j}^{\dagger} c_{2, k} c_{1, k}+c_{1, j}^{\dagger} c_{2, j}^{\dagger} c_{2, k} c_{1, k}\right) \\
& +V_{11} n_{1, j} n_{1, k}+V_{12} n_{1, j} n_{2, k}+V_{21} n_{2, j} n_{1, k}+V_{22} n_{2, j} n_{2, k}+U n_{1, j} n_{2, j} \\
& +V_{3}^{(1)} n_{2, j} n_{1, k} n_{2, k}+V_{3}^{(2)} n_{1, j} n_{1, k} n_{2, k}+V_{3}^{(3)} n_{1, j} n_{2, j} n_{2, k}+V_{3}^{(4)} n_{1, j} n_{2, j} n_{1, k} \\
& +V_{4} n_{1, j} n_{2, j} n_{1, k} n_{2, k},
\end{aligned}
$$

where

$$
\begin{array}{lll}
t_{11}=t_{4}-1, & t_{12}=t_{3}-1, & t_{1}^{\prime}=t_{5}-t_{3}-t_{4}+1, \\
t_{21}=t_{1}-1, & t_{22}=t_{2}-1, & t_{2}^{\prime}=t_{5}-t_{1}-t_{2}+1 .
\end{array}
$$

It was found to be integrable in four cases which we will denote by $\mathrm{A}^{ \pm}$and $\mathrm{B}^{ \pm}$. The coefficients are related as follows in the case $A^{ \pm}$:

$$
\begin{aligned}
& t_{1}=\epsilon t_{2}=t_{3}=\epsilon t_{4}=\sin \vartheta, \quad t_{5}=\epsilon= \pm 1, \\
& J=-\epsilon t_{\mathrm{p}}=-\frac{1}{2} \epsilon U=V_{12} \mathrm{e}^{2 \eta}=V_{21} \mathrm{e}^{-2 \eta}=\cos \vartheta \\
& V_{11}=V_{22}=V_{3}^{(1)}=V_{3}^{(2)}=V_{3}^{(3)}=V_{3}^{(4)}=V_{4}=0
\end{aligned}
$$

and in the case $\mathrm{B}^{ \pm}$

$t_{1}=\epsilon t_{2}=\epsilon t_{3} \mathrm{e}^{2 \eta}=t_{4} \mathrm{e}^{-2 \eta}=\sin \vartheta, \quad t_{5}=\epsilon= \pm 1$,

$J=-\epsilon t_{\mathrm{p}}=V_{12} \mathrm{e}^{2 \eta}=V_{21} \mathrm{e}^{-2 \eta}=\cos \vartheta, \quad U=2 t_{p}+\sin \vartheta \tan \vartheta\left(e^{\eta}-\epsilon \mathrm{e}^{-\eta}\right)^{2}$,

$V_{11}=V_{22}=V_{3}^{(2)}=V_{3}^{(4)}=V_{4}=0, \quad V_{3}^{(1)}=-V_{3}^{(3)}=V_{12}-V_{21}$

with the free parameters $\vartheta, \eta$. The parameter $\epsilon= \pm 1$ distinguishes between the models $\mathrm{A}^{ \pm}$, $\mathrm{B}^{ \pm}$, respectively.

Let us introduce a replacement of the parameter $\eta$ that will become very useful in the following discussion. In the $\mathrm{A}^{ \pm}$case we shall set

$$
\mathrm{e}^{2 \eta}=\frac{\epsilon}{\xi} \frac{1-\xi \cos \vartheta}{\xi-\cos \vartheta}
$$

while for the case $\mathrm{B}^{ \pm}$we use the definition

$$
\mathrm{e}^{2 \eta}=\epsilon \xi \frac{1-\xi \cos \vartheta}{\xi-1 \cos \vartheta}
$$

$\mathrm{U}_{q}(\mathfrak{s u}(2) \times \mathfrak{s u}(2))$ Symmetry. First of all we note that the AB Hamiltonian does not immediately match the expression of the general $\mathrm{U}_{q}(\mathfrak{s u}(2) \times \mathfrak{s u}(2))$-invariant Hamiltonian in table 3. However, we can apply the transformation (5.12) which preserves integrability in order to restore the $\mathrm{U}_{q}(\mathfrak{s u}(2) \times \mathfrak{s u}(2))$ invariance. 
We find that the $\mathrm{AB}$ Hamiltonian can be brought into the form in table 3 if some of the parameters are related: explicitly we find that the $V_{k}$ parameters must be related by

$$
\begin{array}{rlrl}
V_{11} & =V . & V_{3}^{(1)} & =-\mathrm{e}^{2 \mathrm{i} f_{3}} q J+\mathrm{e}^{2 \mathrm{i} f_{1}} q^{-1} t_{\mathrm{p}}-2 V, \\
V_{12} & =\mathrm{e}^{2 \mathrm{i} f_{3}} q^{-1} J+V . & V_{3}^{(2)} & =-\mathrm{e}^{2 \mathrm{i} f_{3}} q^{-1} J+\mathrm{e}^{2 \mathrm{i} f_{1}} q^{-1} t_{\mathrm{p}}-2 V, \\
V_{21} & =\mathrm{e}^{2 \mathrm{i} f_{3}} q J+V . & V_{3}^{(3)} & =-\mathrm{e}^{2 \mathrm{i} f_{3}} q^{-1} J+\mathrm{e}^{2 \mathrm{i} f_{1}} q t_{\mathrm{p}}-2 V, \\
V_{22} & =V & V & V_{3}^{(4)}=-\mathrm{e}^{2 \mathrm{i} f_{3}} q J+\mathrm{e}^{2 \mathrm{i} f_{1}} q t_{\mathrm{p}}-2 V, \\
V_{4} & =\mathrm{e}^{2 \mathrm{i} f_{3}}\left(q+q^{-1}\right) J-\mathrm{e}^{2 \mathrm{i} f_{1}}\left(q+q^{-1}\right) t_{\mathrm{p}}+4 V,
\end{array}
$$

with a new parameter $V$. Furthermore the $t_{k}$ parameters must take the form

$$
\begin{aligned}
& t_{1}=t_{0}, \quad t_{2}=-\mathrm{e}^{2 \mathrm{i} f_{1}+2 i f_{3}} t_{0}, \quad t_{3}=-\mathrm{e}^{2 \mathrm{i} f_{1}} q^{-1} t_{0}, \\
& t_{4}=\mathrm{e}^{2 \mathrm{i} f_{3}} q t_{0}, \quad t_{5}=-\mathrm{e}^{2 \mathrm{i} f_{1}+2 i f_{3}},
\end{aligned}
$$

with a new parameter $t_{0}$. The parameters for the transformation take the form

$$
\begin{aligned}
& \frac{1}{2} a_{1}=a_{2}=-\frac{1}{4} \mathrm{e}^{2 \mathrm{i} f_{1}}\left(q+q^{-1}\right)-\frac{1}{4} U, \\
& \frac{1}{2} b_{1}=b_{2}=-\frac{1}{4} \mathrm{e}^{2 \mathrm{i} f_{1}}\left(q-q^{-1}\right)-\frac{1}{4} U, \\
& a_{3}=b_{3}=0,
\end{aligned}
$$

and the twist parameters are constrained by

$$
\mathrm{e}^{4 i f_{1}}=\mathrm{e}^{4 i f_{2}}=\mathrm{e}^{4 i f_{3}}=\mathrm{e}^{2 \mathrm{i} f_{3}+2 i f_{2}}=1 .
$$

Note that we have set $a_{0}=1$. We have also adjusted $a_{2}, b_{2}$ such that $H^{\prime}=K^{\prime}=0$. The resulting values for the parameters of table 3 read

$$
\begin{array}{ll}
A^{\prime}=\frac{1}{4} \mathrm{e}^{2 \mathrm{i} f_{1}}\left(q+q^{-1}\right) t_{\mathrm{p}}+\frac{1}{4} U, & D^{\prime}=-\frac{1}{4} \mathrm{e}^{2 \mathrm{i} f_{1}}\left(q+q^{-1}\right) t_{\mathrm{p}}-\frac{1}{4} U+V, \\
B^{\prime}=-\frac{3}{4} \mathrm{e}^{2 \mathrm{i} f_{1}}\left(q+q^{-1}\right) t_{\mathrm{p}}+\frac{1}{4} U, & E^{\prime}=\mathrm{e}^{2 \mathrm{i} f_{3}}\left(q+q^{-1}\right) J+\frac{3}{4} \mathrm{e}^{2 \mathrm{i} f_{1}}\left(q+q^{-1}\right) t_{\mathrm{p}}-\frac{1}{4} U+V, \\
C^{\prime}=-\mathrm{e}^{\mathrm{i} f_{1}+i f_{3}} \kappa t_{0}\left(q+q^{-1}\right), & F^{\prime}=-\mathrm{e}^{-\mathrm{i} f_{1}-i f_{3}} \kappa^{-1} t_{0}\left(q+q^{-1}\right), \\
G^{\prime}=\mathrm{e}^{-\mathrm{i} f_{1}+i f_{2}-i f_{3}}, & L^{\prime}=\mathrm{e}^{+i f_{1}+i f_{2}-i f_{3}}, \\
H^{\prime}=0, & K^{\prime}=0 .
\end{array}
$$

The ten parameters $A^{\prime}, \ldots, L^{\prime}$ are thus given as functions of six parameters $J, U, t_{0}, t_{\mathrm{p}}, V$ and $\kappa$. Three further parameters are implicitly given by $a_{0}, a_{2}$ (overall scaling and identical shift) and $b_{2}$ (affecting $H$ and $K$ only) which preserve the form in table 3 and which we have fixed above. One of the ten parameters cannot be chosen continuously due to the relation $G^{\prime} / L^{\prime}=\mathrm{e}^{2 \mathrm{i} f_{1}}= \pm 1$.

Comparison of Hamiltonians. We find that in the cases $\mathrm{A}^{ \pm}$the symmetry can be restored only if $\mathrm{e}^{2 \eta}=\epsilon$ or $\cos \vartheta=0$. Moreover, $q= \pm 1$. This means that we cannot explain the two-parametric model $\mathrm{A}^{ \pm}$in general using our methods. We shall therefore disregard this case here. In order to relate the cases $\mathrm{A}^{ \pm}$to our model we would have to find a more general transformation than (5.12) to recover the symmetry. It would be interesting to find out if this is possible.

Conversely, the models $\mathrm{B}^{ \pm}$can be brought to the form in table 3 , and in what follows we shall exclusively consider these cases. We match the $t_{k}$ in (5.51), (5.47) by demanding

$$
\epsilon=-\mathrm{e}^{2 \mathrm{i} f_{1}+2 i f_{3}}, \quad q=\mathrm{e}^{2 \eta} \mathrm{e}^{2 \mathrm{i} f_{3}} .
$$


The correct expressions for $V_{k}$ in (5.50) follow by imposing the remaining four relations in (5.47)

$V=0, \quad J=-\epsilon t_{\mathrm{p}}, \quad t_{\mathrm{p}}^{2}+t_{0}^{2}=1, \quad U=2 t_{\mathrm{p}}+\left(t_{\mathrm{p}}-t_{\mathrm{p}}^{-1}\right)\left(\epsilon\left(\mathrm{e}^{2 \eta}+\mathrm{e}^{-2 \eta}\right)-2\right)$.

Incidentally, these are precisely the constraints for our integrable Hamiltonian (5.8) when using the general AB coefficients (5.54). Therefore it should be possible to find suitable parameters $x^{ \pm}, g$ in table 4 to match the case $\mathrm{B}^{ \pm}$.

We start the comparison by making the convenient choice

$f_{1}=-\frac{1}{4} \pi(1+\epsilon), \quad f_{2}=f_{3}=0, \quad \epsilon=-\mathrm{e}^{2 \mathrm{i} f_{1}}, \quad q=\mathrm{e}^{2 \eta}$.

To match $G^{\prime}=\mathrm{e}^{-\mathrm{i} f_{1}}$ we have rescaled our Hamiltonian in tables 3 and 4:

$$
\mathcal{H}_{j, k}^{\prime}=\frac{\mathrm{e}^{-\mathrm{i} f_{1}}}{G} \mathcal{H}_{j, k}
$$

Furthermore we have to match our normalization $C^{\prime}=F^{\prime}$ by setting

$$
\kappa=\mathrm{e}^{-\mathrm{i} f_{1}} .
$$

The ratio $L^{\prime} / G^{\prime}=L / G=\mathrm{e}^{2 \mathrm{i} f_{1}}=-\epsilon$ is determined through the $x^{ \pm}$,

$$
\frac{L}{G}=q^{-1} \frac{x^{+}-s\left(x^{+}\right)}{x^{-}-s\left(x^{-}\right)}=-\epsilon .
$$

This constraint is satisfied when (our) $U^{2}=-\epsilon$ or when $q^{2 C}=-\epsilon$. Both values are possible, and let us discuss them separately:

The condition $q^{2 C}=-\epsilon$ holds for the following two pairs $\left(x^{+}, x^{-}\right)$:

$$
\begin{aligned}
& x^{+}=\frac{\mathrm{i} q}{2 g} \frac{1 \pm \sqrt{1-4 \epsilon g^{2}\left(q^{1 / 2}-\epsilon q^{-1 / 2}\right)^{2}}}{1-\epsilon q}, \\
& x^{-}=\frac{\epsilon \mathrm{i}}{2 g} \frac{1 \mp \sqrt{1-4 \epsilon g^{2}\left(q^{1 / 2}-\epsilon q^{-1 / 2}\right)^{2}}}{1-\epsilon q} .
\end{aligned}
$$

We match $\vartheta$ of $\mathrm{B}^{ \pm}$using the coefficients $A^{\prime}-B^{\prime}$ or $C^{\prime}$

$$
\begin{aligned}
& \cos \vartheta=-\epsilon t_{\mathrm{p}}=\frac{B^{\prime}-A^{\prime}}{\mathrm{e}^{2 \eta}+\mathrm{e}^{-2 \eta}}=\frac{\mathrm{e}^{-\mathrm{i} f_{1}}}{\mathrm{e}^{2 \eta}+\mathrm{e}^{-2 \eta}} \frac{B-A}{G}= \pm \frac{g\left(\mathrm{e}^{\eta}-\epsilon \mathrm{e}^{-\eta}\right)^{2}}{\sqrt{1-4 \epsilon g^{2}\left(\mathrm{e}^{\eta}-\epsilon \mathrm{e}^{-\eta}\right)^{2}}}, \\
& \sin \vartheta=t_{0}=-\frac{C^{\prime}}{\mathrm{e}^{2 \eta}+\mathrm{e}^{-2 \eta}}=-\frac{\mathrm{e}^{-\mathrm{i} f_{1}}}{\mathrm{e}^{2 \eta}+\mathrm{e}^{-2 \eta}} \frac{C}{G}=\mp \frac{\sqrt{1-g^{2}\left(\mathrm{e}^{2 \eta}-\mathrm{e}^{-2 \eta}\right)^{2}}}{\sqrt{1-4 \epsilon g^{2}\left(\mathrm{e}^{\eta}-\epsilon \mathrm{e}^{-\eta}\right)^{2}}} .
\end{aligned}
$$

These two relations are compatible with the identity $\cos ^{2} \vartheta+\sin ^{2} \vartheta=1$ and they can be solved for $g$,

$$
g=\frac{\cos \vartheta}{4 \cosh \left(\eta+\mathrm{i} f_{1}\right) \sqrt{\cosh ^{2}\left(\eta+\mathrm{i} f_{1}\right)-\cos ^{2} \vartheta}} .
$$

In the parametrization (5.49) the above relations simplify significantly

$$
g=\frac{\xi(1-\xi \cos \vartheta)(\xi-\cos \vartheta)}{\cos \vartheta\left(\xi-\mathrm{e}^{+\mathrm{i} \vartheta}\right)\left(\xi-\mathrm{e}^{-\mathrm{i} \vartheta}\right)\left(\xi^{2}-1\right)}, \quad q=\epsilon \xi \frac{1-\xi \cos \vartheta}{\xi-\cos \vartheta}, \quad x^{ \pm}=\mathrm{i} \epsilon \xi^{ \pm 1} .
$$


Conversely, the condition $U^{2}=-\epsilon$ holds for the following two pairs $\left(x^{+}, x^{-}\right)$(note the change of sign with respect to (5.61)):

$$
\begin{aligned}
& x^{+}=\frac{\mathrm{i} q}{2 g} \frac{1 \pm \sqrt{1+4 \epsilon g^{2}\left(q^{1 / 2}+\epsilon q^{-1 / 2}\right)^{2}}}{1+\epsilon q}, \\
& x^{-}=\frac{-\epsilon i}{2 g} \frac{1 \pm \sqrt{1+4 \epsilon g^{2}\left(q^{1 / 2}+\epsilon q^{-1 / 2}\right)^{2}}}{1+\epsilon q} .
\end{aligned}
$$

We match $\vartheta$ of $\mathrm{B}^{ \pm}$using the coefficients $A^{\prime}-B^{\prime}$ or $C^{\prime}$

$$
\begin{aligned}
& \cos \vartheta=-\epsilon t_{\mathrm{p}}=\frac{B^{\prime}-A^{\prime}}{\mathrm{e}^{2 \eta}+\mathrm{e}^{-2 \eta}}=\frac{\mathrm{e}^{-\mathrm{i} f_{1}}}{\mathrm{e}^{2 \eta}+\mathrm{e}^{-2 \eta}} \frac{B-A}{G}= \pm \frac{\sqrt{1-g^{2}\left(\mathrm{e}^{2 \eta}-\mathrm{e}^{-2 \eta}\right)^{2}}}{\sqrt{1+4 \epsilon g^{2}\left(\mathrm{e}^{\eta}+\epsilon \mathrm{e}^{-\eta}\right)^{2}}} \\
& \sin \vartheta=t_{0}=-\frac{C^{\prime}}{\mathrm{e}^{2 \eta}+\mathrm{e}^{-2 \eta}}=-\frac{\mathrm{e}^{-\mathrm{i} f_{1}}}{\mathrm{e}^{2 \eta}+\mathrm{e}^{-2 \eta}} \frac{C}{G}= \pm \frac{g\left(\mathrm{e}^{\eta}+\epsilon \mathrm{e}^{-\eta}\right)^{2}}{\sqrt{1+4 \epsilon g^{2}\left(\mathrm{e}^{\eta}+\epsilon \mathrm{e}^{-\eta}\right)^{2}}} .
\end{aligned}
$$

These two relations are compatible with the identity $\cos ^{2} \vartheta+\sin ^{2} \vartheta=1$ and they can be solved for $g$ :

$$
g=\frac{\sin \vartheta}{4 \sinh \left(\eta+\mathrm{i} f_{1}\right) \sqrt{\sinh ^{2}\left(\eta+\mathrm{i} f_{1}\right)+\sin ^{2} \vartheta}} .
$$

Again the parametrization (5.49) simplifies the above relations

$$
\begin{aligned}
g & =\frac{\sin \vartheta(1-\xi \cos \vartheta)(\xi-\cos \vartheta)}{\cos \vartheta\left(\xi-\mathrm{e}^{+\mathrm{i} \vartheta}\right)\left(\xi-\mathrm{e}^{-\mathrm{i} \vartheta}\right)\left(\left(\xi+\xi^{-1}\right) \cos \vartheta-2\right)}, \\
q & =\epsilon \xi \frac{1-\xi \cos \vartheta}{\xi-\cos \vartheta}, \quad x^{+}=\frac{\mathrm{i} \epsilon \xi \sin \vartheta}{\cos \vartheta-\xi}, \quad x^{-}=\frac{\mathrm{i} \epsilon \sin \vartheta}{1-\xi \cos \vartheta}
\end{aligned}
$$

Note that several different points in the constrained parameter space of $x^{ \pm}, g$ correspond to the same Hamiltonian given in terms of $\vartheta$. Furthermore, we have not been very careful about selection of branches of $U=\sqrt{U^{2}}, q^{C}=\sqrt{q^{2 C}}$ in (2.62)-(2.64) as well as for the above square roots. We expect that these signs ambiguities are equivalent to shifting $\vartheta$ and i $\eta$ by multiples of $\pi / 2$.

Comparison of Bethe equations. Here we will show how the $\mathrm{B}^{ \pm}$models proposed by Alcaraz and Bariev [34] can be embedded into our $\mathrm{U}_{q}(\mathfrak{h})$-spin-chain model by comparing Bethe equations.

Consider the Alcaraz-Bariev Bethe equations [34]

$$
\begin{aligned}
& 1=\left(z_{k}\right)^{K} \prod_{j=1}^{M} \frac{\sinh \left(\lambda_{k}-\lambda_{j}^{(1)}+\eta\right)}{\sinh \left(\lambda_{k}-\lambda_{j}^{(1)}-\eta\right)}, \\
& 1=\prod_{j=1}^{N} \frac{\sinh \left(\lambda_{k}^{(1)}-\lambda_{j}+\eta\right)}{\sinh \left(\lambda_{k}^{(1)}-\lambda_{j}-\eta\right)} \prod_{\substack{j=1 \\
j \neq k}}^{M} \frac{\sinh \left(\lambda_{k}^{(1)}-\lambda_{j}^{(1)}-2 \eta\right)}{\sinh \left(\lambda_{k}^{(1)}-\lambda_{j}^{(1)}+2 \eta\right)} .
\end{aligned}
$$

By inserting $\Phi(z)$ into the expression for $\exp \left(2 \lambda_{k}\right)$ in [34] we can write the relation between $z_{k}$ and $\lambda_{k}$ as follows:

$$
\exp \left(2 \lambda_{k}\right)=\mathrm{e}^{-2 \eta} \frac{z_{k}^{2}+\left(\epsilon \mathrm{e}^{+2 \eta}-1\right) t_{\mathrm{p}}^{-1} z_{k}-\epsilon \mathrm{e}^{+2 \eta}}{z_{k}^{2}+\left(\epsilon \mathrm{e}^{-2 \eta}-1\right) t_{\mathrm{p}}^{-1} z_{k}-\epsilon \mathrm{e}^{-2 \eta}} .
$$

The energy of an eigenstate is given by

$$
E=\sum_{j=1}^{N}\left(z_{j}+z_{j}^{-1}\right) \text {. }
$$


It is straightforward to match the auxiliary (second) Bethe equation with our Bethe equations (5.16) where $f_{2}=f_{3}=0$ by equating $q=\mathrm{e}^{2 \eta}$ and

$$
\begin{aligned}
& u\left(y_{k}\right)=\exp \left(2 \lambda_{k}-2 \lambda_{0}\right)-\frac{\mathrm{i} g^{-1}}{q-q^{-1}}, \\
& w_{k}=\exp \left(2 \lambda_{k}^{(1)}-2 \lambda_{0}\right)-\frac{\mathrm{i}}{2} g^{-1} \frac{q+q^{-1}}{q-q^{-1}} .
\end{aligned}
$$

Matching of the first Bethe equation requires

$$
z_{k}=\mathrm{e}^{-\mathrm{i} f_{1}} q^{-C-1 / 2} U^{-1} \frac{y_{k}-x^{+}}{y_{k}-x^{-}}, \quad y_{k}=\frac{x^{+}-\mathrm{e}^{\mathrm{i} f_{1}} q^{C+1 / 2} U x^{-} z_{k}}{1-\mathrm{e}^{\mathrm{i} f_{1}} q^{C+1 / 2} U z_{k}} .
$$

We can bring the above relations (5.72), (5.73) between $\lambda_{k}$ and $y_{k}$ in the same form as (5.70)

$\exp \left(2 \lambda_{k}\right)=\exp \left(2 \lambda_{0}\right)\left(u\left(y_{k}\right)+\frac{\mathrm{i} g^{-1}}{q-q^{-1}}\right)=\exp \left(2 \lambda_{0}\right) \frac{\mathrm{i} g^{-1}}{q-q^{-1}} y_{k} s\left(y_{k}\right)$

with

$y_{k} s\left(y_{k}\right)=q^{-2} x^{+} s\left(x^{+}\right) \frac{z_{k}^{2}-\mathrm{e}^{-\mathrm{i} f_{1}} U^{-1} q^{C+1 / 2}\left(2+\left(U^{2}-q^{-2 C}\right) / q^{-1} x^{+} s\left(x^{+}\right)\right) z_{k}+\mathrm{e}^{-2 \mathrm{i} f_{1}} q}{z_{k}^{2}-\mathrm{e}^{-\mathrm{i} f_{1}} U^{-1} q^{C-1 / 2}\left(U^{2}+q^{-2 C}\right) z_{k}+\mathrm{e}^{-2 \mathrm{i} f_{1}} q^{-1}}$.

We set $q^{2 C}=\mathrm{e}^{2 \mathrm{i} f_{1}}=-\epsilon$ and use (5.61). Noting that $q^{-1} x^{+} s\left(x^{+}\right)=q x^{-} s\left(x^{-}\right)=-\epsilon$ the prefactors imply that we have to choose the shift parameter $\lambda_{0}$ as follows:

$$
\exp \left(2 \lambda_{0}\right)=\epsilon \operatorname{ig}\left(q-q^{-1}\right) \text {. }
$$

Some further manipulations then show that our relation $(5.74),(5.75)$ is precisely the same as the one from the Alcaraz-Bariev equations (5.70). Finally, the eigenstate energies $E$ (5.71) match with our result $E^{\prime}$ (5.17), (5.19), (5.23) using the transformation parameters

$$
a_{0}=\mathrm{e}^{\mathrm{i} f_{1}} \frac{1}{G}, \quad a_{1}=2 \mathrm{e}^{\mathrm{i} f_{1}} \frac{A}{G}, \quad a_{2}=\mathrm{e}^{\mathrm{i} f_{1}} \frac{A}{G}, \quad a_{3}=0 .
$$

The spectrum of the $A^{ \pm}$cases. The Bethe equations derived by Alcaraz and Bariev are almost the same for the $\mathrm{A}^{ \pm}$and $\mathrm{B}^{ \pm}$cases. Actually, the only difference is the definition of a global parameter $r$ as a function of $\eta$, namely

$\cosh 2 r=\epsilon \sin ^{2} \vartheta+\cos ^{2} \vartheta \cosh 2 \eta \quad$ versus $\quad \cosh 2 r=\cosh 2 \eta$

for cases A and B, respectively. This implies that set of solutions to the Bethe equations for model A with $\eta=\eta_{\mathrm{A}}$ is the same as the set of solutions for model $\mathrm{B}$ with $\eta=\eta_{\mathrm{B}}$ where $\eta_{\mathrm{A}}$ and $\eta_{\mathrm{B}}$ are related by

$$
\epsilon \sin ^{2} \vartheta+\cos ^{2} \vartheta \cosh 2 \eta_{\mathrm{A}}=\cosh 2 \eta_{\mathrm{B}}
$$

Moreover the dispersion relation is the same for both cases, and therefore the spectra of the models agree. Note that our reparametrization (5.48), (5.49) of $\eta$ has been chosen carefully so that the above relation holds for equal $\xi=\xi_{\mathrm{A}}=\xi_{\mathrm{B}}$.

In fact this leads to a puzzle concerning our above results: we have not been able to find a transformation of the Hamiltonian for model A that restores manifest $\mathrm{U}_{q}(\mathfrak{s u}(2) \times \mathfrak{s u}(2))$ symmetry. Therefore we have not been able to relate this Hamiltonian to ours. Nevertheless the matching of spectra implies that the two Hamiltonians are indeed related by a similarity transformation for a suitable choice of parameters. Consequently, there must exist a more 
elaborate transformation to make the $\mathrm{U}_{q}(\mathfrak{s u}(2) \times \mathfrak{s u}(2))$ symmetry of the Hamiltonian of model A manifest and match it with our Hamiltonian.

Comparison of scattering matrices. To shed more light onto the equivalence of the $\mathrm{A}^{ \pm}$and $\mathrm{B}^{ \pm}$ cases we shall compare the corresponding scattering matrices. These were derived in [34] for excitations $c_{1}^{\dagger}$ and $c_{2}^{\dagger}$ with momentum $p=-\mathrm{i} \log z$ above the (ferromagnetic) vacuum and they can be compared to the results of section 4.2. We will use the parametrization (5.48), (5.49) which allows us to compare the expressions directly without the further need to transform parameters. It turns out that the diagonal scattering matrix elements precisely match

$$
\left(S_{\mathrm{A}}\right)_{12}^{12}\left(z_{1}, z_{2}\right)=\left(S_{\mathrm{A}}\right)_{21}^{21}\left(z_{1}, z_{2}\right)=\left(S_{\mathrm{B}}\right)_{12}^{12}\left(z_{1}, z_{2}\right)=\left(S_{\mathrm{B}}\right)_{21}^{21}\left(z_{1}, z_{2}\right)
$$

The off-diagonal elements also agree up to a simple factor

$\left(S_{\mathrm{A}}\right)_{21}^{12}\left(z_{1}, z_{2}\right)=\left(S_{\mathrm{B}}\right)_{21}^{12}\left(z_{1}, z_{2}\right) \frac{f\left(z_{1}\right)}{f\left(z_{2}\right)}, \quad\left(S_{\mathrm{A}}\right)_{12}^{21}\left(z_{1}, z_{2}\right)=\left(S_{\mathrm{B}}\right)_{12}^{21}\left(z_{1}, z_{2}\right) \frac{f\left(z_{2}\right)}{f\left(z_{1}\right)}$

with

$$
f(z)=\frac{1+\epsilon \xi z}{z+\epsilon \xi}
$$

The fact that the off-diagonal elements differ by reciprocal factors of $f\left(z_{1}\right) / f\left(z_{2}\right)$ alone implies that the spectra are the same. Therefore there must exist a similarity transformation between the two Hamiltonians. In fact the transformation is simple for scattering states: each excitation $c_{2}^{\dagger}$ with momentum $p$ is multiplied by a factor of $f\left(\mathrm{e}^{\mathrm{i} p}\right)$ while the other excitations $c_{1}^{\dagger}$ are left alone. It is not hard to convince oneself that this leads to the above relative factors between the $S$-matrix elements. However, it is not as straightforward to express the similarity transformation for spin-chain states because the transformation must be non-local due to the momentum dependence of the factors $f\left(\mathrm{e}^{\mathrm{i} p}\right)$. Astonishingly the Hamiltonian remains local after the similarity transformation which implies that the transformation must be of a very special kind.

An alternative coalgebra? The above discussion has made it clear that the $\mathrm{A}^{ \pm}$and $\mathrm{B}^{ \pm}$ Hamiltonians are equivalent. Consequently the $\mathrm{A}^{ \pm}$Hamiltonian must obey a $\mathrm{U}_{q}(\mathfrak{s u}(2) \times \mathfrak{s u}(2))$ symmetry albeit in a non-standard form. It is conceivable that this symmetry is generated by an alternative coproduct $\mathrm{U}_{q}(\mathfrak{h})$. This is in fact a promising idea because it would explain the locality of the $\mathrm{A}^{ \pm}$Hamiltonian: assuming that there exists an alternative (fundamental) $R$-matrix, we could right away derive a nearest-neighbor Hamiltonian which is likely to be the one of the $\mathrm{A}^{ \pm}$cases. Thus it would be exciting to find an alternative coalgebra structure for $\mathrm{U}_{q}(\mathfrak{h})$ and also find a suitable similarity transformation to the canonical one derived in this paper. Unfortunately this issue is outside the scope of the present paper.

\subsection{Quantum-deformed EKS model}

At the $\left(x^{+}, x^{-}\right)$values $(0,0),(\infty, \infty)$ as well as $(s(0), s(0)),(s(\infty), s(\infty))$ one obtains the quantum deformation of the EKS model [25]. For instance at $\left(x^{+}, x^{-}\right)=(0,0)$ the parameters of the rescaled Hamiltonian $\mathcal{H}_{j, k}^{\prime}=\left(q^{1 / 2} / G\right) \mathcal{H}_{j, k}$ read

$$
\begin{aligned}
& A^{\prime}=-B^{\prime}=-D^{\prime}=E^{\prime}=\frac{1}{2}\left(q+q^{-1}\right), \\
& G=L^{-1}=q^{1 / 2}, \quad C^{\prime}=F^{\prime}=H^{\prime}=K^{\prime}=0 .
\end{aligned}
$$

It is not too hard to see that after a twist this leads to the quantum-deformed EKS model, i.e. a standard integrable nearest-neighbor spin chain with $\mathrm{U}_{q}(\mathfrak{u}(2 \mid 2))$ symmetry and spins in the four-dimensional fundamental representation. 
Note that this model is somewhat singular. For instance Bethe equations (5.16) do not apply directly, but the limit $\left(x^{+}, x^{-}\right) \rightarrow(0,0)$ has to be taken carefully. In particular, there are two allowed limits of the $y_{k}$, either $y_{k} \rightarrow 0$ or $y_{k} \rightarrow s(0)$. Let $N_{1}$ of them be of the first kind and $N_{3}=N-N_{1}$ of the second. They should scale like

$$
y_{k} \sim \exp \left(2 \lambda_{k}^{(1)}\right) q x^{-}, \quad y_{k+N_{1}} \sim s\left(\exp \left(2 \lambda_{k}^{(3)}\right) q x^{-}\right) .
$$

Furthermore, the $w_{k}$ must scale like

$$
w_{k} \sim-\frac{\mathrm{i}}{2 g} \frac{q+q^{-1}}{q-q^{-1}}+\frac{\exp \left(2 \lambda_{k}^{(2)}\right) q x^{-}}{g^{2}\left(q-q^{-1}\right)^{2}} .
$$

The limit of the Bethe equations (5.16) agrees with the standard form of $\mathrm{U}_{q}(\mathfrak{u}(2 \mid 2))$ Bethe equations, cf [46], up to a twist

$$
\begin{aligned}
1 & =\mathrm{e}^{+K \hbar}\left(\frac{\sinh \left(\lambda_{k}^{(1)}-\hbar\right)}{\sinh \left(\lambda_{k}^{(1)}+\hbar\right)}\right)^{K} \prod_{j=1}^{M} \frac{\sinh \left(\lambda_{k}^{(1)}-\lambda_{j}^{(2)}+\hbar\right)}{\sinh \left(\lambda_{k}^{(1)}-\lambda_{j}^{(2)}-\hbar\right)} \\
1 & =\prod_{j=1}^{N_{1}} \frac{\sinh \left(\lambda_{k}^{(2)}-\lambda_{j}^{(1)}+\hbar\right)}{\sinh \left(\lambda_{k}^{(2)}-\lambda_{j}^{(1)}-\hbar\right)} \prod_{\substack{j=1 \\
j \neq k}}^{M} \frac{\sinh \left(\lambda_{k}^{(2)}-\lambda_{j}^{(2)}-2 \hbar\right)}{\sinh \left(\lambda_{k}^{(2)}-\lambda_{j}^{(2)}+2 \hbar\right)} \prod_{j=1}^{N_{3}} \frac{\sinh \left(\lambda_{k}^{(2)}-\lambda_{j}^{(3)}+\hbar\right)}{\sinh \left(\lambda_{k}^{(2)}-\lambda_{j}^{(3)}-\hbar\right)} \\
1 & =\mathrm{e}^{-K \hbar} \prod_{j=1}^{M} \frac{\sinh \left(\lambda_{k}^{(3)}-\lambda_{j}^{(2)}+\hbar\right)}{\sinh \left(\lambda_{k}^{(3)}-\lambda_{j}^{(2)}-\hbar\right)}
\end{aligned}
$$

with $q=\mathrm{e}^{2 \hbar}$. Here the two different kinds of $y_{k}$ have led to the additional Bethe equation needed for standard $\mathrm{U}_{q}(\mathfrak{u}(2 \mid 2))$ chains.

Finally, the limit of the total momentum (5.17) and the total energy (5.19) yields

$$
\begin{aligned}
& \mathrm{e}^{\mathrm{i} P}=\mathrm{e}^{\hbar\left(N_{3}-N_{1}\right)} \prod_{j=1}^{N_{1}} \frac{\sinh \left(\lambda_{k}^{(1)}+\hbar\right)}{\sinh \left(\lambda_{k}^{(1)}-\hbar\right)} \\
& E=\cosh (2 \hbar) K+\sum_{j=1}^{N_{1}} \frac{\sinh ^{2}(2 \hbar)}{\sinh \left(\lambda_{k}^{(1)}+\hbar\right) \sinh \left(\lambda_{k}^{(1)}-\hbar\right)} .
\end{aligned}
$$

We observe that only the $\lambda_{k}^{(1)}$ carry momentum and energy, the $\lambda_{k}^{(3)}$ do not contribute.

\subsection{Another quantum deformation of the Hubbard model}

Some curious values for the parameters $\left(x^{+}, x^{-}\right)$appear to be $(0, s(0)),(s(\infty), \infty)$ and $(s(0), 0)(\infty, s(\infty))$. In the limit $q \rightarrow 1$ they obviously approach the values $(0, \infty)$ and $(\infty, 0)$ corresponding to the Hubbard model. One has to be careful in using these values because several common combinations of $x^{ \pm}$turn out to be singular and have to be regularized properly, e.g. by taking a limit.

For definiteness we shall use the pair

$$
\left(x^{+}, x^{-}\right)=(0, s(0))
$$

the other combinations yield similar results. For convenience we rescale the Hamiltonian

$$
\mathcal{H}_{j, k}^{\prime}=\frac{\mathrm{i} q^{1 / 2}}{G} \mathcal{H}_{j, k}
$$


The regularized coefficients of this Hamiltonian read

$A^{\prime}=B^{\prime}=-D^{\prime}=-E^{\prime}=\frac{1-2\left(q-q^{-1}\right)^{2} g^{2}}{4 g \sqrt{1-\left(q-q^{-1}\right)^{2} g^{2}}}$,

$C^{\prime}=F^{\prime}=q+q^{-1}, \quad G^{\prime}=+\mathrm{i} q^{+1 / 2}, \quad L^{\prime}=-\mathrm{i} q^{-1 / 2}, \quad H^{\prime}=K^{\prime}=0$.

In particular, we find $A^{\prime}=B^{\prime}$ and $D^{\prime}=E^{\prime}$. This is interesting because the pair-hopping terms of the oscillator Hamiltonian in table 5 vanish just as for the Hubbard model (we set $\kappa=-\mathrm{i}$ for convenience)

$$
\begin{aligned}
\mathcal{H}_{j, k}^{\prime}=A^{\prime} \sum_{\ell=j, k} & \left(\left(1-n_{1, \ell}\right)\left(1-n_{2, \ell}\right)+n_{1, \ell} n_{2, \ell}-\frac{1}{2}\right) \\
& +\mathrm{i} q^{+1 / 2} c_{1, j}^{\dagger} c_{1, k}\left(1-\left(1-q^{+1 / 2}\right) n_{2, j}\right)\left(1-\left(1-q^{-3 / 2}\right) n_{2, k}\right) \\
& +\mathrm{i} q^{+1 / 2} c_{2, j}^{\dagger} c_{2, k}\left(1-\left(1-q^{-1 / 2}\right) n_{1, j}\right)\left(1-\left(1-q^{-1 / 2}\right) n_{1, k}\right) \\
& -\mathrm{i} q^{-1 / 2} c_{1, k}^{\dagger} c_{1, j}\left(1-\left(1-q^{+3 / 2}\right) n_{2, j}\right)\left(1-\left(1-q^{-1 / 2}\right) n_{2, k}\right) \\
& -\mathrm{i} q^{-1 / 2} c_{2, k}^{\dagger} c_{2, j}\left(1-\left(1-q^{+1 / 2}\right) n_{1, j}\right)\left(1-\left(1-q^{+1 / 2}\right) n_{1, k}\right)
\end{aligned}
$$

Note that this Hamiltonian is not obviously Hermitian. It would be interesting to find out if its spectrum is nevertheless real (for some non-Hermitian twist).

The closed chain with this Hamiltonian can be diagonalized by the Bethe equations (5.16). The regularized momentum relation $(5.17)$ reads

$$
\mathrm{e}^{\mathrm{i} p_{k}}=\mathrm{i} q^{1 / 2} g \frac{q-q^{-1}+\mathrm{i} g^{-1} / y_{k}}{\sqrt{1-\left(q-q^{-1}\right)^{2} g^{2}}}
$$

and the energy of an eigenstate (5.19) takes the form

$$
E^{\prime}=\frac{1-2\left(q-q^{-1}\right)^{2} g^{2}}{4 g \sqrt{1-\left(q-q^{-1}\right)^{2} g^{2}}}(K-2 N)+\sum_{j=1}^{N}\left(\mathrm{i} q^{+1 / 2} \mathrm{e}^{\mathrm{i} p_{j}}-\mathrm{i} q^{-1 / 2} \mathrm{e}^{-\mathrm{i} p_{j}}\right) .
$$

Note that this dispersion relation is not isotropic. This fact is related to our choice of $x^{ \pm}$. For example, choosing $x^{ \pm}$such that the dispersion relation is isotropic leads to the Alcaraz-Bariev model discussed in section 5.5. It may be desirable to investigate this model further to see if it has interesting (physical) properties.

\section{Conclusions and outlook}

In this paper we have considered quantum deformations of the threefold central extension $\mathfrak{h}=\mathfrak{p s u}(2 \mid 2) \ltimes \mathbb{R}^{3}$ of the Lie superalgebra $\mathfrak{p s u}(2 \mid 2)$. We have set up the Hopf algebra $\mathrm{U}_{q}(\mathfrak{h})$ together with its fundamental $R$-matrix and applied it to derive quantum deformations of the one-dimensional Hubbard model.

A first important result is that the quantum deformation $U_{q}(\mathfrak{h})$ of the Lie superalgebra $\mathfrak{h}$ is possible despite its non-standard structure (central extension). Moreover, we have constructed an invariant fundamental $R$-matrix which obeys the Yang-Baxter equation, crossing symmetry and fusion (with a suitable choice of overall phase factor). This suggests that the Hopf algebra can be made quasi-cocommutative and quasi-triangular. Curiously, the matrix structure of the fundamental $R$-matrix is determined by $\mathrm{U}_{q}(\mathfrak{h})$ invariance alone. This feature is related to the representation theory $\mathrm{U}_{q}(\mathfrak{h})$ which we have outlined and which is largely analogous to the undeformed superalgebras $\mathfrak{h}$ and $\mathfrak{s u}(2 \mid 2)$. We have also discussed Hermiticity conditions and we were forced to choose $q$ to be real. Usually in quantum groups $\mathrm{U}_{q}(\mathfrak{g})$ one can also 
choose $q$ from the unit circle (such that $q+q^{-1}$ is real), and it would be important to understand if and how Hermiticity can be achieved in that case for our algebra.

We have applied the nested Bethe ansatz to the $R$-matrix in order to diagonalize it and to write down the Bethe equations for a closed spin chain. The Bethe equations are structurally similar to the Lieb-Wu equations for the one-dimensional Hubbard model: one should view the former as the quantum deformation of the latter; the Lieb-Wu equations are of the algebraic (XXX-like) type, while ours are trigonometric (XXZ-like). A question for future work is whether an elliptic (XYZ-like) deformation of our fundamental $R$-matrix and the Lieb-Wu equations exists.

In the final section we have derived an integrable Hamiltonian from the $R$-matrix and compared directly it to the one-dimensional Hubbard model and some of its generalizations. Our Hamiltonian has manifest $\mathrm{U}_{q}(\mathfrak{s u}(2) \times \mathfrak{s u}(2))$ symmetry, three independent parameters and it can be further deformed in several canonical ways (rescaling, shifts, twists) while preserving integrability. By adjusting the parameters we were able to match the one-parametric Hubbard chain as well the cases $\mathrm{B}^{ \pm}$of a two-parametric Hamiltonian proposed by Alcaraz and Bariev [34]. We have also identified a potentially interesting combination of parameters for which our Hamiltonian has no pair-hopping terms. We have not managed to explain the cases $\mathrm{A}^{ \pm}$ of the Alcaraz-Bariev Hamiltonian in general, but we have provided an argument based on the Bethe equations why this should be possible nevertheless. It would therefore be important to show explicitly how to relate the cases $\mathrm{A}^{ \pm}$, perhaps by finding an alternative coalgebra for $\mathrm{U}_{q}(\mathfrak{h}(2))$. Furthermore an investigation of the possible condensed matter theory applications of quantum deformations of the one-dimensional Hubbard model is worth performing. Do these models also display signs of superconductivity like the undeformed model? It is also worth finding out if any of the other electronic models discussed in the introduction can be obtained from our Hamiltonian.

Here we have investigated the Hopf algebra $U_{q}(\mathfrak{h})$, but the analysis is far from complete. In fact, the symmetry algebra in the undeformed setup is much larger, and it appears to be a Yangian (double) [21]. The corresponding quantum deformation would be a quantum affine algebra. One will need this algebra in order to formulate the universal $R$-matrix of which the $R$-matrix derived in this paper is the fundamental representation. A classical and undeformed analysis [24] has shown that the affine algebra is not just $\mathfrak{h}\left[u, u^{-1}\right]$. It is rather a deformation of $\mathfrak{u}(2 \mid 2)\left[u, u^{-1}\right]$ which includes only one tower of central charges and one tower of inner automorphisms. Therefore it is quite clear that this quantum affine algebra is not Kac-Moody, and one cannot directly apply the general framework associated with such algebras.

Finally, we should ask how our results can be applied to the AdS/CFT correspondence. Quantum-deformed (XXZ-like) spin chains with $\mathrm{U}_{q}(\mathfrak{s u}(2))$ and $\mathrm{U}_{q}(\mathfrak{s u}(3))$ symmetry have indeed appeared in this context, in particular for so-called beta-deformed $\mathcal{N}=4$ gauge theory [47] with a complex deformation parameter [48-50]. However, the argument used in [49] tells us that the symmetry considered in this paper cannot apply to conformal gauge theories such as [47]: the two bosonic subalgebras $\mathfrak{s u}(2)$ of $\mathfrak{p s u}(2 \mid 2)$ play different roles; one originates from the internal symmetry which is deformed, and the other one originates from conformal symmetry which remains undeformed. In contrast, quantum deformations typically apply to the whole of a Hopf algebra, as is the case for our algebra. Nevertheless, it seems possible to deform the internal $\mathfrak{s u ( 4 )}$ symmetry and the associated $S^{5}$, so why should it not be possible to deform $\mathfrak{s u}(2,2)$ and the associated $\mathrm{AdS}_{5}$ ? Let us speculate about such a complete quantum deformation of the AdS/CFT correspondence: an 'AdS $\mathrm{ACFT}_{q}$ ' duality. The field theory would have to have quantum-deformed conformal and internal symmetries, and it would most probably be formulated on some sort of non-commutative spacetime. The string theory would be formulated on a quantum-deformed $\left(\operatorname{AdS}_{5} \times S^{5}\right)_{q}$ background, which 
would be both curved and non-commutative. Perhaps the most convenient definition would be in terms of coset spaces such as $\mathrm{SO}_{q}(6) / \mathrm{SO}_{q}(5)$ with non-commutative coordinates. Despite the expected dire technical complications, the planar limit of these dual models would stand a good chance of being integrable, and the world sheet $S$-matrix would then be given through our quantum-deformed $R$-matrix. It would be exciting to find out if this proposed picture is more than daydreaming.

\section{Acknowledgments}

The authors are grateful to thank Fabian Spill for collaboration at earlier stages of the project and for many discussions. The authors would also like to thank Sergey Frolov, Frank Göhmann, Alexander Gorsky for fruitful discussions. P K thanks MPI für Gravitationsphysik in Potsdam for hospitality where main part of this work was performed. The work of $\mathrm{P} \mathrm{K}$ was partially supported by a grant of the President of Russian Federation (NS-7293.2006.2) and a grant of the Dynasty Foundation awarded by the Scientific Board of ICFPM.

\section{Appendix A. Some useful relations}

The fundamental representation is defined in terms of the variables $x^{ \pm}$constrained by the quadratic relation $(2.61)$

$$
\frac{x^{+}}{q}+\frac{q}{x^{+}}-q x^{-}-\frac{1}{q x^{-}}+\mathrm{i} g\left(q-q^{-1}\right)\left(\frac{x^{+}}{q x^{-}}-\frac{q x^{-}}{x^{+}}\right)=\frac{\mathrm{i}}{g} .
$$

An expression which depends on $x^{ \pm}$can therefore be written in many ways which are equivalent upon (A.1). For example one may choose to eliminate all $\left(x^{-}\right)^{k}$ with $k \neq 0,1$ (because the constraint is quadratic in $x^{-}$) in order to get a unique representative within the equivalence class of the expression. Unfortunately this particular expression is typically not the most economical one. However such a representative can be used for the purpose to show that a certain expression is identically zero (or that two expressions are equivalent).

In this appendix we would like to present various identities involving the $x^{ \pm}$which were used in the paper or may prove useful otherwise.

One set of $x^{ \pm}$:

$$
\begin{aligned}
& \begin{aligned}
& u=q^{-1} u\left(x^{+}\right)-\frac{\mathrm{i}}{2 g}=q u\left(x^{-}\right)+\frac{\mathrm{i}}{2 g}=\frac{\mathrm{i}}{g} \frac{q^{\mp 1} x^{ \pm} s\left(x^{ \pm}\right)-\frac{1}{2}\left(q+q^{-1}\right)}{q-q^{-1}} \\
& q^{-1} x^{+} s\left(x^{+}\right)=q x^{-} s\left(x^{-}\right)=-\mathrm{i} g\left(q-q^{-1}\right) u+\frac{1}{2}\left(q+q^{-1}\right) \\
&=-\mathrm{i} g q^{-1}\left(q-q^{-1}\right) u\left(x^{+}\right)+q^{-1} \\
&=-\mathrm{i} g q\left(q-q^{-1}\right) u\left(x^{-}\right)+q \\
&=\frac{q^{C} U-q^{-C} U^{-1}}{q^{-C} U-q^{C} U^{-1}} \\
& \frac{x^{+}-s\left(x^{-}\right)}{x^{-}-s\left(x^{+}\right)}=\frac{\left(x^{+}\right)^{2}+1}{\left(x^{-}\right)^{2}+1} \frac{x^{-}+s\left(x^{-}\right)}{x^{+}+s\left(x^{+}\right)}=q^{2 C} U^{2} \\
& q / x^{+}-q^{-1} / x^{-}-\mathrm{i} g^{-1}=-\frac{x^{+}-x^{-}}{q^{-1} x^{+} s\left(x^{+}\right)}=-\frac{x^{+}-x^{-}}{q x^{-} s\left(x^{-}\right)}
\end{aligned}
\end{aligned}
$$


$q^{C} U \frac{\left(x^{-}\right)^{2}+1}{x^{-}+s\left(x^{-}\right)}=q^{-C} U^{-1} \frac{\left(x^{+}\right)^{2}+1}{x^{+}+s\left(x^{+}\right)}=\frac{x^{+}-x^{-}}{q^{C} U-q^{-C} U^{-1}}$.

One $y$ :

$$
\begin{aligned}
& \frac{y^{2}+1}{y+s(y)} \frac{s(y)^{2}+1}{y+s(y)}=1-\left(q-q^{-1}\right)^{2} g^{2} \\
& u(y)=\frac{\mathrm{i}}{g} \frac{y s(y)-1}{q-q^{-1}} .
\end{aligned}
$$

One set of $x^{ \pm}$and $a y$ :

$$
\begin{aligned}
& \frac{x^{+}-s(y)}{x^{-}-s(y)} \frac{s\left(x^{-}\right)-y}{s\left(x^{+}\right)-y}=q^{2 C} U^{2} \\
& \frac{x^{+}-y}{x^{-}-y} \frac{s\left(x^{+}\right)-y}{s\left(x^{-}\right)-y}=\frac{u\left(x^{+}\right)-u(y)}{u\left(x^{-}\right)-u(y)} .
\end{aligned}
$$

Two sets of $x^{ \pm}$:

$$
\begin{aligned}
& \frac{x_{1}^{+}-x_{2}^{+}}{x_{1}^{-}-x_{2}^{-}} \frac{s\left(x_{1}^{-}\right)-s\left(x_{2}^{-}\right)}{s\left(x_{1}^{+}\right)-s\left(x_{2}^{+}\right)}=\frac{x_{1}^{+}-s\left(x_{2}^{-}\right)}{x_{1}^{-}-s\left(x_{2}^{+}\right)} \frac{s\left(x_{1}^{-}\right)-x_{2}^{+}}{s\left(x_{1}^{+}\right)-x_{2}^{-}}=q^{2 C_{1}+2 C_{2}} U_{1}^{2} U_{2}^{2} \\
& \frac{x_{1}^{+}-x_{2}^{+}}{x_{1}^{-}-x_{2}^{-}} \frac{x_{1}^{+}-s\left(x_{2}^{+}\right)}{x_{1}^{-}-s\left(x_{2}^{-}\right)}=q^{2 C_{1}+2} U_{1}^{2} \\
& \frac{x_{1}^{+}-x_{2}^{-}}{x_{1}^{-}-x_{2}^{+}} \frac{x_{1}^{+}-s\left(x_{2}^{-}\right)}{x_{1}^{-}-s\left(x_{2}^{+}\right)}=q^{2 C_{1}} U_{1}^{2} \frac{u\left(x_{1}^{+}\right)-u\left(x_{2}^{-}\right)}{u\left(x_{1}^{-}\right)-u\left(x_{2}^{+}\right)} .
\end{aligned}
$$

\section{Appendix B. Braiding from scattering problem}

An equivalent approach to derive and incorporate the braiding factors for the coproduct (2.45) is based on using an $S$-matrix which acts as a permutation operator and commutes with the coproduct $[\Delta(\mathfrak{J}), \mathcal{S}]=0$ for each generator $\mathfrak{J} \in \mathrm{U}_{q}(\mathfrak{h})$. This relation is equivalent to cocommutativity (2.49) upon identifying the $S$-matrix with the $R$-matrix as usual $\mathcal{S}=\mathcal{P} \mathcal{R}$.

What we want to stress here is the fact that for the quantum-deformed case the introduction of the braiding in the coproduct is necessary to allow for a quasi-cocommutative Hopf algebra. If one would only take the quantum-deformed algebra with the coproduct (2.37) there cannot be any $R$-matrix which transforms $\Delta$ to $\Delta_{\text {op }}$ for the central elements. We will identify the additional braiding element with the central charges in such way that the coproduct will indeed become cocommutative on the center.

In particular, let us consider two short modules with central charges $\left\langle C_{1}, P_{1}, K_{1}\right\rangle$ and $\left\langle C_{2}, P_{2}, K_{2}\right\rangle$. For the coproduct (2.37) we have

$$
\Delta(\mathfrak{P})=\mathfrak{P} \otimes 1+q^{2 \mathfrak{C}} \otimes \mathfrak{P}
$$

but

$$
\Delta_{\text {op }}(\mathfrak{P})=1 \otimes \mathfrak{P}+\mathfrak{P} \otimes q^{2 \mathfrak{C}}
$$

We need to change the coproduct by introducing an additional braiding factor to make it quasi-cocommutative. The importance of having a quasi-cocommutative Hopf algebra lies in 


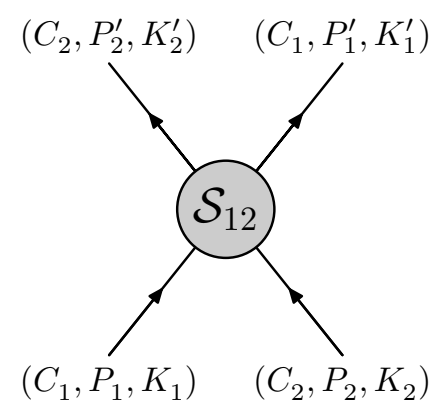

Figure 4. Scattering process and transformation of central charges.

the fact that they have an $R$-matrix which not only intertwines the modules, but also satisfies the quasi-triangularity condition from which the Yang-Baxter equation follows.

Pairwise scattering. Now we will consider the representation structure when the $S$-matrix acts on chains of multiplets. First we consider the scattering matrix of two short multiplets (see figure 4)

$$
\mathcal{S}_{12}:\left\langle\vec{C}_{1}\right\rangle \otimes\left\langle\vec{C}_{2}\right\rangle \longrightarrow\left\langle\vec{C}_{2}^{\prime}\right\rangle \otimes\left\langle\vec{C}_{1}^{\prime}\right\rangle
$$

For each generator $\mathfrak{J} \in \mathrm{U}_{q}(\mathfrak{h})$ we want the $S$-matrix to be invariant

$$
[\Delta(\mathfrak{J}), \mathcal{S}]=0 .
$$

In particular, this relation must hold for the central charges.

An obvious way to conserve the total charge $C_{1}+C_{2}$ is to demand that the individual charges are merely interchanged

$$
C_{1}^{\prime}=C_{1}, \quad C_{2}^{\prime}=C_{2} .
$$

Taking the coproduct (2.37) of the other central charges $\mathfrak{P}, \mathfrak{K}$ and evaluating them before and after scattering we have

$$
\begin{aligned}
& P_{1}+P_{2} q^{2 C_{1}}=P_{2}^{\prime}+P_{1}^{\prime} q^{2 C_{2}}, \\
& K_{1} q^{-2 C_{2}}+K_{2}=K_{2}^{\prime} q^{-2 C_{1}}+K_{1}^{\prime} .
\end{aligned}
$$

Combining the short multiplet constraint (2.29) with (B.5) we have the following equations to be solved:

$$
P_{1} K_{1}=P_{1}^{\prime} K_{1}^{\prime}, \quad P_{2} K_{2}=P_{2}^{\prime} K_{2}^{\prime} .
$$

The substitution

$$
\begin{array}{ll}
P_{1}=1-q^{2 C_{1}} U_{1}, & K_{1}=q^{-2 C_{1}}-U_{1}^{-1}, \\
P_{2}=\left(1-q^{2 C_{2}} U_{2}\right) U_{1}, & K_{2}=\left(q^{-2 C_{2}}-U_{2}^{-1}\right) U_{1}^{-1}
\end{array}
$$

simplifies the calculations dramatically and we obtain ${ }^{13}$

$$
\begin{array}{ll}
P_{1}^{\prime}=\left(1-q^{2 C_{1}} U_{1}\right) U_{2}, & K_{1}^{\prime}=\left(q^{-2 C_{1}}-U_{1}^{-1}\right) U_{2}^{-1}, \\
P_{2}^{\prime}=1-q^{2 C_{2}} U_{2}, & K_{2}^{\prime}=q^{-2 C_{2}}-U_{2}^{-1} .
\end{array}
$$

Factorized scattering. A basic requirement for a factorized $K$-particle $S$-matrix $\mathcal{S}_{\pi}$ for any permutation $\pi \in S_{K}$ is that it forms a representation of the permutation group $S_{K}$, i.e.

13 There are two roots for the second-order equation-one of them corresponds to trivial scattering and we did not mention it here. 
Table 6. The fundamental $S$-matrix of $\mathrm{U}_{q}(\mathfrak{h})$.

$\mathcal{S}\left|\phi_{1}^{1} \phi_{2}^{1}\right\rangle=A_{12}\left|\phi_{2}^{1} \phi_{1}^{1}\right\rangle$
$\mathcal{S}\left|\phi_{1}^{1} \phi_{2}^{2}\right\rangle=\frac{q A_{12}+q^{-1} B_{12}}{q+q^{-1}}\left|\phi_{2}^{1} \phi_{1}^{2}\right\rangle+\frac{A_{12}-B_{12}}{q+q^{-1}}\left|\phi_{2}^{2} \phi_{1}^{1}\right\rangle+\frac{q^{-1} C_{12}}{q+q^{-1}}\left|\psi_{2}^{1} \psi_{1}^{2}\right\rangle-\frac{C_{12}}{q+q^{-1}}\left|\psi_{2}^{2} \psi_{1}^{1}\right\rangle$
$\mathcal{S}\left|\phi_{1}^{2} \phi_{2}^{1}\right\rangle=\frac{A_{12}-B_{12}}{q+q^{-1}}\left|\phi_{2}^{1} \phi_{1}^{2}\right\rangle+\frac{q^{-1} A_{12}+B_{12}}{q+q^{-1}}\left|\phi_{2}^{2} \phi_{1}^{1}\right\rangle-\frac{C_{12}}{q+q^{-1}}\left|\psi_{2}^{1} \psi_{1}^{2}\right\rangle+\frac{q C_{12}}{q+q^{-1}}\left|\psi_{2}^{2} \psi_{1}^{1}\right\rangle$
$\mathcal{S}\left|\phi_{1}^{2} \phi_{2}^{2}\right\rangle=A_{12}\left|\phi_{2}^{2} \phi_{1}^{2}\right\rangle$
$\mathcal{S}\left|\psi_{1}^{1} \psi_{2}^{1}\right\rangle=D_{12}\left|\psi_{2}^{1} \psi_{1}^{1}\right\rangle$
$\mathcal{S}\left|\psi_{1}^{1} \psi_{2}^{2}\right\rangle=\frac{q D_{12} q^{-1} E_{12}}{q+q^{-1}}\left|\psi_{2}^{1} \psi_{1}^{2}\right\rangle+\frac{D_{12}-E_{12}}{q+q^{-1}}\left|\psi_{2}^{2} \psi_{1}^{1}\right\rangle+\frac{q^{-1} F_{12}}{q+q^{-1}}\left|\phi_{2}^{1} \phi_{1}^{2}\right\rangle-\frac{F_{12}}{q+q^{-1}}\left|\phi_{2}^{2} \phi_{1}^{1}\right\rangle$
$\mathcal{S}\left|\psi_{1}^{2} \psi_{2}^{1}\right\rangle=\frac{D_{12}-E_{12}}{q+q^{-1}}\left|\psi_{2}^{1} \psi_{1}^{2}\right\rangle+\frac{q^{-1} D_{12}+q E_{12}}{q+q^{-1}}\left|\psi_{2}^{2} \psi_{1}^{1}\right\rangle-\frac{F_{12}}{q+q^{-1}}\left|\phi_{2}^{1} \phi_{1}^{2}\right\rangle+\frac{q F_{12}}{q+q^{-1}}\left|\phi_{2}^{2} \phi_{1}^{1}\right\rangle$
$\mathcal{S}\left|\psi_{1}^{2} \psi_{2}^{2}\right\rangle=D_{12}\left|\psi_{2}^{2} \psi_{1}^{2}\right\rangle$
$\mathcal{S}\left|\phi_{1}^{a} \psi_{2}^{\beta}\right\rangle=G_{12}\left|\psi_{2}^{\beta} \phi_{1}^{a}\right\rangle+H_{12}\left|\phi_{2}^{a} \psi_{1}^{\beta}\right\rangle$
$\mathcal{S}\left|\psi_{1}^{\alpha} \phi_{2}^{b}\right\rangle=K_{12}\left|\psi_{2}^{\alpha} \phi_{1}^{b}\right\rangle+L_{12}\left|\phi_{2}^{b} \psi_{1}^{\alpha}\right\rangle$

$\mathcal{S}_{\pi} \mathcal{S}_{\pi^{\prime}}=\mathcal{S}_{\pi \pi^{\prime}}$. It imposes certain relations on $P_{i}$ and $K_{i}$. We consider the permutation that interchanges three modules as follows:

$$
\mathcal{S}_{\pi}:\left\langle\vec{C}_{1}\right\rangle \otimes\left\langle\vec{C}_{2}\right\rangle \otimes\left\langle\vec{C}_{3}\right\rangle \mapsto\left\langle\vec{C}_{3}^{\prime}\right\rangle \otimes\left\langle\vec{C}_{1}^{\prime}\right\rangle \otimes\left\langle\vec{C}_{2}^{\prime}\right\rangle
$$

This process can be represented in two different ways. The factorized scatterings $\left(23 \rightarrow 3^{\prime} 2^{\prime}\right.$ and $1^{\prime} 3^{\prime} \rightarrow 3^{\prime \prime} 1^{\prime \prime}$ ) lead to the following relations:

$$
P_{3}^{\prime}=P_{2}+P_{3} q^{2 C_{2}}-P_{2}^{\prime} q^{2 C_{3}}, \quad P_{1}^{\prime}=P_{1}
$$

for the process $23 \rightarrow 3^{\prime} 2^{\prime}$ and

$$
\begin{aligned}
P_{3}^{\prime \prime} & =P_{1}^{\prime}+P_{3}^{\prime} q^{2 C_{1}}-P_{1}^{\prime \prime} q^{2 C_{3}} \\
& =\left(P_{1}+P_{2} q^{2 C_{1}}\right)-\left(P_{1}^{\prime}+P_{2}^{\prime} q^{2 C_{1}}\right) q^{2 C_{3}}+P_{3} q^{2 C_{1}+2 C_{2}}, \\
P_{2}^{\prime \prime} & =P_{2}^{\prime}
\end{aligned}
$$

for the process $1^{\prime} 3^{\prime} \rightarrow 3^{\prime \prime} 1^{\prime \prime}$.

Fusion. Furthermore, the overall process can be represented as a pairwise scattering of composite multiplet $\left\langle\vec{C}_{12}\right\rangle$ with $\left\langle\vec{C}_{3}\right\rangle$. The former multiplet is long but for particular values of $\vec{C}_{1}$ and $\vec{C}_{2}$ it splits into two short multiplets. The central charges $P_{1,2,3}^{\prime}$ and $K_{1,2,3}^{\prime}$ become related by the following constraints:

$$
\begin{aligned}
& P_{1}+P_{2} q^{2 C_{1}}+P_{3} q^{2 C_{1}+2 C_{2}}=P_{3}^{\prime}+P_{1}^{\prime} q^{2 C_{3}}+P_{2}^{\prime} q^{2 C_{1}+2 C_{3}}, \\
& K_{1} q^{-2 C_{2}-2 C_{3}}+K_{2} q^{-2 C_{3}}+K_{3}=K_{3}^{\prime} q^{-2 C_{1}-2 C_{2}}+K_{1}^{\prime} q^{-2 C_{2}}+K_{2}^{\prime} .
\end{aligned}
$$

Results (B.12) and solution of (B.13) should correspond and it constrains the form of central charges. An educated guess consists in choosing them in the following way:

$$
\begin{aligned}
& P_{k}=g \alpha\left(1-q^{2 C_{k}} U_{k}^{2}\right) \prod_{j=1}^{k-1} U_{j}^{2}, \\
& K_{k}=\frac{g}{\alpha}\left(q^{-2 C_{k}}-U_{k}^{-2}\right) \prod_{j=1}^{k-1} U_{j}^{-2} .
\end{aligned}
$$

which completely agrees with (2.52). 
The following remark is noteworthy here. We have derived the dependence of the central charges $P$ and $K$ on the braiding factors $U$ by demanding the condition that scattering with a pair of particles can be treated as a successive scattering with one particle and then with another one. Alternatively we can treat $U$ as a function of $P$ and $K$ and put $U U^{-1}=1$ as a constraint (which is for sure satisfied in (B.8) automatically). If this constraint is not satisfied then the Hopf algebra for $\mathfrak{h}=\mathfrak{s u}(2 \mid 2) \ltimes \mathbb{R}^{2}$ cannot be quasi-triangular. We can drop one central charge (say $K$ and thus have $\mathfrak{s u}(2 \mid 2) \ltimes \mathbb{R}^{1}$ algebra); then this relation does not constrain us any longer and the Hopf algebra based on $\mathfrak{s u}(2 \mid 2) \ltimes \mathbb{R}^{1}$ is quasi-triangular.

Fundamental $S$-Matrix. We write the explicit form of the fundamental $S$-matrix $\mathcal{S}=\mathcal{P} \mathcal{R}$ in table 6. Its coefficients are the same as for the fundamental $R$-matrix given in table 2 .

\section{References}

[1] Drinfeld V G 1988 Quantum groups J. Math. Sci. 41898

Jimbo M 1986 Quantum r Matrix for the Generalized Toda System Commun. Math. Phys. 102537

[2] Essler F H L, Frahm H, Göhmann F, Klümper A and Korepin V E 2005 The One-Dimensional Hubbard Model (Cambridge, UK: Cambridge University Press) p 690

[3] Lieb E H and Wu F Y 1968 Absence of Mott transition in an exact solution of the short-range, one-band model in one dimension Phys. Rev. Lett. 201445

[4] Shastry B S 1986 Exact integrability of the one-dimensional Hubbard model Phys. Rev. Lett. 562453

[5] Ramos P B and Martins M J 1997 Algebraic Bethe ansatz approach for the one-dimensional Hubbard model J. Phys. A: Math. Gen. 30 L195 (Preprint hep-th/9605141)

[6] Martins M J and Ramos P B 1998 The quantum inverse scattering method for Hubbard-like models Nucl. Phys. B 522413 (Preprint solv-int/9712014)

[7] Lieb E H 1989 Two theorems on the Hubbard model Phys. Rev. Lett. 621201

Yang C N $1989 \eta$ Pairing and off-diagonal long-range order in a Hubbard model Phys. Rev. Lett. 632144

[8] Uglov D B and Korepin V E 1994 The Yangian symmetry of the Hubbard model Phys. Lett. A 190238 (Preprint hep-th/9310158)

[9] Minahan J A and Zarembo K The Bethe-ansatz for $\mathcal{N}=4$ super Yang-Mills J. High Energy Phys. JHEP03(2003)013 (Preprint hep-th/0212208)

Beisert N, Kristjansen C and Staudacher M 2003 The dilatation operator of $\mathcal{N}=4$ conformal super Yang-Mills theory Nucl. Phys. B 664131 (Preprint hep-th/0303060)

Bena I, Polchinski J and Roiban R 2004 Hidden symmetries of the $\mathrm{AdS}_{5} \times S^{5}$ superstring Phys. Rev. D 69046002 (Preprint hep-th/0305116)

Beisert N and Staudacher M 2003 The $\mathcal{N}=4$ SYM integrable super spin chain Nucl. Phys. B 670439 (Preprint hep-th/0307042)

[10] Beisert N 2004 The dilatation operator of $\mathcal{N}=4$ super Yang-Mills theory and integrability Phys. Rep. 4051 (Preprint hep-th/0407277)

[11] Plefka J 2005 Spinning strings and integrable spin chains in the AdS/CFT correspondence Liv. Rev. Rel. 89 (Preprint hep-th/0507136)

[12] Staudacher M 2005 The factorized S-matrix of CFT/AdS J. High Energy Phys. JHEP05(2005)054 (Preprint hep-th/0412188)

[13] Beisert N 2008 The su(2/2) dynamic S-matrix Adv. Theor. Math. Phys. (Preprint hep-th/0511082) at press

[14] Frolov S, Plefka J and Zamaklar M 2006 The $\mathrm{AdS}_{5} \times S^{5}$ superstring in light-cone gauge and its Bethe equations J. Phys. A: Math. Gen. 3913037 (Preprint hep-th/0603008)

[15] Arutyunov G, Frolov S and Zamaklar M 2007 The Zamolodchikov-Faddeev algebra for AdS $_{5} \times S^{5}$ superstring J. High Energy Phys. JHEP04(2007)002 (Preprint hep-th/0612229)

[16] Staudacher M 2005 private communication

[17] Beisert N 2007 The analytic Bethe ansatz for a chain with centrally extended su(2/2) symmetry J. Stat. Mech. 07 P01017 (Preprint nlin.SI/0610017)

[18] Beisert N and Staudacher M 2005 Long-Range PSU(2,2/4) Bethe ansatze for Gauge theory and strings Nucl. Phys. B 7271 (Preprint hep-th/0504190)

[19] Arutyunov G, Frolov S, Plefka J and Zamaklar M 2007 The off-shell symmetry algebra of the light-cone $\mathrm{AdS}_{5} \times S^{5}$ superstring J. Phys. A: Math. Theor. 403583 (Preprint hep-th/0609157) 
[20] Gomez C and Hernández R 2006 The magnon kinematics of the AdS/CFT correspondence J. High Energy Phys. JHEP11(2006)021 (Preprint hep-th/0608029)

Plefka J, Spill F and Torrielli A 2006 On the Hopf algebra structure of the AdS/CFT S-matrix Phys. Rev. D 74066008 (Preprint hep-th/0608038)

[21] Beisert N 2007 The S-Matrix of AdS/CFT and Yangian Symmetry PoS Solvay 002 (Preprint 0704.0400)

[22] Matsumoto T, Moriyama S and Torrielli A 2007 A secret symmetry of the AdS/CFT S-matrix J. High Energy Phys. JHEP09(2007)099 (Preprint 0708.1285)

[23] Torrielli A 2007 Classical R-matrix of the su(2/2) SYM spin-chain Phys. Rev. D 75105020 (Preprint hep-th/0701281)

[24] Beisert N and Spill F 2008 The classical $R$-matrix of AdS/CFT and its Lie Bialgebra structure Comm. Math. Phys. (Preprint arXiv:0708.1762) at press

[25] Essler F H L, Korepin V E and Schoutens K 1992 New exactly solvable model of strongly correlated electrons motivated by high T(c) superconductivity Phys. Rev. Lett. 682960 (Preprint cond-mat/9209002)

[26] Bracken A J, Gould M D, Links J R and Zhang Y-Z 1995 A new supersymmetric and exactly solvable model of correlated electrons Phys. Rev. Lett. 742768 (Preprint cond-mat/9410026)

Bedürftig G and Frahm H 1995 Thermodynamics of an integrable model for electrons with correlated hopping J. Phys. A: Math. Gen. 284453 (Preprint cond-mat/9504103)

Ramos P B and Martins M J 1996 One parameter family of an integrable spl(2/1) vertex model: algebraic Bethe ansatz approach and ground state structure Nucl. Phys. B 474678 (Preprint hep-th/9604072)

Pfannmüller M P and Frahm H 1996 Algebraic Bethe ansatz for gl(2,1) invariant 36-vertex models Nucl. Phys. B 479575 (Preprint cond-mat/9604082)

[27] Maassarani Z 1995 U osp(2,2) lattice models J. Phys. A: Math. Gen. 281305 (Preprint hep-th/9407032)

Bariev R Z, Klümper A and Zittartz J 1995 A new integrable two-parameter model of strongly correlated electrons in one dimension Europhys. Lett. 3285 (Preprint cond-mat/9504114)

Gould M D, Hibberd K E, Links J R and Zhang Y-Z 1996 Integrable electron model with correlated hopping and quantum supersymmetry Phys. Lett. A 212156 (Preprint cond-mat/9506119)

[28] Maassarani Z 1998 The su(n) Hubbard model Phys. Lett. A 239187 (Preprint cond-mat/9709252)

Martins M J 1998 On the integrability of the SU(N) Hubbard model Phys. Lett. A 247218 (Preprint cond-mat/9710049)

Maassarani Z 1998 Exact integrability of the su(n) Hubbard model Mod. Phys. Lett. B 1251 (Preprint cond-mat/9710083)

[29] Maassarani Z 1999 Multiplicity A $m$ Models Eur. Phys. J. B 7627 (Preprint solv-int/9805009)

[30] Gould M D, Links J R, Zhang Y-Z and Tsohantjis I 1997 Twisted quantum affine superalgebra $\mathrm{U}_{q}\left[\mathrm{sl}(2 / 2)^{(2)}\right]$, $\mathrm{U}_{q}[\operatorname{osp}(2 / 2)]$ invariant $R$-matrices and a new integrable electronic model J. Phys. A: Math. Gen. 304313 (Preprint cond-mat/9611014)

Martins M J and Ramos P B 1997 Solution of a supersymmetric model of correlated electrons Phys. Rev. B 566376 (Preprint hep-th/9704152)

Montorsi A 1998 Two-parameter extended Hubbard Hamiltonian with gl(2/1) supersymmetry Eur. Phys. J. B 5419

Foerster A, Hibberd K E, Links J R and Roditi I 2001 Quantum spin ladder systems associated with su(2/2) J. Math. Phys. 34 L25 (Preprint cond-mat/0010035)

Bracken A J, Ge X-Y, Gould M D, Links J R and Zhou H-Q 2001 Algebraic Bethe ansatz for integrable extended Hubbard models arising from supersymmetric group solutions J. Phys. A: Math. Gen. 344459 (Preprint cond-mat/0105256)

Göhmann F 2002 Algebraic Bethe ansatz for the gl(1/2) generalized model and Lieb-Wu equations Nucl. Phys. B 620501 (Preprint cond-mat/0108486)

[31] Schlottmann P 1987 Integrable narrow-band model with possible relevance to heavy Fermion systems Phys. Rev. B 365177

Lai C K 1974 Lattice gas with nearest-neighbor interaction in one dimension with arbitrary statistics J. Math. Phys. 151675

Sutherland B 1975 Model for a multicomponent quantum system Phys. Rev. B 123795

Essler F H L and Korepin V E 1992 A New solution of the supersymmetric t-J model by means of the quantum inverse scattering method Preprint hep-th/9207007

[32] Bariev R Z 1991 Integrable spin chain with two- and three-particle interactions J. Phys. A: Math. Gen. 24 L549

Bariev R Z 1991 Integrable model of interacting XY chains J. Phys. A: Math. Gen. 24 L919

[33] Bariev R Z 1994 Exact solution of generalized t-j models in one dimension J. Phys. A: Math. Gen. 273381

Bariev R Z, Klümper A, Schadschneider A and Zittartz J 1995 Exact solution of a one-dimensional fermion model with interchain tunneling Phys. Rev. B 509676 (Preprint cond-mat/9409084) 
Bariev R Z, Klümper A, Schadschneider A and Zittartz J 1995 A one-dimensional integrable model of fermions with multi-particle hopping J. Phys. A: Math. Gen. 282437

Alcaraz F C and Bariev R Z 1998 New integrable generalization of the one-dimensional t-j model J. Phys. A: Math. Gen. 31 L233 (Preprint cond-mat/9706301)

[34] Alcaraz F C and Bariev R Z 1999 Interpolation between Hubbard and supersymmetric t-j models: two-parameter integrable models of correlated electrons J. Phys. A: Math. Gen. 32 L483 (Preprint cond-mat/9908265)

[35] Kamupingene A H, Ky N A and Palev T D 1989 Finite-dimensional representations of the Lie superalgebra $\operatorname{gl}(2 / 2)$ in a $g l(2) \oplus g l(2)$ basis. I. Typical representations J. Math. Phys. 30553

Palev T D and Soilova N I 1990 Finite-dimensional representations of the Lie superalgebra gl(2/2) in a $g l(2) \oplus g l(2)$ basis. II. Nontypical representations J. Math. Phys. 31953

[36] Zhang Y-Z and Gould M D 2005 A unified and complete construction of all finite-dimensional irreducible representations of gl(2/2) J. Math. Phys. 46013505 (Preprint math.qa/0405043)

[37] Ky N A 1994 Finite-dimensional representations of the quantum superalgebra $\mathrm{U}_{q}(\mathrm{gl}(2 / 2))$ : 1. Typical representations at generic $q$ J. Math. Phys. 352583 (Preprint hep-th/9305183)

Ky N A and Stoilova N I 1995 Finite-dimensional representations of the quantum superalgebra $\mathrm{U}_{q}(\mathrm{gl}(2 / 2)) .2$. Nontypical representations at generic q J. Math. Phys. 365979 (Preprint hep-th/9411098)

[38] Arutyunov G and Frolov S 2007 On String S-matrix, Bound States and TBA J. High Energy Phys. JHEP12(2007)024 (Preprint arXiv:0710.1568)

[39] Janik R A 2006 The $\mathrm{AdS}_{5} \times S^{5}$ superstring world sheet $S$-matrix and crossing symmetry Phys. Rev. D 73086006 (Preprint hep-th/0603038)

[40] Chen H-Y, Dorey N and Okamura K 2007 The asymptotic spectrum of $\mathcal{N}=4$ super Yang-Mills spin chain J. High Energy Phys. JHEP03(2007)005 (Preprint hep-th/0610295)

[41] Beisert N 2007 On the scattering phase for $\mathrm{AdS}_{5} \times S^{5}$ strings Mod. Phys. Lett. A 22415 (Preprint hep-th/0606214)

Beisert N, Hernández R and López E 2006 A Crossing-Symmetric Phase for $\mathrm{AdS}_{5} \times S^{5}$ Strings J. High Energy Phys. JHEP11(2006)070 (Preprint hep-th/0609044)

[42] Yang C N 1967 Some exact results for the many body problems in one dimension with repulsive delta function interaction Phys. Rev. Lett. 191312

[43] Martins M J and Melo C S 2007 The Bethe ansatz approach for factorizable centrally extended $S$-matrices Nucl. Phys. B 785246 (Preprint hep-th/0703086)

[44] Woynarovich F 1983 Low-energy excited states in a Hubbard chain with on-site attraction J. Phys. C: Solid State Phys. 166593

[45] Reshetikhin N 1990 Multiparameter quantum groups and twisted quasitriangular Hopf algebras Lett. Math. Phys. 20331

[46] Reshetikhin N Y and Wiegmann P B 1987 Towards the classification of completely integrable quantum field theories Phys. Lett. B 189125

Ribeiro G A P and Martins M J 2006 Algebraic Bethe Ansatz for an integrable $\mathrm{U}_{q}(\mathrm{sl}(n / m))$ vertex model with mixed representations Nucl. Phys. B 738391 (Preprint nlin.SI/0512035)

[47] Leigh R G and Strassler M J 1995 Exactly marginal operators and duality in four-dimensional $\mathcal{N}=1$ supersymmetric gauge theory Nucl. Phys. B 44795 (Preprint hep-th/9503121)

Lunin O and Maldacena J 2005 Deforming field theories with U(1) $\times$ U(1) global symmetry and their gravity duals J. High Energy Phys. JHEP05(2005)033 (Preprint hep-th/0502086)

[48] Roiban R 2004 On spin chains and field theories J. High Energy Phys. JHEP09(2004)023 (Preprint hep-th/0312218)

[49] Berenstein D and Cherkis S A 2004 Deformations of $\mathcal{N}=4$ SYM and integrable spin-chain models $N u c l$. Phys. B 70249 (Preprint hep-th/0405215)

[50] Frolov S A, Roiban R and Tseytlin A A 2005 Gauge-string duality for superconformal deformations of $\mathcal{N}=4$ super Yang-Mills theory J. High Energy Phys. JHEP07(2005)045 (Preprint hep-th/0503192)

Månsson T 2007 The Leigh-Strassler deformation and the quest for integrability J. High Energy Phys. JHEP06(2007)010 (Preprint hep-th/0703150) 